\title{
Trading Frames:
}

\section{Interface Operations and Social Exchanges in Video Games}

by

Adam Benn

A thesis submitted to the Faculty of Graduate and Postdoctoral Affairs in partial

fulfillment of the requirements for the degree of

Doctor of Philosophy

in

English Language and Literature

Carleton University

Ottawa, Ontario

(C) 2017, Adam Benn 


\begin{abstract}
This dissertation examines trade affordances across three different video games and one novel: The Realm Online (1995), World of Warcraft (2004), Counter-Strike: Global Offensive (2012), and Neal Stephenson's Reamde (2011). By trade affordances, I refer to those interfaces which facilitate the transition of digital items from one player to another. While the study of online economies has an established history, the broader social impact of trade affordances remains largely unexplored despite their ubiquity. I align these aforementioned video games with an increasing automation of trade practices within contemporary multi-user online games, as well as the growing relationship between online and offline economies. In order to demonstrate these connections, I provide a summary of early trade practices collected through blogs, playthroughs, developer notes, patches, and other ethnographic sources such as interviews and forum posts. After describing these trade practices, I survey key economic, ethical, political, and social theories relevant to the act of trading and consuming in online spaces. My critique is influenced by autonomist Marxist theory regarding the automation of work and the cycles of struggle central the relationship between labour and capital. This positions my dissertation in relation to other game studies scholars who have assessed the relationship between play and labour in video games, such as Nick Dyer-Witherford, Alexander Galloway, and McKenzie Wark. I contend that trade in online games is an increasingly capitalized act reflective of conditions of capital outside the games. In order to demonstrate this phenomenon, I provide close-readings of the previously mentioned video games and novel, as well as two single-player games that directly critique the relationship between trade, capital, and play.
\end{abstract}




\section{Acknowledgements}

I would like to thank my supervisor, Dr. Brian Greenspan, for his tireless effort in supervising me throughout my degree. His input has been invaluable, and I can only hope in vain to replicate his work effort and expertise. In addition to guiding this dissertation, Dr. Greenspan's supervision brought me to conferences, workshops, and design seminars I would never have had the opportunity to visit otherwise. I would also like to thank Dr. Sarah Brouillette for her support. Her insight has been crucial to me finishing this work. She read drafts and provided commentary long before she needed to, and went above and beyond her role as a member of my supervisory committee. I wish to thank Dr. Travis DeCook, as well, for supervising my comprehensive exams. I am also grateful to Dr. Bart Simon and Dr. Shawn Graham for agreeing to act as external examiners at my defense.

I am thankful to my colleagues, as well, who have helped me throughout my degree. Chris Reid, Bradley Weiers, Ron Mackenzie, David Wintle, Ryan Prager, Eve Robinson, Jan Michael, Cameron Ruether, Steve Mcleod, Adrien Robertson, and Alexander Grammatikos all provided, at some time or another, much needed technical, intellectual, or emotional support. I am certainly missing names and I apologize for that. You know who you are, and I am thankful for your help.

I am eternally grateful to my mother, Belinda, who worked harder than anyone to ensure I could finish this degree. 


\section{Table of Contents}

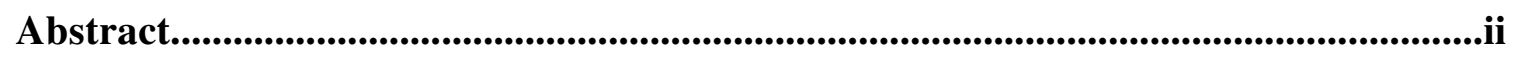

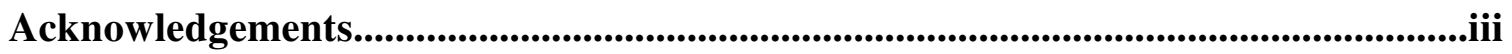

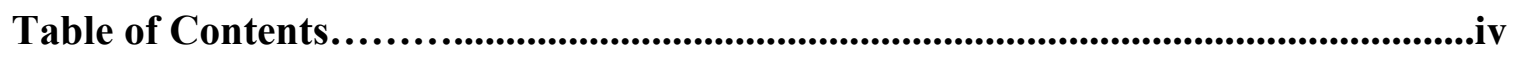

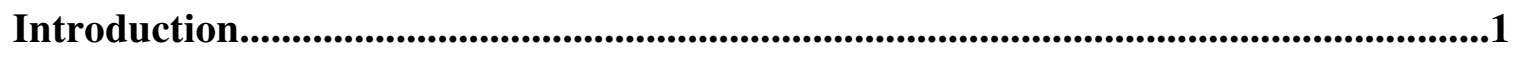

The Realm Online and Early MMOs.............................................................6

World of Warcraft and Advancements in Trade Affordances............................14

Reamde and the Growth of Digital Trade......................................................19

The Steam Marketplace's Organizational Structure, Esports, and Skin

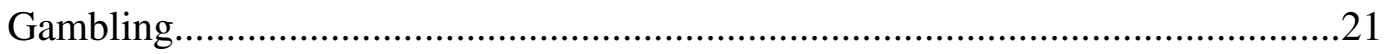

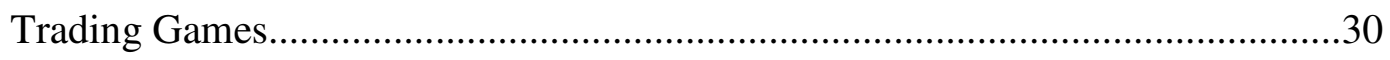

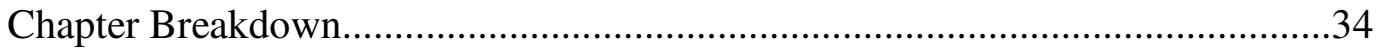

Chapter 1: A Review Concerning Economics, Interfaces, Players, and Ethics..........36

1.1 Demographics to Set the Stage of Exchange...................................36

1.2 Economic Perspectives, Old and New....................................46

1.2.1 Classical Economics and Trade Motivations................................47

1.2.2 Trade Affordances as Institutional Forms..................................53

1.3 The Influence of Interface and the Role of the Player.................................65

1.3.1 Interface as Rhetoric. ..........................................................65

1.3.2 Video Game Mechanics and Interfaces......................................69

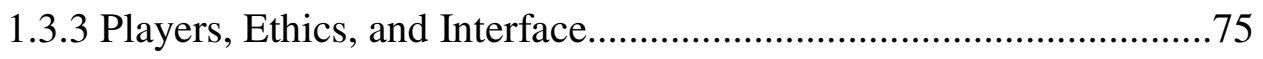

1.4 Marxisms and Historical Materialisms...................................................91 
1.4.1 Technology, Labour, and Production.

Chapter 2: Interpersonal Exchange in The Realm and World of Warcraft..............109

2.1 The Realm and the Socialised Exchange................................................112

2.2 The Material, Social, and Cultural Implications of the Auction House........120

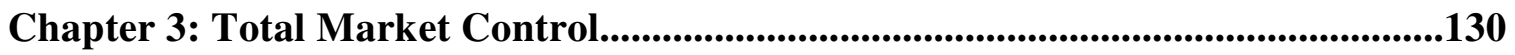

3.1 Reamde Predicts The Future.................................................................131

3.2 Brokering the Steam Market..................................................................139

Chapter 4: The Avant-Garde, the Monetization of Play, and Evil..........................160

4.1 Trucking Along in Papers, Please ........................................................

4.2 Bartering With Evil in Recettear........................................................173

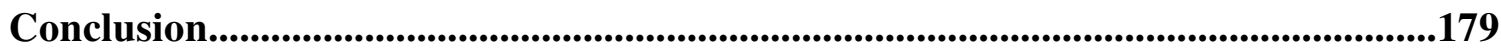

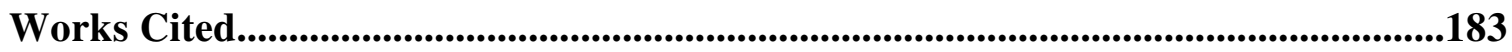




\section{Introduction}

This dissertation examines the cultural, economic, and political impact of three different video game trade affordances. These games or trade interface are separated by almost a decade, and each demonstrates distinct properties which align it historically with broader transformations in virtual trade and consumption. While other games and interfaces enter the discussion, the major objects of study in this dissertation are The Realm Online (1995), World of Warcraft (2004), and the Steam Market. The Steam Market is not itself a game (although users have treated it as one), but a market created to govern various in-game economies. Steam itself is a digital platform for distributing thousands of games from various developers. In order to provide a meaningful and focused discussion of its operations, however, I will address the Steam Market in relation to Counter-Strike: Global Offensive (2012) and the game's associated esports scene. Additionally, I provide close-readings of two single-player games (Papers, Please and Recettear) and one novel (Reamde) that comment critically on the conditions of exchange in video games.

Each object of study marks technical and cultural shifts that are described in relation to their particular trade affordances. Throughout this dissertation I show that advancements in video game technologies have resulted in increasingly automated trade practices. Video game technologies here refers to software, hardware, and networks. For

example, increased internet access and speed, server memory, and computer power are all contributing factors for massively multiplayer online games (MMOs) such as World of Warcraft (WoW) to produce a boisterous and active trading economy. Moreover, auction houses relieved players of having to manually chat and meet in avatar to trade, thereby 
automating elements of trade practises found in early games such as The Realm Online (The Realm). These practices are described in detail in the object description section. The World of Warcraft auction house has evolved since its inception, and its programming has been consistently modified to decrease the need for player-to-player interaction as far as trading. Transcending a single game, the Steam Market is an umbrella institution which enables players to trade items across a variety of games distributed by Steam.

This dissertation does not provide any formal description of what constitutes a video game, but instead relies on describing attributes and characteristics common among video games. As a result, I defer to Bernard Suits' (2005) definition of a game: "playing a game is the voluntary attempt to overcome unnecessary obstacles" (The Grasshopper 55). I always thought there was so much power in this definition, and for a few years I could not identify why I found it so compelling. Part of it is the liberating power of the statement that players voluntarily play. Suits explains further that "to play a game is to attempt to achieve a state of affairs [prelusory goal], using only means permitted by the rules [lusory means], where the rules prohibit use of more efficient in favour of less efficient means [constitutive rules], and where the rules are accepted just because they make possible such activity [lusory attitude]" (48). It is liberating to imagine this state, or enact it by knowingly and actively embodying lusory attitudes, spaces, and rules. The game world Suits describes is a place beyond the toils of everyday life: predefined interpersonal relationships, jobs, family, and other structures that shape the non-gamic moments of human life. In fact, Suits' game worlds might be considered a form of escape rather than a liberating pastime. Of course, lived experiences happen and cultural 
contexts matter even within these game spaces. Nevertheless, pre-digital theorists like Suits, Johan Huizinga, and Roger Caillois imagine games as temporary spaces momentarily distinct from the real world. As Huizinga (2006) puts it, games are "all temporary worlds within the ordinary world, dedicated to the performance of an act apart" ("Nature and Significance of Play" 98). For these theorists, all meaning and value within these spaces is provisional and temporary.

Paramount to Suits' definition of playing a game is efficiency--or, rather, inefficiency. For Suits, games are a waste of time and that can be liberating, escapist, or both. The obstacles and enemies that games present to their players are unnecessary because they are provisional and imagined. The demons and dragons that players battle are separate from their everyday lives, but so too are the rewards for such conquests. Rules, according to Suits, are there to achieve a state where inefficiency is a property of play. He explains that "rules function to prohibit use of the most efficient means" (20). One wonders if there are any games left, then, given the rise of efficiency documented throughout this dissertation.

The other reason I found Suits' definition so compelling is precisely because it feels so antiquated. The first edition of Dungeons \& Dragons (1974), for example, lacked many clear rules for combat situations and item management. ${ }^{1}$ Thirty-three years later, the fifth edition of the game provides hundreds of supplementary materials for players, ranging from miniature figurines and battle mats to place these figurines on, to secondary books of information regarding specialized combat, classes, and materials, all of which are tightly managed and itemized on character sheets and delegated by scripted

1 Corporation 9592, the fictional entity running the MMO in Stephenson's novel Reamde, was inspired by $D \& D$. The protagonist, Richard Forsythe, rolls $D \& D$ dice that were in his pocket to randomly generate the name of his company (35). 
adventures with standardized play periods. Dungeons \& Dragons has become, in effect, an accounting simulator. It is but one nascent example of the growing efficiency and itemization that marks the video gaming experience. Moreover, $D \& D$ 's swords-andsorcery world is a massive influence for many early and contemporary MMOs. As it happens, $D \& D$ 's transformation pales in comparison to developments in digital gaming, particularly with regards to efficiency and itemization.

Unlike the world Suits describes, nowadays play is persistent and productive. Gaming, broadly speaking, is integrated into people's everyday lives. Players may play voluntarily, but in so doing, they involuntarily produce labour for which they see no monetary recompense, in an increasingly automated trade market. Steam, as I note below, collects dividends from exchanges, and as in many other games, players create items simply by playing, thereby driving the overall collective economy. Marxist critics such as Nick Dyer-Witherford, Antonio Hardt, Michael Negri, McKenzie Wark, Alexander Galloway, and Christian Fuchs have written at length about capitalism's reliance on new areas of labour and an associated shift towards a knowledge and entertainment economy. This dissertation will point out on numerous occasions the relationship between divisions of labour, skill sets, and trade which contribute to efficient trade practices that either reveal or encourage economic inequality.

I draw in particular on Nick Dyer-Witherford's (1999) reading of Autonomist Marxism. This dissertation positions the growing automation of trade as part of an ongoing cycle of struggle between a player-base who actively produces value and developers who exploit the economic activity of their users. As Dyer-Witherford "locates the origins of the information society in the conflict between labour and capital," 
this dissertation attempts to similarly identify trade affordances as a site of struggle between players (labour) and developers (capital) (Cybermarx 20). I discuss how trade practices have become increasingly automated, thereby reducing the ability of players to perform various roles and functions within the game, and increasing real money transactions.

Throughout this dissertation, I imagine player agency as operationally, socially, and politically diverse. Player agency, for example, is available in some areas of game play but not in others. Sicart contends that players' reactions to rule changes can be an instance of ethical agency among players (118). However, not all instances of interactions between players, rule structures, games, designers, mechanics, or interfaces allow for agency among players. I contend that there are occasions in which players are either unaware of broader economic or political concerns or uninterested in making changes. A structuralist Marxist philosophy guides my concerns and criticisms with regards to gaming and capitalism throughout this dissertation, and I accept that there are certainly players who are not concerned with the same issues I am. As such, it is important to note that not all players are duped into playing at capitalism, so to speak, and that they do not necessarily see capitalist ideology as problematic a priori. The Auction House, the Steam Market, and other similar arbitrage systems are not necessarily in and of themselves playing at capitalism, however. Instead, trading in games is problematic because of the systems of capital that surround games and interfaces which enable a system that creates value from the act of play. The secondary markets, private propertybased EULAs, and interfaces that enable these markets are the nexus for the critique I make throughout this dissertation. Players have no agency over their involvement in the 
structures of capital that derive value from simply having these markets populated.

However, as I demonstrate, players have made occasional efforts to direct the market's

energies toward their own goals. In essence, players are incapable of existing outside the realm of value creation under capitalism, but are sometimes able to manipulate the structures of capital to benefit themselves. Nevertheless, the markets I write about in this dissertation create value simply by being populated and generating items and information, and in that regard there is no agency possible for players. As I demonstrate, the surrounding structures that enable real money trading are outside of the realm of player agency.

\section{The Realm Online and Early MMOs}

Early MMOs often found themselves mimicking the MUDs that preceded them, both in style of play and in trade practices (if trading was possible at all). First generation 3D MMOs often took on an ahistorical imagination, gesturing toward feudal practices. These games often involved wizards, quests, races of humanoids inspired by Tolkien's fiction, character alignments borrowed from Dungeons and Dragons, fantastical items, currencies, and feudal towns complete with various halls, meaderies, bars, and action houses. Mirridian 59, The Realm Online, Ultimate Online, and various other early MMOs follow this aesthetic design. The game I am using as an object of study in this case is The Realm, although many other early MMOs did not have these interfaces either.

The game world of these early MMOs, much like contemporary games, was a mix of quests undertaken in order to attain a higher level, better items, and a new challenge. 
Players had a variety of skills, classes, and items available to them. Choosing various skills and acquiring particular items allowed for groups of players to compliment a group who had decided to work together (referred to commonly as a guild). By forming a group or guild, players could better navigate a world filled with Non-Player Characters (NPCs) and Player Characters (PCs). Players had various motivations for choosing or seeking out any items, skills, or class (warrior, rogue, or wizard are some generic examples), but the ability to work within a group or achieve a high power level was central to many players.

The reason to attain a high power level in these games is that items dropped by more powerful enemies were scarce commodities within the market. Drops are rewards for defeating NPCs or monsters in a game or completing a quest. Drops are percentage based, with higher power level items programmed to drop at a lower probability. Thus, groups and guilds formed to grind out high level dungeons or enemies in order to increase the amount of high level gear they could acquire. The item scarcity built into these early MMOs remains an integral design property throughout contemporary games today, even in random chance purchases within the Steam market that I describe later. ${ }^{2}$ Of course, this programmed scarcity results in a desire among players to trade items among the community.

Early MMOs lacked much in the way of technical resources or interfaces to

2 While item scarcity is more relevant to this study, scarcity among classes and skills was and is common among MMOs. There are often high-end guilds looking for someone to perform tasks that the majority of players with guild can or prefer not to do. For example, it is common to see groups or guilds looking for a role to fill: a cleric, a tank, a wizard, or whatever skill they are missing. At the competitive level, this is to be expected, as the majority of people wanting to grind levels and high power items will take classes that are self-sufficient as opposed to, say, a support role. Forums and in-game posts are often filled with people seeking a missing piece of the meta-game required to defeat high powered NPCs or quests. Scarcity is central to MMOs in more ways than items and currencies. 
manage this scarcity on a social level. From a technical standpoint, dial-up internet and server maintenance were issues that prevented the continuous and sustained trade available to players today. Maintenance of servers, or even crashes, were common enough to prevent widespread ability to acquire items at a rate as efficient and calculated as contemporary games. Furthermore, some early MMOs lacked any interface to exchange items between players. Players could instead drop the item on the ground (a hold-over technique from MUDs), or click-and-drag an item from their inventory to give it to another player.

The major technical apparatuses provided by these games - The Realm in particular - was designated auction houses. These auction houses required players to show up in person, in real-time, and have a player act as an auctioneer. The auctioneer might hold on to a variety of items from different sellers, take bids, and divide up the profits to the sellers after distributing items to buyers. Other auction houses worked more like a large outdoor market where players would gather and chat within the local area about what they had available, and find a way to make the exchange. Many early MUDs and MMOs did not have graphic user interfaces to facilitate trade cleanly and efficiently until the EverQuest and World of Warcraft era (1999, 2004).

This dissertation specifically focuses on The Realm for three reasons. The first reason is methodological: the game is still (barely) active, and has not updated its user interface regarding trading since its release. As a result, it is possible to depict some of the early trading practices using screenshots, anecdotes, and websites. For example, various user-created websites exist that describe the trading process and provide tips for new players on how to manage it. More relevantly, on the game's $20^{\text {th }}$ anniversary 
(2016), creator Stephen Nichols publicly stated his desire to create a sequel, The Realm Reawakened, and began attempting to crowd source the expenses through GoFundMe. Nichols comments resulted in numerous reviews, photos, forum posts, anecdotes, discussions, and archives being dredged up, while simultaneously sparking newfound interest and commentary on the state of the game. Humorously, some game journalists returned to The Realm to find it populated by only a few dozen players, but in so doing have contextualized the game in relation to other MMOs (AltasObscura and GiantBomb). In any case, this prodigious surplus of information has informed the present research.

Second, The Realm is historically relevant as one of the early attempts at designing and marketing an MMO. Eric Grundhauser (2013) notes that it "might well be the world's oldest still-operating multiplayer online role playing game" ("It's a Mystery" n. pag.). The flagship website is filled with low-resolution images resembling .jpegs copy-and-pasted over a word document, and advertises "dial-up friendly" and "basic system requirements" as some of the game's selling points. Grundhauser describes the game's history:

It was first released in late 1996, by then industry giant, Sierra Games. The game was a very early version of a MMORPG, featuring Dungeons-and-Dragons-style character creation, and an open world where you could meet up with other player characters and quest for loot, hunt monsters, or just chat. Today these features seem unremarkable, but in 1996, when the connectivity and community on the internet was still figuring itself out, The Realm was revolutionary. (par. 2).

The historical significance of the game, then, is one of the reasons I chose it as an object of study. As Grundhauser notes, Sierra On-Line helped set industry standards for video 
game design during the late 1980s and early 1990s. For example, their release of King's Quest V in 1990 won numerous awards and reportedly sold 500,000 copies ("Kings Quest V" n. pag.). While largely forgotten, and abandoned by Sierra after the arrival of EverQuest, the game represents a unique moment in online video game trading history in which items were graphically represented, in-game economies flourished, and player communities navigated these new massively multiplayer territories.

The third reason I chose to write about The Realm is familiarity. Celia Pearce's Communities of Play (2011) provides an example of video game ethnography that influences this research specifically through the author's direct engagement with anecdotal histories and emergent communities. Pearce condones active participation in the culture of study, and explains that the reason for doing so "is more technical than philosophical: you cannot observe a virtual world without being inside it, and in order to be inside it, you have to be 'embodied"' (196). While the history and descriptions I provide come from secondary sources, including games journalism, game historians, amateur archaeologists, and player anecdotes, my interpretation of the game's trading culture is also informed by nearly a decade spent playing the game, largely as a notorious griefer.

The Realm borrows many design elements from MUDs, evidenced by its navigational system and 2D point-and-click environment. In fact, The Realm was originally prototyped as a MUD but Sierra designer Roberta Williams insisted on exploring further possibilities with the game ("RPG Nostalgia" n. pag.). Grundhauser explains that The Realm's game world "is set up in a massive grid of interlocking static screens, and [players] travel between them by clicking on the appropriate side of the 
game window, or on entrances elsewhere on the screen. In essence your character travels from one pixelated tableau to the next" (par 11). Directions in the game are given by direction, location, and number of screens. For example, a common meeting spot located two screens south of the village of Monmouth would be described as "2SMM." Of note, the game contained no Heads-Up-Display (HUD) interface that is common in many video games today. Players could download a map separately or ask directions on one of the chat channels. One method of social interaction was chat channels built into the game as well as a local area chat to enable players within close proximity to speak as if in the same room. This is an influential design process that has its history in MUDs and AOLera games, but has carried over to contemporary MMOs as well.

Despite its antiquated qualities associated with MUDs, The Realm also contained a boisterous economy driven by a peak player-base of approximately $25,000 .^{3}$ While this number pales in comparison to the roughly ten million players one might find on World of Warcraft servers, it represents, within a single game server, a sizable enough population to produce numerous guilds, factions, communities, and, most importantly for this research, items with which to truck and barter.

In The Realm, making an exchange of items can be done in a few ways. There is an entire strategy guide for trading published by a user named SandyClaws (of the eponymous website):

Once you and another person agree on a [trade], you need to go and physically trade the items. This can be dangerous, as you run a risk of losing your item. There are three options for the transfer of items. Either you can hand your [item]

3 Number of players taken from the game's official website, realmserver.com, but corroborated by secondary sites and archived reviews. 
to the other person, and trust him/her to give you the money, or the other person can hand you the money, and trust you to give them the item. Or, as usually happens, one party or the other requests the services of an MP [a middle-person]. ("Trading Guides!")

I will describe these processes below, but notably this guide misses one other methods of making an exchange which I will quickly explain. Apart from giving an item to another player, The Realm's user-interface allowed players to drop items on the ground. Two parties dropping items simultaneously was a common trade practice in early MMOs, and occurred in The Realm as well. ${ }^{4}$ These exchanges happened when two players met in a physical location close enough to verify the item on the ground by hovering their mouse over it, but not close enough to move and pick it up before its owner could. Once the items were verified, players would quickly maneuver their avatar to the item and pick it up. Of course, these trades would be stressful and required a quick, reliable, and stable internet connection, as lag could result in a robbery.

Clicking an item in one's inventory would allow a player to click and drag it to another player's avatar and select "give," transferring the item into its new owner's inventory. There was no method of timing the exchange in order to ensure each party received the proper item. The technical limits of the game did not allow a visual exchange. Instead, a player's avatar simply demonstrated a hand motion indicating they

4 Interestingly, the first Diablo game resulted in the community employing drop-trades despite having trade windows to facilitate trade. There were technical glitches in the game's trading system. Players could put fake items in the place of valuable ones before the trade was confirmed in the window, or quickly remove them before the other player hit accept. The latter of these scams was possible to due to latency issues on servers or personal computers that prevented players from accepting the trade in real-time, seeing instead a delayed image of an item already gone. The former scam was simply a method of abusing a new trading system that would crash or misread certain items. As a result, players would create middleperson service servers via bulletin posts, or meet in a server and drop items and then quickly move across the screen to pick them up while the other player did the same. 
had given something, and then the recipient would have to verify in their inventory GUI that it was the correct item. As a result, giving became a social phenomenon as well as a technical one, as players managed their desire to make profitable or mutually beneficial trades.

One method of exchange was for two parties to meet and debate about who should "give first." Give first is a term that implied the recipient was more trustworthy. Players would often settle on who gave first by asking the global trade chat channel. While other MUDs and some early MMOs used out-of-game message boards and forums, The Realm had various in-game chat channels, one of which was dedicated to trade discussion. This channel would be populated with various community members and avid traders who would either vouch for a player, assure potential traders the player was known to the community, or part of a particular guild (GiantBomb, SandyClaws). Generally, players would take resounding support from trade chat as an acceptable safeguard. In the case that each player was unknown, or had conflicted reports from the community for previous scams, the two parties would search for an alternative.

One of these alternatives included the use of a middle-person (MP). These players operated as trusted human intermediaries for exchanging items between players. Generally, players who engaged in this profession spent a majority of their time in game facilitating trades as opposed to acquiring their own items through typical game play. Guilds would often employ honest and notable MPs to complete trades on behalf of the guild. Many of these noted trade facilitators contained high-power or rare vanity items that were often given to them as gifts by the community. Moreover, SandyClaws notes that volunteer moderators could be contacted by typing /gms and they would often be 
able to recommend or suggest an honest MP, or even operate as one ("Trading Guides!").

Scams occurred in all of these trading methods, which required a certain level of truth among players, but which also evidenced the influence and power of the game's social sphere. Players moved items and the economy functioned without user-interface that governed or safeguarded exchange. Social norms dictated exchange in these early MMOs, thereby linking role-playing and storytelling to trade. Guilds, for example, had bankers or inventory managers who ran the guild's economy and excess items. Additionally, deviant practices such as scamming might result in a player being ostracized from the community, thereby decreasing their ability to not only trade items but to form groups to play the game with. Of note, there were enough villainous players to form their own guilds, creating a sort of rivalry among honest folks and rogues (“Stephen Nichols Interview” n. pag.).

\section{World of Warcraft and Advancements in Trade Affordances}

Blizzard's 2004 MMO World of Warcraft changes the methods of exchange between players by adding various technical interfaces, including auction houses, trade windows, and mailing systems. There is no shortage of information detailing various aspects of World of Warcraft, including its demographics, interfaces, ethics, mechanics, geographies, metagames, history, and social life. In fact, I find it difficult to write about World of Warcraft due to the sheer amount of information available on it. It is incredibly influential, and dictates many conversations about MMOs and their design. Miguel Sicart explains that "World of Warcraft is a symbol of dominant trends in virtual world 
game design. Its tremendous success, grounded in an excellent design and compelling environment, will most likely ensure that online worlds will look like it for some years to come" (187). As a result I will focus my description on one interface operation in the game, and centre my subsequent discussions around it: the auction house. The auction house is itself a significantly complicated interface that requires a rather detailed explanation. The auction house interacts with and around much of the research on World of Warcraft, and is a central component of game play. I will describe the auction house in detail below using information from the official World of Warcraft site, as well as World of Warcraft fan sites, guides, and wikis. ${ }^{5}$ I mostly refrain from interpretive commentary in this section because the action house's relevance becomes more apparent in the subsequent discussion.

The auction house is a place to buy and sell in-game items, from user-created products to found items of value. Auction houses are located throughout World of Warcraft's major cities, and can be accessed by numerous concurrent users. Items up for bid on the auction house are purchased with virtual gold, the game's official in-game currency, and are listed with a current bidding price, an amount of time before the auction expires, and a flat-rate buyout price. Gold placed on a bid is held in escrow until the auction is complete. If a user's bid is successful, items are delivered to their character through an in-game mailing system. Players can sort items in the auction house for specific items by name, sub-categories such as armour or weapons, required level for use of an item, items by profession (such as blacksmithing, enchanting, mining), item rarity,

5 Official and unofficial World of Warcraft sites use auction house, the auction house, and auction houses interchangeably and synechdocally, as does this dissertation. Generally speaking, the auction house denotes its broad scope as an institution within the game, and the plural auction houses refers to various locations of an auction house within the game. 
and time left on auction.

WoWWiki, an enormous fan wiki which claims four to five million users per month, details numerous lore and game play details for users, including using auction houses. The wiki describes three distinct methods for earning gold through the auction house: farming, crafting, and trading. Like many other games, players in World of Warcraft can have numerous professions and skills which they develop, and one set of these in World of Warcraft pertains to collecting resources that are used by other professions. Farming is the practice of collecting resources, through professions such as mining, and selling these raw resources on the auction house. Crafting uses the resources acquired from farming to enchant, inscribe, or create items, which can also be sold at the auction house. Players can choose only a few skills, and items which work as ingredients in one skill might require a second skill, and thus a second player, to acquire and trade this item. As a result, players are encouraged to trade items and contribute to the broader economy by virtue of the game's rules regarding skills. One player in need of metals for blacksmithing might not have a mining skill, for example. Finally, trading, as WoWWiki describes, is the "most advanced way of making gold. It includes any form of buying and then reselling goods. [Trading] has the greatest potential for earning a lot of gold/hour, but requires very deep understanding of [the] market in general and [a server's] market specifically" ("Working the Auction House" n. pag.). The term goldfarming actually refers to all three of these practices and more, as Nick Yee (2006) and Julian Dibbell (2007) explain, but their reports on the issue describe only the first practice in detail.

One of the major reasons the farming method was discussed more frequently than 
the other auction house methods during Yee and Dibbell's period of study was the inability to sell certain materials or services on the auction house. For example, enchanting, one of the forms of crafting, had to be done through player-to-player interaction. As an example, players formerly employed their own chat macros to optimize their exchange of goods and services outside the auction house. Macros are used for combining multiple actions into a click or key bind, thereby executing useful scripts to facilitate efficient use of time. These can be used for game play purposes or chat purposes. For the purposes of trading, macros were often built to communicate in chat. For example, a historic chat macro prior to the 2.0.3 patch update in 2007 might be written as follows:

"'

12 selling enchants in front of the bank in org(rimmar):

$/ 2$ icy (price)

$12+20$ int to staff (price)

$/ 2$ byom $=15 \%$ mark-up

Similar to other MMOs, World of Warcraft contains chat channels, and $/ 2$ is the command to communicate in the official trade channel. This macro is designed to spam the location of the enchanter (the bank in Orgrimmar), what they are selling (an enchantment on an item which increases the intelligence attribute of a character), and information on the mark-up cost for players who bring their own materials (byom). There are various ways to construct chat macros, as well as java code to output these 
macros, and numerous forum posts, sites, and guides for building them. Players could use these macros to efficiently advertise or communicate services. However, the majority of materials (mats) would still be purchased and sold at the auction house from users farming them. Players would then meet in the same physical location, and use a trade window to manage the exchange of enchantment for gold or items. In the 2.0.3 patch mentioned above, however, Vellum's were introduced, which are materials that allow an enchanter to bestow an enchantment on them for later transfer to another item. This patch allowed enchantments to be sold on the auction house, and subsequently eliminated the need for chat macros that spammed the price and location of an enchanter. Various patches, such as 4.0.1 in 2010, for example, have similarly increased the accessibility and use of the auction house among professions, rendering player-to-player trade unnecessary for most professions.

The updates to the auction house have, as a result, altered the methods used by gold-farmers, but the name stems from the method of farming resources for sale on the auction house. The auction house has grown to encompass the majority of trading practices since the game's inception. The auction house is central to the game's economy, and WoWHead, a strategy and news site for the game, describes it as follows: "it is the Azeroth stock market [players] all invest in. Most importantly, it is the foundation for [the] economy. Now, mastering the foundation of an economy is next to impossible in real life, as there are thousands of aspects of it. But in Warcraft, players have only to master the auction house" ("Making a WoW Fortune" n. pag.). As a result of the auction house's relevance to the game's economy, this dissertation examines this trade interface in relation to broader concerns regarding capitalism and video games. 


\section{Reamde and the Growth of Digital Trade}

Neal Stephenson's Reamde is similarly concerned with the relationship between capitalism, video games, and labour. The novel's plot centres within and around the fictional MMO T'Rain, and articulates the integration of game play, particularly as it relates to trade and economy, into everyday life. T'Rain's most notable characteristic is the sheer totality of its world: the game's language, mythology, culture, geography, and economy are designed to rival their authentic counterparts. For example, the novel's protagonist recruits linguists to create a complete language for the game, and geologists to construct accurate representations of the landscape. Players within the game are even described as embroiled within various class and culture wars. The goal of the T'Rain's mimetic design, however, is economic trade. Unlike real-world MMOs, T'Rain gears its players towards profit-generating activities and encourages real-money transactions, the exchange of in-game currency or items for real-world currency. The novel's protagonist becomes wealthy by capturing the labour of players and dissolving the separation between digital and non-digital currencies. Reamde's fictional MMO encourages and enables gold-farming explicitly.

Stephenson's novel furthermore accounts for the shift from something like World of Warcraft's auction house to the Steam Market. The novel is derivative of MMOs from the 2000s with regards to its descriptions of game play and design, but anticipates the development of game markets intentionally developed to accumulate real-world wealth. Reamde describes the emergence of game economies in the global market, demonstrates 
the influence of these economies on national currencies, and fictionalizes how game economies might affect personal and political relationships.

Writing about Stephenson's novels in general, Christopher Hellstrom (2015) documents particular tropes characteristic to Stephenson's work: "1) A celebration of geek culture and techno cultural observations 2) The privileging of makers and doers over pure abstract thinkers 3) Physical action and 4) One or two "big ideas" that fuel Stephenson's interests and creative energies" (Alpha Geek 68). Additionally, critics have noted that Stephenson's work is broadly concerned with "the third culture," a term coined by Jonathan Brockman (1995) to describe the process of communicating scientific information in an accessible fashion. Andy Clark's Natural-Born Cyborgs (2003) refers to Stephenson's work as participating in the third culture, as well. The third culture traditionally constructs science as an edifying institution for correcting philosophical debates. Hellstrom, however, rejects the interpretation that Stephenson's work positions scientific information as the primarily discipline, and insists instead that Stephenson's novels champion a unification of sciences and humanities. In Stephenson's Snowcrash, for example, the protagonist, Hiro, is able to navigate successfully between the disembodied Metaverse and the real world.

Despite elements of hard science fiction, Stephenson's work is also concerned with culture. While a nauseating amount of time is dedicated to accurately describing and detailing various technologies in the novel, Reamde spends a fair amount of time demanding readers seriously consider the ethical, political, and global implications and influence of expansive video game economies. However, Stephenson's novel ultimately tends to favour the same type of individual that his novels favoured in the early 1990s. 
Richard Forsythe, the novel's protagonist, is able to navigate and control various worlds successfully, including his family gatherings in Iowa, his social media presence, his illicitly generated income (both in- and out-of-game), and the game world he plays in and operates. The ability to manage and synthesize information, cultures and technologies is one of Forsythe's primary qualities. Similar to the Steam Market, T'Rain's economy, culture, and social life are closely managed and regulated in order to produce wealth.

\section{The Steam Marketplace's Organizational Structure, Esports, and Skin Gambling}

The Steam Community Market is a real money market for purchasing and selling items associated with Steam games. The Steam Market was added in 2011, according to an official posting on their website, to facilitate trade and allow players to acquire items from games featuring in-game economies. These items include various cosmetic items, which have no direct influence on game play, such as skins, as well as trading cards and other collectible items related to games. Items are sold for a set price listed by the seller, and items are purchased using money from the Steam Wallet. The Steam Wallet is a personal account which lists its users amount of money according to their national currency, generally, and can be added to through PayPal, gift cards, or credit card. There is no virtual currency system or exchange rate, unlike, for example, real-money purchases of gold in World of Warcraft. Adding, say, \$5 CAD will result in \$5 CAD available in the Steam Wallet. The current maximum amount one can add to a Steam Wallet is \$2000 USD. The Steam Wallet and other restrictions make item purchases generally safer than traditional real-money trading done through secondary markets, but also ensures that the 
money stays in Steam's economy as there is no way to convert money from a Steam Wallet back into other currency. The Steam Market can be imagined as an updated auction house from World of Warcraft, in which real-money transactions replace in-game currency as the method of payment.

The Steam Market is included in the Steam software, and buying and selling works as follows. A player can enter the market by clicking on the associated tab. From there, a player can choose to purchase or sell items. In order to purchase an item, players can search for in-game items by game, price, description, or specific name. That player then pays the associated fee and the money is taken from their Steam Wallet. To sell an item, players can search their own inventory, an aggregate of all in-game items available for trade across all Steam games, and list a price. Clicking an item for sale will also display a graph and other information regarding that particular item's sale history, peaks and valleys in prices, and average current price, as well as the lowest current selling price. Users list items for sale by entering the Steam Market where there are two input boxes for determining prices: "buyer pays" and "you receive.’'If a user simply fills in "buyer pays" it will automatically fill in the "you receive" portion, but not the other way around. The reason these are separate is because of the Steam Transaction Fee, which, according to the official Steam FAQ, is a surcharge "collected by Steam and is used to protect against nominal fraud incidents and cover the cost of development of this and future Steam economy features. The fee is currently $5 \%$ (with a minimum fee of $\$ 0.01$ )." The buyer pays the transaction fee. Moreover, three games currently have a transaction fee of 10\%: Dota 2, Counter-Strike: Global Offensive (CS: GO), and Team Fortress 2. Notably, these are three of the most popular games on Steam, and also three games with 
enormous in-game economies.

The Steam Market is a method for trading game items through a medium of exchange (national currency in this case). Steam has bypassed the necessity for item trades through any form of game currency through the use of real money transactions. Nevertheless, players have also developed a form of currency to facilitate trade on their own as well, without using the Steam Market. Steam Keys are flat-rate items available for purchase through Steam at unlimited quantities for \$2.49 USD. These keys were originally developed for Team Fortress 2 as "Mann Co. Supply Crate Keys," but have become colloquially and commonly referred to as Steam Keys for their use as currency. $C S$ : $G O$ skins, for example, are acquired by opening a crate with a Key. Keys can be used to unlock crates and boxes which contain cosmetic items for various games. Additionally, weapon cases (crates) and low-value skins in $C S: G O$ are randomly dropped to a random number of players at the end of a game. These drops generally sell for a few cents, but ensure all players have the chance to participate in $C S$ : $G O$ 's economy. More importantly, however, they encourage players to purchase a Key for $\$ 2.49$ from Steam in order to open these weapon crates.

In any case, players realized they could use these Keys as a set form of currency throughout the market, and began trading items for Keys. Mitch Bowman documents that in 2012, for example, cosmetic Earbuds in Team Fortress 2 sold for around 25 Keys (“The Hidden World of Steam Trading" par. 12). Steam Keys are used not only for trading and gambling, but also as a method of bypassing trade restrictions on the Market of selling items valued over $\$ 1800$ USD. Despite these advancements, there are several third-party sites for real-money trading of Steam game items, Keys, crates, and even 
games because of the conversion restrictions of the Steam Wallet. Bowman notes that players "sell items for real-world currency that they send via PayPal or Bitcoin instead of trading for other in-game loot via Steam's official channels. A network of third-party sites like TF2 Outpost and backpack.tf has formed to facilitate these unofficial trading arrangements" (par. 64). Moreover, Bowman notes that these third-party trades have been sites for numerous scams and credit card fraud activities. Steam has, generally speaking, offered no support for victims of third-party scams because the exchanges that take place within the actual Steam application programming interface (API) require multiple confirmations on both sides.

Security is one of Steam's major concerns regarding trading and the Market, and as such there are numerous restrictions in place. Perhaps above all else, in fact, the Steam marketplace is ruled by security. Players are required to provide personal information for easy access to marketplace sales, or must wait until clearance is given through emails and waiting periods. Steam's security is indicative of how important efficient exchanges between players is to their production of value. In fact, regulations other than security are quite minor within the marketplace. Here is a list of various Market restrictions listed on their support site:

- Items purchased from the Market cannot be traded or sold again on the Market within 1 week of purchase.

- $\quad$ Account must have a successful purchase older than thirty days, but not older than a year.

- $\quad$ Account must have had Steam Guard enabled for 15 days.

- If you have had your password reset by Steam Support you will need to 
wait another 15 days with Steam Guard enabled before you can trade or list items on the Community Market.

- If a chargeback has occurred on your account you will be eligible 30 days If a password is reset on an active account, trading will be restricted for 5 days. If an account has not been active within the past two months, this restriction will last for 30 days. This does not affect password changes, only password resets.Adding a new purchase method to an account will trigger a trade and market restriction for 7 days. Accounts under trade or VAC bans cannot trade or use the market. ${ }^{6}$ ("Trading and Market Restrictions")

Furthermore, all purchases are final and players have no right to demand refund or refusal of payment upon completion of the transaction. Additionally, whether a game has ingame items for purchase on the Market is up to the developer, who receives some percentage from the transaction fees along with Steam.

The Steam Market also allows users to trade items or Keys directly with one another, and this practice forms the basis of contemporary skin gambling. The trade affordances of the Steam Market have resulted in an enormous gambling market in which virtual goods on Steam operate as a form of virtual currency. As the Wikipedia entry on "skin gambling" explains, the Steam Market "can be interfaced by third-parties to enable trading, buying, and selling of skins from players' Steam inventories for real-world or digital currency." When I first proposed this dissertation, I was nervous about my ability to access information regarding the close connection between Steam trading and gambling. However, twitch.tv users have since uncovered various unethical business

6 Steam Guard is a secondary security function that requires users to verify their accounts through email or text. VAC refers to Valve Anti Cheat program responsible for detecting various in-game cheating programs or hacks. VAC bans can be anywhere from a week long to permanent. 
practices taking place on several prominent $C S$ : $G O$ gambling websites. As a result, various news outlets and journalists have reported on this aspect of the Steam Market ("Skin Gambling"). In July of 2016, it came to the public's attention that two YouTube users and one Twitch streamer who promoted and advertised for CS: GO Lotto, a skin gambling website, were also the site's owners and operators. None of these users disclosed this information. Skin gambling happens, generally speaking, in one of two ways. The first is users betting on the outcome of professional esports matches, which I will address in more detail later in Chapter 3, and the second is through digital randomnumber generators. Essentially, players could place skins as bets on digital lotteries or slot machines and gamble accordingly. It is possible to view upcoming winning numbers in gambling programs, and the CS: GO Lotto operators were streaming themselves winning enormous bets as if by random chance while actually knowing the upcoming results.

While the Wikipedia entry notes that "when the existence of the skin gambling situation was discovered in mid-2016, estimates for the economics of skin gambling market had dropped, but by early 2017, these analysts found the market did not drop as much as they expected, and with gambling sites still open and growing." It should be clarified here that this entry's reference to the existence of skin gambling being discovered refers to the various lawsuits filed against Valve in addition to various gambling sites, as well as the rise in government restriction on the use of virtual goods for gambling. For its own part, Steam has been keenly aware of the existence of gambling for a number of years. It is common for users to make bets on the outcome of their own games against one another as well as professional esports matches. In 2015, 
iBuyPower, a professional $C S$ : $G O$ team, were permanently banned by Steam for match fixing. The players intentionally lost a match in which each iBuyPower player bet approximately $\$ 20,000$ USD in skins against their own team winning. They were playing against what many viewed as an inferior team, and their easy defeat made various people suspicious. As a result of an investigation by esports journalist Richard Lewis, Steam began tracking the exchange of items between various accounts, and determined that iBuyPower had worked in conjunction with gambling site CS: GO Lounge to throw the match. Despite this, it was not until various government officials and legal lawsuits were filed that Steam began to take action.

On 13 July 2016, Erik Johnson made an official release on Steam regarding gambling and the Steam Market:

In 2011, we added a feature to Steam that enabled users to trade in-game items as a way to make it easier for people to get the items they wanted in games featuring in-game economies.

Since then a number of gambling sites started leveraging the Steam trading system, and there's been some false assumptions about our involvement with these sites. We'd like to clarify that we have no business relationships with any of these sites. We have never received any revenue from them. And Steam does not have a system for turning in-game items into real world currency.

These sites have basically pieced together their operations in a two-part fashion. First, they are using the OpenID API as a way for users to prove ownership of their Steam accounts and items. Any other information they obtain about a user's 
Steam account is either manually disclosed by the user or obtained from the user's Steam Community profile (when the user has chosen to make their profile public). Second, they create automated Steam accounts that make the same web calls as individual Steam users.

Using the OpenID API and making the same web calls as Steam users to run a gambling business is not allowed by our API nor our user agreements. We are going to start sending notices to these sites requesting they cease operations through Steam, and further pursue the matter as necessary. Users should probably consider this information as they manage their in-game item inventory and trade activity. ("In-game Item Trading Update")

A day later, the world's largest game streaming service, Twitch.tv, warned its users that promoting gambling sites was against their terms of service, despite the fact that many full-time streamers and professional players who streamed on Twitch were openly sponsored by various gambling sites (Twitch.tv/DaZeD). The API of the Steam Market is the primary enabler, by the developer's own admission, of contemporary skin gambling. The interface affordances of the Steam Market are designed for efficiency and security, but much of the value is generated through secondary features of play. I will demonstrate in Chapter 3 that Steam's trade interface is intentionally designed for the exact kind of entrepreneurial behaviour that Erik Johnson's post publically condemns. Steam issued a public statement regarding these gambling sites only in response to public condemnation and government investigation. Their banning of the iBuyPower team the previous year reveals their complicit knowledge and acceptance of these sites. Furthermore, that Steam 
was able to trace the exchanges of items through various accounts and associate them correctly with individuals demonstrates their own deep understanding of and control over their trade system.

In an interview with Doug Henwood, former Greek finance minister and Grexit figure Yanis Varoufakis recounts his time working with Valve. He explains that valve CEOs wanted to "blend together different gaming interfaces," and by doing so realized the necessity of combining currencies through different games ("Interview" 33:40-42:00). For a short time, Varoufakis ran a blog about his experiences at Valve entitled VALVEnomics. In 2012, during his time at Valve, Varoufakis explains there were approximately 90 million people and a billion US dollars interacting through Steam, although this number has certainly increased since his time, given the current worth and player population. Eben Novy-Williams and Joshua Brustein (2016) note that the amount of money spent on $C S$ : $G O$ gambling alone is currently generating billions of USD per year ("Virtual Weapons" n. pag.). In his blog, Varoufakis recounts the supposed anarcho-syndicalist nature of Valve's workspace, and the associated libertarian economic principles that guide the Steam Market. He refers to the economy at the time as a "barter economy" in which "astute traders" can accumulate wealth and explains that users can also design an app or cosmetic item and sell it ("To Truck, Barter, and Exchange?" n. pag.). Steam is a place for economic entrepreneurs as well as players, it seems.

Additionally, Varoufakis explains the level of management and mastery that developers had over their market: "Valve is like God, omniscient. [They] have access to all information at all times" ("Interview"). Varoufakis recalls that interference, rule changes, and experimentation are central to Valve's management of the Steam 
Marketplace:

Let's say there is an item that goes out of control, like a bubble, and when and if it bursts, people will be burnt. If you buy something and it loses value then you will be upset, and one of the things the game designers don't want is to upset them. So you can regulate it. You can do controlled experiments. You can apply different regulation regimes across games in order to study things. You cannot have markets without the state or states without markets. These companies find themselves with economies in their midst. ("Interview")

Invoking the metaphor of the state here positions Steam as a sort of global institution in relation to these states. Steam distributes games and helps manage their in-game economies through its market, but each game has its own developer and associated economy. In order to focus on a particular example, Chapter 3 examines the Steam Market's management of Counter-Strike: Global Offensive's online economy and surrounding competitive esports culture.

\section{Trading Games}

There have been various games that attempt to simulate trade and trade markets, and, for the most part, this dissertation ignores these games. This section will describe some of these games, and explain the exclusion of these games from this discussion. Instead of addressing games explicitly designed to simulate trade, this section will describe two games, Papers, Please and Recettear, which reflect critically upon the associations between play, labour, trade, capitalism, and governance. 


\section{Drugwars (1984), The Patrician (1992), Merchant Prince (1993), Master of}

Orion (1993), and Age of Pirates (2006), are several in a long list of trading simulation games that primarily deal with arbitrage in the simplest way imaginable. Players are given prices of items and markets to interact with, and encouraged to buy low and sell high, thereby producing profit. In the process, players acquire more wealth and resources with which they can leverage their purchases and exchanges. Writing about the banality

of these kinds of games, Sin Vega explains that the "old RPG treadmill" is replaced "with money and ships instead of XP and Swords" ("Art of the Deal"). Vega claims that most games about trade fail to account for the both the human and economic complexities that produce markets.

In Drugwars, for example, players begin with a set amount of money and inventory space. Players travel throughout a virtual New York City and check prices of various narcotics in each of the city's boroughs. Travelling from each area of the city uses one day of game time, and players are given thirty days to accumulate as much wealth as possible. Heroin, for example, might sell for a lower price in Manhattan than in The Bronx, but cocaine sells for a higher price in Manhattan than in the Bronx. Players then purchase drugs from cheaper locations and sell them in more expensive locations. This simple arbitrage system is complicated by technological and graphic advancements in later games like The Patrician or Merchant Prince, but the overall concept remains the same. In the Merchant Prince series, for example, players can bribe officials or hire thieves, but trade is done by shipping commodities from one major city to the next in order to profit from price imbalances among these commodities. The majority of games ostensibly about trade are actually stylized arbitrage simulators that fail to 
account for the institutional, technical, and interpersonal affordances that help shape markets and trade practices. As a result, these games are not discussed in significant detail throughout this dissertation.

These games represent expansive and extended markets in which buyers and sellers are numerous and currencies work to facilitate mass trade and engage in arbitrage. As a result, a simple truck-and-barter system is nowhere to be found in the majority of these games. Notably, trade affordances in the Steam Market, The Realm, and World of Warcraft result in markets that exist in a liminal space between economies of barter and the extensive, global markets these "trading" games represent. Players often create their own currencies, as previously noted, but also meet in-game to perform enchantments, trade, or communicate through various interpersonal communication systems to exchange skins or cosmetic items.

The two single-player games discussed in chapter 4, Recettear and Papers, Please, are less concerned with trade as a form of wealth accumulation than they are with the state of game play as a site of productive consumption and exploitation. Recettear satirically employs the same arbitrage system that trading simulation games employ while presenting a critique of capitalism. Recettear begins as an RPG where players take on the role of Recette, a young girl who has inherited her father's debt. A fairy named Tear, working on behalf of the loan company, teaches Recette how to run an item shop. The result is a typical arbitrage game except that, through its narrative, it critiques the exploitative capitalist practices these trade simulators promote.

Although Papers, Please is not overtly about trade, it asks players to make various ethical and ludic exchanges through its game play. The game presents moments 
of exchange as socialized and politicized acts with far-reaching consequences, while simultaneously commenting on the tedious and calculated decisions players must make in order to succeed in the game. Players take on the role of a border guard in a dystopian nation. The border guard's family is destitute and sick, and players are paid for each traveller that is processed through the border. Increasingly complex bureaucratic rules are added with each passing day, thereby limiting the amount of people who can be let through while effectively following the rules. As these rules dictate who may or may not pass through the border, any infraction by the player automatically results in lost wages. Additionally, players are offered bribes and other incentives to allow travellers through. Players are asked to make ethical decisions about these exchanges, as the mechanics and narrative come into conflict with one another. Papers, Please forces players to perform menial and repetitive bureaucratic tasks through its rule system while its narrative creates escalating tension and anxiety. By so doing, the game demands that players navigate between discordant mechanic and narrative consistencies. Both of these indie games stress the tension between efficient game play, which props up an exploitative system, and the ethical decisions players encounter. These games demonstrate the porous separation between social life, economics, and game play that various critics have defined as central to contemporary video games. Moreover, these games criticize the association of labour and play, while providing an aesthetic depiction of tensions that surround capitalism and video games.

The close relationship between gaming and capitalism is central to the discussions and close-readings throughout this dissertation. However, it is necessary to address the technical and theoretical research that inspire these criticisms before making them. For 
example, it is essential to provide research and interpretations of video game users, interface operations, and Marxist methodology. Therefore, Chapter 1 contains the literature review necessary for the close-readings made in Chapters 2, 3, and 4.

\section{Chapter Breakdown}

Chapter 1 discusses a variety of research both broadly and specifically related to trade interfaces. The first section provides a brief description of some demographics related to trade and MMO users. Additionally, it addresses some of the historical moments in MMO development and positions them according to their relevance to trade interfaces. Furthermore, Chapter 1 addresses economic theories which might help provide a terminology or method for thinking through the influences, justifications, and necessities for trade affordances. This chapter addresses the social, cultural, and political influences of interfaces, rule systems, and game mechanics. Chapter 1 explores the role of the player and presents a possible methodology for determining an ethical player-game relationship. The discussion of interfaces provides insight into player ethics and decision-making in response to these interfaces, as well as other rules and game mechanics. In this literature review, I also put forth the argument that players are expressive agents acting within the constraints of a game's rule system. Finally, the literature review addresses the Marxist philosophies that shape this paper's methodology and reading of trade practices and their developments. More specifically, my literature review outlines the Autonomist Marxist methodology that shapes the textual interpretation in the following Chapters. The literature review is designed to move 
toward a Marxist critique by synthesizing it with Miguel Sicart's view of ethical players, while rejecting neoclassical economic thinking about trade interface design and trade practice.

Chapters 2, 3, and 4 are a collection of interpretive readings of several games and one novel in light of the literature review, and largely inspired by a combination of cultural and material Marxisms, and Autonomist Marxism in particular. This dissertation describes the automation of trade practices as an attempt by capital to divide social relationships and manage production. As a result, trade practices become efficient and productive with regards to the creation of wealth and management of labour. In Chapter 2, I address the specifics of trade between players at the individual level. Specifically, I contend that exchange is a diegetic act in The Realm's MP system, and thereby constitutes a productive act of play. In this chapter, I also address the technological changes effecting interpersonal trade as a result WoWs auction house. It will become apparent that the rules of trade in The Realm were messy, inefficient, and inherently tied to various cultural and social practices. Chapter 3, which discusses Stephenson's Reamde and The Steam Market, marks a shift from interpersonal trade operations to large-scale or global acts of exchange within video games. Unlike The Realm, contemporary trade practices in the Steam Market are incredibly efficient and well-managed. My case studies suggest that Suits's claim that game rules prohibit efficiency for the sake of play was relevant to an era that is largely behind us. Finally, Chapter 4 analyzes games that critique the association between capital and play with regards to acts of trade. The indie games and novel under discussion also demonstrate the manner in which trade has become a site of labour divorced from acts of play. 


\section{Chapter 1:}

\section{A Review Concerning Economics, Interfaces, Players, and Ethics}

\subsection{Demographics to Set the Stage of Exchange}

MMOs contain players from across almost all demographics that have access to internet and computers capable of meeting system requirements. Players can be members of different economic and social classes, genders, and age groups. Statistics indicate that the majority of players tend to be male, although the degree of this majority varies from country to country (Nick Yee 2005, Bonnie Nardi 2010, Vili Lehdonvirta 2005). The median age of players tends to fall between mid-twenties to early thirties, although Taylor notes that older parents also play with their much younger children. Motivations for playing tend to be equally varied, particularly in MMOs. Competition, socializing and networking, escapism, entertainment and enjoyment are some of the listed reasons (Yee 2005, Nardi 2010, Lehdonvirta 2005). Some players are interested in simulated arbitrage, investing, and gambling on the game's market, while others associate purchases with social functions such as role-playing.

Many researchers have listed the social qualities of MMOs as one of the draws for many players. Bonnie Nardi (2010) explains that many users play with friends and family members and view the game as "an extension of their existing social lives" ( $M y$ Life as a Night Elf Priest 21). Moreover, while people extend their social lives into the game, the "virtual world itself is a stimulus to real world interaction" for making connections outside the game (21). In her ethnographic work on World of Warcraft, 
Nardi mentions that "study participants commented frequently in the interviews about the importance of socializing" (22). Researchers also identify the importance of MMOs as virtual communities defined by geographic space as opposed to chat rooms defined by text communication (Turkle 1995). Similarly, Nardi explains that “ [v]irtual worlds perhaps feel more authentically like cultures than chat rooms because of the elaboration of space and objects" (18). Virtual words are spatial, as are the large majority of games, but in a contiguous fashion: there is, as Pearce explains, "a sense of spatial continuity" (Communities of Play 32). MMOs, unlike other games, are persistent; they remain "on," so to speak, as the game world's economy, non-player characters (NPCs), and other players continue to develop, operate, and move while any given player is offline. Similarly, MMOs tend to contain "persistent identities" or avatars that represent players in the game world, and retain their characteristics and possessions between gaming sessions. Importantly, MMOs are also spatially and culturally inhabitable as well as populous, and therefore create unique cultural spaces, practices, and play etiquettes. Specialized locations, skill sets, items, and identities within MMOs help to produce distinct roles for players, thereby increasing the interpersonal relationships necessary for developing emergent or active player culture.

Guilds are one of the primary forms of communities that form within populated virtual spaces. Guilds are social groupings of players built around shared values or characteristics. There are guilds based on location, competition, religion, age, gender, real world friendships, family, and many other interests or associations. Guilds are often hierarchical in nature, with committed and entrenched players making decisions and leading others. There can also be organizational roles within guilds, such as raid leader, 
banker, or secretary, as well as dedicated skill and class positions, such as enchanter or tank. Some guilds are less concerned with hierarchy and are dedicated more toward roleplaying or social interaction, while others view the game as a cooperative, competitive process. For example, Lehdonvirta explains that World of Warcraft's "hard-core raid players are convinced that World of Warcraft is about succeeding in extremely difficult raids and doing it better than competing guilds" (Virtual Consumption 32). Although there exist shared interests or desires among guild members, they can also be diverse social groupings with loosely related interests that players join for support or advancement. Nardi finds that guilds can function as a method of unifying "social classes for authentic shared activity" (24). Guilds can at once be both inclusive and exclusionary social communities.

Because players often join virtual communities (the MMO itself) or guilds based on shared interests, these communities tend to reproduce and manage a set of values which helps determine play patterns (Pearce 2006, Sicart 2009). Miguel Sicart explains that although games are composed of unambiguous rule sets, players work to define their own experience, thereby creating social rules that govern play in addition to the game's formal elements (36). As an example, he describes something referred to as ninja-looting and the communal reactions to this behaviour:

When a player, individually and without permission, loots the monsters killed by a larger group of characters, her avatar's name is publicly exposed so that other players will not party with her. Players understand that even though ninja-looting is allowed by the constitutive and operational rules of the game, it is ethically problematic and so they have to create rules governing that behavior within the 
world. (36)

As a result, an individual who consistently engages in ninja-looting will become a social pariah and be unable to party with others, essentially barring them from completing or experiencing large sections of the game. There are numerous examples of communities attempting to manage deviant behaviour across MMOs. Similarly, trade practices are subject to social and formal rules, and communities manage trade experiences in surprising and interesting ways. For example, Stephen Nichols, a lead designer and creator of The Realm, explains that they allowed for thieves and scammers to be jailed by an NPC magistrate ("Interview" n. pag.). In the jail, players could not send messages or do anything fun. Despite their ostensive punishment, Nichols notes that certain members of the community saw trade scams as a means to play the villain: "[jailing players] encouraged the anti-social players to compete with each other for longer sentences. Deep down, I think we knew that would happen. But in any case, it didn't solve the problem" (n. pag). Players engage with both user- and developer-produced content to create what Pearce refers to as "emergent communities," which she defines as "disparate systems [that] have in common a display of collective behaviors and even collective intelligences that arise out of, and yet transcend the actions of, the individual parts or elements" (Communities of Play 42). Social behaviours, then, can produce unusual or unexpected interactions with formal rule structures.

As a result, trade practices have predictably resulted in mixed responses and opinions among designers and players. Players trade and consume digital items for similar reasons that they play: socialization, entertainment, or competition. Additionally, players trade for economic gain, evidenced by account sales and real-money trading. 
Player communities have managed trade differently in various games over time because players trade for disparate reasons. For example, the diegetic role-playing aspect of playing the villain in The Realm became a source of conflict among the community because it made trading a risky process as far as item security is concerned. Despite this, a guide written by an old player describes "trading in The Realm as a potentially dangerous, but potentially rewarding venture" (“Trading Guides!"). The community attempted to manage its own trade through MPs, thus creating a rule system similar to Sicart's description of ninja-looting.

According to Lehdonvirta, MMOs are the "first genre of online services where real-money trading began in a significant volume" (45). These exchanges, combined with growing in-game economies, resulted in tensions throughout communities regarding social status, ethics, and interpersonal relationships. Nearly all early MMOs were swords-and-sorcery style games where players create an avatar and complete repetitive tasks to advance their prowess and abilities. In so doing, they acquire items and materials indicative of their time spent playing the game, and thereby indicate a form of virtual cultural capital. Certain players felt what Lehdonvirta refers to as an "achievement hierarchy" in regards to their material possessions and high-level characters. High ranking players in this hierarchy could be thought of as experienced players who have contributed to the growing social structures and economies of the game. Some players felt that these qualities ought to command respect among the community and developers.

However, Lehdonvirta explains that "very soon, or in some cases from the very start of the game, this idyll was unsettled by so-called secondary markets" (45). Some high-level players who wanted to quit the game or simply wanted cash realized the value 
of their time spent acquiring items and building a character. As a result, they began to search for buyers on secondary markets. Instead of labouring through the repetitive grind, players with expendable money were able to purchase these items and accounts. Lehdonvirta recounts that many players expressed outrage over these exchanges. Many players felt that this was a form of cheating because people purchasing accounts and items had not earned them on the same playing field as everyone else. Lehdonvirta also explains how this contributes to a breakdown of the traditional social order: "when this happens in a large scale, the traditional link between achievement and avatar is broken, and it becomes impossible to 'read' a person's social status from their possessions. While commodification preserved the material function of virtual goods, it was stripping them of their meaning" (46). Developers responded to these complaints among players by creating rules against certain types of real-money and account trades. EverQuest, for example, deemed the exchange of accounts illegal under the EULA (the account would be deleted if caught), and began tracing suspicious exchanges and name changes under accounts.

One particularly interesting result of standardized trade systems is the anxiety produced amongst Western players over the "Chinese gold-farmer." Gold-farming is the practice by which players acquire, through repetitive tasks over long hours, in-game currency and then trade mass amounts of it for real-world currency (usually for USD or Euros). Nick Yee explains that many gold farmers are not Chinese, although a large percentage of gold farms do exist in China. Gold-farming is practiced in many other countries, however, including Romania, Mexico, and the Philippines ("Yi-Shan-Guan" n. pag.). Yee attributes the stereotypical distinction of Chinese gold-farming to racism 
stemming from both American anxieties during the nineteenth-century gold rush and new anxieties surrounding the loss of American jobs: "the theme of immigrant workers being harassed by Westerners who feel they own the land and can arbitrate what constitutes as acceptable labour is one that is hard to escape." He explains that gold-farmers typically work sixteen hour shifts and have a daily quota of in-game currency to acquire. Furthermore, these workers are employed by bosses who own the machines and games the workers play on, and show up to work at a space filled with other workers. Yee notes that many of these workers have referred to their work places as sweatshops.

Furthermore, owners of gold-farming sweatshops are usually contracted out by Western companies who then sell the in-game currency back to Western players. Epictoon.com, a western gold-selling site, explains their mission statement as follows:

Many gamers enjoy the benefits of Real Money Trading (RMT) and are frustrated with the bad service, terrible English and spam-and-scam business strategies of the Chinese gold sellers. EpicToon provides you with a safe and dependable alternative. Our mission is to become your trusted source for virtual currency through honest and reliable business practices, great pricing and good old fashioned service. Like you, we believe in the benefits of free market competition. ("About Us" n. pag.)

In April of 2014, EpicToon merged with IGE.com, another online currency dealer ("IGE and EpicToon Announce Formal Service Agreement" n. pag.). What EpicToon and IGE conveniently forget to mention is that they purchase their in-game currency from Chinese gold-farming businesses. In 2006, IGE's purchase rates were publically available, and are still available under archive on Yee's Daedalus Project: 
To give you a sense of where most of the profits go, on WoW's Suramar server, IGE will buy 1000 gold for 25 USD. They sell that same 1000 gold for 66 USD. So for every dollar spent on buying virtual gold, only 37 cents of it goes to the actual gold farmers. The gold farming industry is not only driven by Western demand, but most of its profits in fact also go to Westerners. ("Yi-Shan-Guan" n. pag.)

These types of mass currency exchanges are not possible without trade interfaces. Without in-game trade affordances, players would have to drop the gold at a specific location within the game world or have a third party deliver it after picking it up in avatar. Trade interfaces allow for mass transactions, making it much easier to supply aggregated currency. It is Western companies that own the vectors through which informational currency travels, and these same companies engender racism among their clients in order to ensure their continued exploitation of underpaid and abused workers.

The desire among Western players to purchase in-game currency derives from a particularly individualistic sense of gaming identity. Thomas Malaby's Making Virtual Worlds (2009) is an ethnographic work on Second Life that describes capitalist pathology in contemporary gaming. Second Life is a social MMO, without explicit thematic elements, yet its mechanics explicitly encourage a particularly individualistic approach to game play similar to that of other MMOs. The game is largely about its content, which Malaby explains is a term in the gaming industry "used to connote, loosely speaking, the 'stuff' that is in the games that their users encounter or interact with. It is at the centre of discourse and practice about virtual worlds for both their makers and their users because the 'quality' of a world's content is often seen as an index of its appeal" ("Culture versus 
Architecture" 233). As in other MMOs, acquiring gear (of increasing tiers of quality and rarity) remains important in Second Life, but unlike other games, items are user-designed and -generated, from lawn chairs to buildings to art. Malaby refers to Second Life's social world as "architecturally restricted to an expressive individualism, where what one creates is conceived not only as content in a specific sense but furthermore as the realization of an individual user's creative desires" (236). Mastering these game mechanics or content tools, Malaby indicates, is "an important concept for understanding the ideological underpinnings of Second Life's architecture because it points in another way to the status of the individual and furthermore helps us to identify what kind of learning Second Life prompts . . . and what kind it ignores" (238). Malaby writes about purchasing a small piece of land in the game. The piece is sizable enough that he began to wonder what he ought to do with it. He began thinking about providing a place to sit down, and decorating his area with a tower-like structure and a bridge. He explains that not having this sort of space would represent a failure for him as a player, and that acquiring this space and its decorative items would "be more likely to present [himself] as a knowledgeable, competent user of Second Life - something other than a "newbie"' (241).

A common trend among contemporary video games is the decline of old subscription-based models, found in games such as World of Warcraft, The Realm, and EverQuest. Increasingly, these old models are replaced by free-to-play games that derive income from prestige items and in-game or in-app purchases, evidenced by cosmetic skins and pay-to-win game models. Neal Tringham (2015) explains this shift:

While selling virtual items to players of games that are initially free was always 
the dominant business model for social games, it has also become much more common among MMORPG developers. This approach was first popular in South Korea and China, and subsequently spread into the West through such games as Jagex's RuneScape; in the early 2010s, it has become the most common way of funding massively multiplayer games. Typically, the most popular works will still charge a monthly subscription, but games that have become less fashionable, or that cater to a more casual group of players, will be free, with their expenses paid for by the sale of imaginary goods (such as a better engine for a player's virtual starship, or a more attractive uniform for their game-world persona). (Science Fiction Video Games 223-224)

In 2015, even World of Warcraft, in a seemingly flagrant contradiction of their own EULA, which forbids players from exchanging in-game items or accounts for real-money transactions, created a token which could be purchased for real money and exchanged for in-game gold.

Moreover, the commodification that Malaby, Yee, Tringham, and Lehdonvirta describe indicates the emergence of a new market for virtual goods. Trading items and accounts for money is only possible in significant volume with trade interfaces that make exchanges efficient and reliable. Decisions to restrict real-money trading is only necessary because of trade affordances that permit players safe and easy exchanges. Interestingly, the developers sought to maintain a social hierarchy that was collapsing as a result of the behaviour their rule sets enabled. Of course, it is probably safe to assume that it was not obvious to designers or players why these trade practices emerged because they simultaneously constructed rule structures and interfaces at odds with one another. 
Additionally, the development of each trade affordance under discussion in this work is determined by technological affordances outside of the game itself. The Realm, as previously mentioned, was released and designed with dial-up internet in mind, whereas the always-on broadband internet and server storage capabilities of World of Warcraft allow for trade volumes impossible under previous technological advances. Additionally, the Steam Market is enabled by SMS and cell phone securities that govern its trade practices.

In the sections discussing economic theory and interface, this dissertation addresses research which explains some of the nuances behind these influential interfaces. Additionally, the forthcoming literature review will provide insight as to why players and developers might simultaneously support trade interfaces while failing to recognize their increasingly complex social properties. In particular, economic assumptions about the individual's role in the market provide justification for trade interface design, and institutional theory indicates a desire for increased economic consumption and trade.

\subsection{Economic Perspectives, Old and New.}

Transactions involving goods, services, information, or currency takes place throughout the world in various forms. Virtual worlds are no different, and are filled with people transacting products through agreements and coordinated transactions. In this section, I will address some economic and theoretical ideas regarding trade while gesturing toward specific examples from my objects of study and other games. I briefly 
highlight some of the basic economic assumptions surrounding trade and economic agents. In particular, I address philosophies from classical economic philosophers that have influenced contemporary ideas surrounding trade. Additionally, I consider New Institutional Economics as a method for theorizing and describing the emergence of trade interfaces. New Institutional Economics, while not without its shortcomings, provides insight into the economic systems that govern trade. Additionally, I gesture toward the desire among many economists, including neoclassical and New Institutional, to reduce the risk and costs of exchange. Trade-windows, auction houses, and in-game item delivery systems work to ensure the security and efficiency of exchanges, thereby aligning themselves with the overarching goals of various neoclassical and neoliberal economists.

\subsubsection{Classical Economics and Trade Motivations}

For classical economists, trade is a by-product of consumption and production. Adam Smith writes that "consumption is the sole end and purpose of all production" (Wealth of Nations 230). By this nature, trade is the method by which individuals enable consumption. John Stuart Mill contends that individual trade preferences arise from agents' abilities to derive use from the objects they acquire. Moreover, Mill claims that

utility is the primary incentive for people to trade: people make exchanges which result in satisfying needs or desires. Essentially, traders make decisions to acquire items that they see as satisfying whatever needs they prefer or prioritize. Trade, then, is a byproduct of enabling consumption between individuals or groups attempting to manage the results of 
their production. Trade is related to demand, desire, and the perceived utility of various goods by those engaged in transactions. Classical and neoclassical economists tend to view trade as a method for increasing consumption, a condition they believe is indicative of overall economic health. If consumer welfare is growing then the economy will function. In order to achieve these goals, classical economists concerned themselves with national and international policies related to the role, if any, the government has in protecting the nation-state's economic well-being.

Smith believed that free markets were a means to abolish monopolies, thereby freeing individuals from indentured servitude and debt. For Smith, government and employers colluded to gain an advantage in the market. Discussing "trade or mercantile" and protectionist systems, Smith explains that

In England, indeed, a charter from the king was likewise necessary [to trade]. But this prerogative of the crown seems to have been reserved rather for extorting money from the subject, than for the defence of the common liberty against such oppressive monopolies. Upon paying a fine to the king, the charter seems generally to have been readily granted; and when any particular class of artificers or traders thought proper to act as a corporation, without a charter, such adulterine guilds, as they were called, were not always disfranchised upon that account, but obliged to fine annually to the king, for permission to exercise their usurped privileges. (132)

Smith viewed economic protectionism as a form of state collusion by which corporations or agents were propped up in order to contribute to government wealth. Ultimately, classical economists desired a free market governed by what they saw as individual 
liberty and rationality. It makes one wonder how these early imaginations of individuals in the market would regard something like case openings in $C S$ : $G O$, which gesture towards an amalgamation of play, gambling, utility, desire, and stilted reasoning. At any rate, these thinkers pioneered free market ideologies adopted by later neoclassical and neoliberal economists.

However, it is essential to note that classical economic philosophies are weathered by history that precedes and follows them. Ernesto Screpanti and Stefano Zamagni explain that "European expansion and 'discoveries' of new land revolutionized how nation-states began to view wealth. Nations began to analyze their trade balance with one another, and subsequently adjusted their policies in order to accumulate more wealth" (An Outline of the History of Economic Thought 35). As a result, the state began to view itself and its subjects as a single political entity, resulting in what Smith and later economists like Murray Rothbard would call mercantilism. Thomas Smith the Elder, identified by Rothbard as the first mercantilist, believed that "self-interest is —a natural fact of human life to be channelled by constructive policy rather than thwarted by repressive legislation" (An Austrian Perspective on the History of Economic Thought 281). In an interview with Jacobin, however, Elizabeth Anderson explains that classical economists' assumptions about individual economic freedom were "plausible in the eighteenth century, [but] falsified by the technological innovations that brought about the Industrial Revolution" ("Where Despots Rule" n. pag.). Twentieth- and Twenty-First century proponents of free trade, such as libertarians from Rothbard's camp, continue to promote ideas from classical economists while failing to recognize that fundamental changes in production, division of labour, and class relations have thoroughly warped the 
potential for liberating wage-labourers. Anderson summarizes as follows: "What started out as a liberating ideology with some grounding in empirical reality has generated into a pipe dream marketed by hucksters with the aim of draining the meagre assets of their rubes" (n. pag.). Varoufakis's description of Valve as an anarcho-syndicalist company is partially inspired by this sort of ideology. The economic freedom granted, ostensibly, by the Steam Market is similar to what Anderson describes as the multi-level selfemployment scams propped up the Republican Party. Enterprising players, Varoufakis notes, can "play" the Steam Market like the stock market by purchasing items and hoping their value increases ("To Truck, Barter, and Exchange" n. pag.). But, Steam does not have to play its own market, because they are economically positioned to benefit off of everyone else's involvement, regardless of the outcome of any particular savvy trader's investment.

In many ways, these early economists set ideological standards for discussions about trade. In particular, they contribute to beliefs about individual property rights and ostensibly free economic flow between agents that shape discussions of economics today. Theoretically, interface operations like trade-windows enable the free exchange of private property between agents. Additionally, they also contribute to the efficient consumption of resources, thereby, according to classical economists, increasing the overall economic health of the market. Moreover, classical and neoclassical economists tend to view markets, and trading within said markets, as a calculable, impersonal act bereft of social interaction or culture. The idealized, equalized market contains rational traders looking to increase their utility (i.e. their profit or other method of satisfaction). The problem, of course, is that trade is always affected by interpersonal relationships, social structures, or 
institutions.

In neoclassical economics, trade can be similarly described as a byproduct of consumption and market activities. However, neoclassical economics complicates and expands upon ideas regarding about the free market, supply and demand, and individual actors. Vili Lehdonvirta notes that modern economists assess the worth of any given good by looking "at the amount of other goods the consumer is willing to forgo in order to consume it. This reflects the notion in modern economics that value is subjective" (Virtual Consumption 15). Moreover, neoclassical economics see human actors as fully rational individuals whose optimizing decisions, given a perfectly competitive market, result in equilibrium. Spithoven et al. explain that neoclassical economics strives for perfect competition, which "assumes equilibrium in a market, whereby all consumers maximize their utility and producers maximize profits. Consumers take their preferences (utility) as a given, and producers take the production technologies as a given" (Institutional Economics 45). ${ }^{7}$ In neoclassical economics, trade is an efficient and optimal exchange of items within a large market of buyers and sellers with defined and enforced property rights. Additionally, trade is determined by the supply and demand of items within the market. Varoufakis invested much of his research on the Steam Market into the failed dream of economic equilibrium under capitalism. Before calling the blog quits after a few short posts, he proclaimed, "the beauty of digital economies is that we have all the data at our disposal. We can actually observe how far or how close to

7 Spithoven et al. define market equilibrium for neoclassical economics as a state of equality between the "aggregate demand of individual consumers and aggregate supply of individual suppliers. The fully rational individuals receive through prices the information they need to make the right decisions" (44). In the perfect neoclassical market, "products are homogeneous and perfectly substitutable ... nobody has market power ... everybody has all relevant information about price and quality ... there are no barriers of entry or exit ... public goods and externalities do not exist" (45). 
equilibrium our economies are" ("Arbitrage and Equilibrium in the Team Fortress 2

Economy" n. pag.). Of course, none of these assumptions about markets seems all that applicable to virtual worlds and their markets, given the murky nature of private property in online worlds, transitive cultures, social norms, and uneven distribution of information and play skill among players.

Yet, Edward Castranova (2007) has written at length about his attempts to apply the basic principles of micro- and macroeconomics to virtual worlds. Castranova's early paper "On Virtual Economies" documented his realization that goods and services in MMOs operated under the law of scarcity, and much of his later work is built upon analyzing forms of scarcity in virtual worlds using neoclassical economic models. Castranova collected data on various economic activities surrounding EverQuest, and was able to compare the price of goods in secondary markets (eBay, for example) in order to determine an exchange rate of EverQuest's currency to that of the US dollar. By so doing, Castranova also extrapolated information about the game's GDP and "minimum wage" of its players (Exodus to the Virtual World). One troubling data point Castranova collected from the sale of avatars in various online markets was that, on average, female avatars sell for 40 to 55 dollars less than male avatars ("The Price of 'Man' and 'Woman'" 27). This particular data point demonstrates the ability, at the very least, for basic economic principles to provide a form of evidence about the broader cultural problems surrounding gaming culture to present. ${ }^{8}$

Nevertheless, Castranova eventually concluded that many economic assumptions

8 Here I am thinking about the violent and abusive actions of GamerGaters directed at figures like Zoe Quinn, Anita Sarkeesian, and others who demonstrated the virulent sexism so rampant within games culture and the game industry. Interestingly, their interpretations and readings of mechanics, narratives, and tropes are dismissed by their detractors as subjective opinion, yet Castranova, as far back as the early 2000s, clearly identifies economic evidence that something is amiss in the gaming world regarding gender equality. 
or theories were not applicable to virtual economies. In particular, he came to reject the idea that video game economies might be thought of within a supply and demand model ("Virtual Worlds" 17). Additionally, he found problems with the microeconomic models in neoclassical economics that players acted rationally regarding economic activity like trading. According to neoclassical microeconomic models, an increase in the amount of available goods is a desirable quality in the market. Yet, Castranova indicates players in EverQuest became disillusioned at their own increasing affluence and the increase in available goods, which resulted in a decrease in the price of items both in-game and in secondary markets, which in turn resulted in a decline in trading ("Virtual Worlds" 17). According to Lehdonvirta, the same economic abnormality happened in early MUDs, resulting in what he calls mudflation: a term that "could be defined roughly as a situation where the aggregate amount of goods in circulation increases faster than the number of consumers" (18). Lehdonvirta notes that modern economists would celebrate this, in the real world, as economic growth. Castranova and Lehdonvirta both identify, however, that users prefer meaningful game play challenges over economic growth, and players desire competition and reward more than mere economic satisfaction. Note that economic satisfaction and meaningful game play experience are not mutually exclusive, however, and are often closely intertwined. As I will demonstrate in the chapter about The Realm Online, economic activities can be thoroughly connected with satisfying game play experiences at the diegetic level.

\subsubsection{Trade Affordances as Institutional Forms}


Ultimately, both neoclassical and classical economics fail to fully describe the transaction behaviour found within MMOs, and as a result other economic models are necessary to describe the complexities of virtual trade. There are, however, some neoclassical foundations upon which an accurate description of trade processes might be built. New Institutional Economics (NIE) provides a model that might help to describe the development and function of trade interfaces such as auction houses within contemporary MMOs. NIE is a multidisciplinary approach to economics that, ultimately, attempts to reduce transaction costs, thereby "repairing the inefficiencies" of markets via institutional policy (Spithoven et al 50). Douglas North (1991) describes institutions as "humanly devised constraints that structure political, economic, and social interaction. They consist of both informal constraints ... and formal rules. Throughout history, institutions have been devised by human beings to create order and reduce uncertainty in exchange" ("Institutions" 97). For North, one of the founding researchers of NIE, institutions evolve historically in consequence with their past, present, and future economies (97). NIE accepts neoclassical assumptions about scarcity and competition and tries to address them in light of institutional power and influence. Eva Lieberherr explains that NIE's methodology is diverse, and consists of a compilation of different theories and traditions such as economics, law, organization theory and industrial organization as well as drawing from political science, sociology, anthropology, psychology (cognitive science in general) and evolutionary biology ... in sum, NIE takes into account the profound effect of institutions on economic interactions as it acknowledges that legal, political, social and cultural institutions affect the performance of an economy. ("Policy Relevance of New 


\section{Institutional Economics” 2)}

In this section, I will discuss some of the major tenets of NIE, and how these theories relate to and help describe my objects of study.

While I do not think the goals of NIE are entirely relevant or possible for MMO markets, their assessment of market affordances and rule systems provides an interesting starting point to thinking about the relationships between institutions, individuals, and trade in online spaces. As demonstrated in the discussion of Castranova's work, scarcity and the supply and demand model are inadequate theories for describing virtual game economies. Nevertheless, I am interested in the descriptive value in NIE terminology, particularly the description of institutional processes or developments within MMO trading, from culture to design affordances.

John Drobak explains the centrality of institutions to NIE's economic theory as follows:

The new institutional economics takes its name from institutions: the rules that structure the economic, political, and social interactions in a society. Institutions can be formal, such as law, or informal, such as social norms. The new institutionalists believe that economic performance cannot be understood without paying attention to institutions. Consequently, their scholarship examines a multitude of economic and political issues by focusing on institutions. ("Law and New Institutional Economics” 1)

Moreover, NIE proponents argue that some of the assumptions made by neoclassical economics are inherently false. NIE rejects the neoclassical idea that humans act as rational maximizers of profit, use, or utility, and insists instead that institutional forces 
inevitably shape economies. Importantly, institutions are not always formal or bureaucratic structures, and are not always readily apparent or known. For example, certain institutions manifest themselves in norms or customs, while others appear in the form of governance that defines the formal rules of play within the market ("Policy Relevance of NIE" 6). From a game studies perspective, it is possible to view a game's rules and mechanics as institutions that govern play practices and decision making. Mechanics, as a later section of this paper will demonstrate, are rarely if ever economically or politically neutral. Under NIE theory, trade windows, auction houses, middle-men/women, the Steam market, and third-party auction websites are all institutional forces that shape player decisions.

While neoclassical economics purports the rational choice model for actors, Lieberherr explains that, under NIE, human actors are considered to have "bounded rationality rather than perfect knowledge" (“Policy Relevance of NIE" 4). NIE accepts that humans desire or imagine themselves to be making optimal decisions, but they are incapable of doing so for a variety of economic, social, or biological reasons. ${ }^{9}$ Moreover, NIE presumes human actors to operate with self-interest, following the assumptions of neoclassical economics, but diverts from neoclassical theory by stating that actors operate with uneven information, knowledge, or capacity. Actors in trading situations operate with asymmetrical preferences, as well, and all of these previous factors creates uncertainty within any attempted transaction. From a broader policy perspective, one of

9

Interestingly, Robert West and Matthew Rutledge-Taylor (2004) make a similar argument using a cognitive model about ideal gameplay that demonstrates a similar pattern. Game theory purports that individuals, at the optimal level, will play zero-sum games with random chance and logic. Their work, however, demonstrates that humans are incapable of playing that way, and instead prefer to metagame. That is to say, human beings base their decisions on previous information ("Cognitive modeling versus game theory"). 
the goals of NIE is to mitigate these uncertainties via institutions: "under the purview of transaction cost economics, the theory focuses on micro-analytic methods looking at institutional arrangements that sustain and safeguard transactions" ("Policy Relevance of NIE” 3). NIE theorists contend that institutional policy is a prerequisite for markets to function. ${ }^{10}$

Applying this to my objects of study, trade windows are most certainly an institution designed to reduce the uncertainty of transactions. Middle-men/women are as well, but come with a host of other social structures and risks that make them a less efficient institution. We might think of middle-men/women in The Realm as institutional forces negotiated by the community and given authority by the trade chat channel, itself another negotiated institution. Lieberherr explains that organizations occasionally form within institutions and markets to help manage and overcome perceived limits in human "cognitive abilities through coordination and cooperation" (9). MPs, the trade chat channel, guilds, and trade windows all operate as centralized efforts, opposite the previously mentioned decentralized coordination that constitutes markets. However, these organizations are institutional apparatuses designed to work in coordination with other institutions to enable market activity. As a result, the development of trade interfaces in games like Diablo, EverQuest, and World of Warcraft, apart from glitches and minor scams, successfully reduce the cost of exchange between players. Trust was

10

NIE complicates the neoclassical construction of markets. While neoclassical economics defines the market as the price mechanism's coordination of "transactions between minimally two parties, NIE defines it in more complex terms: a market is a mode of decentralized coordination employing high powered incentives to enable regular voluntary exchanges of property rights that are reversible and regulated by the price mechanism" (Policy Relevance of NIE" 9). Price mechanism refers to the cost of goods and their effect on supply and demand. Again, NIE maintains some neoclassical assumptions about markets that are not currently translatable to virtual economies, as Castranova and Lehdonvirta's independent findings on "mudflation" demonstrate. 
eliminated as a consideration among traders, for example, which saved time, thereby allowing players to spend more time pursuing other interests. Additionally, auction houses in World of Warcraft equalized the asymmetrical information and knowledge among players who may not know how much any given item ought to be worth.

Previously, players would manage this task in chat channel, but the auction house makes it much easier. The trade mechanics in World of Warcraft, then, alleviated what NIE theorists would describe as additional costs of transactions. Players could assume that trade interfaces, as an institutional affordance, will safeguard their transactions. Players would no longer require reference checks for middle-men/women, have to give first, to drop an item and hope to avoid being scammed, or engage in other precarious forms of trade.

Focusing specifically on objects of study in my dissertation, the obvious examples of interwoven institutional apparatuses are located in the Steam market and Stephenson's Reamde. Steam uses the national currency of its players, and converts this money into their Steam wallet. As a result, players can buy skins, trade, and sell items with only a marginal percentage going to Steam. Steam combines the affordances of two separate institutions: a fixed medium of exchange (national currency) with the alacrity of a video game auction house. As a result, trades and purchases are managed instantaneously with regards to currency exchange. Stephenson's virtual world of T'Rain in Reamde is the predecessor of the Steam market, still concerned with spatializing the virtual in order to accumulate capital, but mimetic and encompassing of virtual transactions. T'Rain combines various cultural and socioeconomic histories and institutions while accumulating the national currency of its users. 
Moreover, NIE endeavors to explain historical transformations in economies by analyzing their institutional influences. Lieberherr notes that "NIE holds that the diverse performance of economies can be explained by understanding the process of economic change. In turn, it is possible to comprehend this process through assessing institutions that can be defined as rules diminishing uncertainty in economic exchanges" (3). These two sentences succinctly describe the crux of NIE's relevance to this project. With the growing certainty enabled by auction houses and trade affordances, new economic possibilities began to emerge in the virtual world of MMOs. As players gained institutional insurance that their goods could be safely and efficiently delivered, realmoney trading increased and gold-farming entered the public consciousness, redefining the landscape of numerous MMOs.

Lehdonvirta notes that isolated cases of real-money trading for digital items occurred in the late 1980s involving MUDs, but not until the safeguarded trade practices in games like EverQuest do real-money trades increase in number (Virtual Consumption 42). While hard data on the amount of real-money trading happening in the early-to-mid 2000s is difficult to find, there are other indications that point to increased real-money transactions for virtual goods. For example, Castranova is able to collect data to estimate a GDP based on real-money exchanges on various third-party websites in the early 2000 s, and he indicates a substantial amount of trading in this sector. However, Castranova was an early adopter of virtual economic research and therefore a bit ahead of the curve regarding the emerging real-money transaction market. Perhaps more telling is Lehdonvirta's description of several countries, including South Korea and Finland, which passed laws in the early-to-mid 2000s regarding real-money transactions for virtual goods 
(7). Following NIE theory, these institutional metamorphoses indicated an economic change, and, more importantly, a change partially enabled by interface affordances within MMOs operating as institutions for reducing transaction costs. NIE, if anything, can help identify the tangible, legal, and economic relationships at play between institutions and markets. The rise of esports and skin gambling in the Steam Market has similarly resulted in new institutional formations, evidenced by various government interventions and regulations.

However, like its neoclassical economic ancestor, there are issues for which NIE cannot adequately account. Efficiency is a central component of NIE theory, and by efficiency NIE theorists refer to transactions that have the least amount of atrophy, so to speak. Those transactions by which people easily and optimally make informed decisions about their exchange are defined as efficient. Their view of efficiency is, essentially, a convoluted description of the neoclassical assumption about the market. Even two theoretical proponents, Eric Brousseau and Jean-Michel Glachant (2008), admit that the process by which an institution is created may in and of itself fail to guarantee efficiency, and managing institutions may be itself an inefficient process ( $\mathrm{New}$ Institutional Economics: A Guidebook 22). Nevertheless, O. E. Williamson (2000) contends that efficiency is itself a relative and precarious concept, and the ideal institution is mutable and changing ("The New Institutional Economics: Taking Stock, Looking Ahead" 612). As Lieberherr explains, "this reasoning is based on the theory's premise that human actors in a dynamically evolving world with bounded rationality can neither devise nor choose completely efficient institutional arrangements" (11). In other words, while a video game's trade interface may be designed with efficiency in mind, 
secondary factors, including other institutions, can prevent or hinder its successful implementation.

Either way, this project is not only interested in NIE's economic prescriptions or policy, but in its terminology and method of thinking about economic transaction in light of trade mechanics and interfaces. NIE theory provides a useful sociological tool for thinking about the linkages between interfaces and trade relationships in MMOs. As Lieberherr elegantly summarizes, "NIE is also regarded as enabling analysis of economic relationships in a multitude of social settings as it focuses on the fundamental premises needed for exchange: the creation and enforcement of the institution of rights (decision, use and access) that provides means for actors to coordinate in order to exchange resources" (17). Specifically, NIE highlights the manner in which minor institutional changes (the development of the auction house in World of Warcraft, for example) are often interrelated (legal adaptations), and can produce large-scale economic changes within a broader global context (real-money transactions, gold-farming, and third party auction sites). It bears mentioning again that trade interfaces, while seemingly innocuous, are actually an extremely powerful and influential institution.

Notably, NIE theory might accurately describe World of Warcraft's ban on realmoney transactions, which aimed to prevent the sale of items and exchange of accounts. Various secondary market sites for selling contraband items and accounts developed as a result of the ban. The creation of these institutions are an attempt to reduce the transaction costs of would-be real-money traders. Strangely, Blizzard has, in many ways, always been at odds with the concept of secondary markets in their games, apart from Diablo II. Even Hearthstone, Blizzard's digital collectible trading card game, does not 
allow players to trade cards between accounts. In part, this restriction is related to their desire as a game publisher to present consistently populated games with players "actually playing," as opposed to grinding out items for real-money exchange. Additionally, WoW was central to the player debates regarding achievement hierarchy mentioned earlier. Unlike The Realm before it and the Steam market after it, World of Warcraft seems stuck in limbo with regards to its economic identity. On one hand, Blizzard's insistence on keeping items and accounts part of a singular identity or diegetic role-playing experience hearkens back to early MMOs, whose players regarded their level and items as signs of mastery of the game (Lehdonvirta 47). On the other hand, World of Warcraft provides the necessary tools, via auction houses, scripts, trade-windows, and in-game delivery, to safely reduce the transaction costs of selling in-game items for real-world money. Yet, perhaps as a cultural holdout, Blizzard is dedicated to keeping the game from becoming an economic donnybrook like the Steam market. One of the issues with NIE becomes apparent in this very interaction: reducing transaction costs is in itself a subjective and malleable process. Reduction for one particular group might result in complication in other market sectors. In any case, NIE's argument that individuals attain information, knowledge, and ability asymmetrically ought to apply to institutions as well. Various institutional forces in WoW appear to compete with one another to manage trade costs (real money versus between established hierarchy players).

In the Steam Market and WoW, it is apparent that institutions often come into conflict with one another. The in-game trade affordances, for example, reduce the risk of players exchanging items with one another. As previously mentioned, security features are one of the Steam Market's primary concerns. Auction houses in WoW, additionally, 
eliminate the risk and time players need to spend acquiring items through player-toplayer trade. These affordances, therefore, reduce some of the transaction costs associated with trade, and thereby represent a successful institution according to NIE thinking. According to North, successful institutions are varied and manifest according to their relative historic location and requirements. North describes various institutions that allowed for historic trade routes using ship or caravan to operate successfully: "negotiation and enforcement in alien parts of the world entailed typically the development of standardized weights and measures, units of account, a medium of exchange, notaries, consuls, merchant law courts, and enclaves of foreign merchants protected by foreign princes in return for revenue" ("Institutions" 100). According to North, these institutions successfully lowered information costs and provided incentives for agents to interact peacefully and legally within the market. However, I have also demonstrated the manner in which in-game and real-world economies produce separate and competing transaction costs. Limitations on the Steam Wallet or EULA policies regarding account or real-money trading, for example, result in the creation of new institutions. Third party websites, such as the previously mentioned EpicToon, for example, exist for users to easily trade their in-game currency for real-world currency. These third party websites are also institutions which reduce a transaction cost by providing secure and effective methods for players to trade. These new institutions work to reduce the transaction costs of an exchange outside the purview of these in-game economies.

My major critique, however, is NIE's reliance and naturalization of various liberal ideologies, particularly about private property, individual freedom, transaction costs, and 
human desire. First, transaction costs are not necessarily obvious. Moreover, the transaction of goods and services can result in additional social transactions and exchanges may or may not benefit the traders. Marcel Mauss (1925), writing about gift economies, identifies the various ways that exchanges produce social and cultural capital, for example. Mauss identifies gift economies as a performative, visible form of a market (The Gift 4). As a result, these markets reveal the complex social life that is inextricably linked with the economy. To identify which relationships constitute transaction costs under NIE principles, then, seems pointless and difficult. Furthermore, a reliance on firm property rights is antithetical to the overall Marxist current underpinning this dissertation. The theory attempts to acknowledge human actor's freedoms with regards to an institution, but fails to account for who is "simultaneously constrained by it as well as engaged in shaping" (Lieberherr 20). More importantly, Lieberherr notes that "NIE lacks a single established definition of the concept of an institution," but assumes that institutions will operate as "systems of rules created to offset uncertainty and risk by providing a social structure that allows humans to gain control over their environment" (5) There is a paradox at play here in the assertion that self-interest governs individual trade patterns but institutions are fairly negotiated and constructed, especially considering NIE theorists' assumption that knowledge and information are unevenly distributed. Viewed as an institution, trade-windows ostensibly work to alleviate trade costs, but fail to account for the uneven distribution and market knowledge at play, resulting in the legal changes enacted in countries like South Korea and Finland. Additionally, neoclassical and NIE economics raises questions about the state of the individual within rule structures that I will address in light of Miguel Sicart's work on games and ethics. 


\subsection{The Influence of Interface and the Role of the Player}

This section addresses the principles that have influenced contemporary interface design, and reflects upon a variety of research that perceives interfaces as rule systems that work to create social effects. Additionally, this section will explore various methods of discussing games as a set of mechanics and unambiguous formal systems, while simultaneously addressing their necessary interaction with active, participatory human agents.

\subsubsection{Interface as Rhetoric.}

Thinking through the social operations of interfaces can be a difficult process, so perhaps it is best to begin with a pedestrian example that will help think through more gamic interfaces. A set of stairs, for example, can be imagined as an interface. It mediates between spaces, interacts with and enables users to perform a task. Moreover, a set of stairs makes an argument about itself and its capabilities, but also about the capabilities of its potential users. Like most tools, stairs provide a means to an end. Stairs also have a rule set, so to speak. Stairs allow one to travel up or down at varying speeds throughout a building. Sometimes stairs are an object for exercise and health. But, stairs also require legs or other physical ability to navigate. As Burdick et. al explain, "tools are not just tools. They are cognitive interfaces that presuppose forms of mental and physical discipline and organization. By scripting an action, they produce and transmit knowledge, and, in turn, model a world" (Digital Humanities 105). Stairs are built for 
users physically capable of using them and with a particular goal of moving throughout a building. They indicate that these buildings are designed for people physically capable of navigating them. My use of the term tool in this case can be interchanged with interface, which Ben Griffin and Laurel Baston define as "an aggregate of means by which people (the users) interact with a particular machine, device, computer program or other complex tool (the system)" (“Interfaces” 1).

Interfaces, then, reveal information about their users and indicate patterns of culture and social life. Burdick et al. contend that "it is important to be able to identify issues of access, inclusion, and power that interfaces and platforms reinforce or perhaps problematize" (vii). Recalling the earlier discussion of NIE, trade windows can be viewed as an institutional interface for minimizing the time it takes to trade. However, minimizing the time it takes to trade produces new and unforeseen cultural and social practices; one example of these practices was demonstrated in the NIE discussion surrounding the legal and economic institutions that arose to handle these new and efficient trade systems. As Burdick et al. explain, interfaces are "the embodiment of a project's argument and methodology" (14). This dissertation is concerned with tracing the sites of inclusion, access, and power for which trade interfaces make an argument. Interfaces such as trade windows, for example, make arguments about social life, about trading, about how items ought to be managed, and the efficiency of these processes.

Interfaces in the digital era have been designed with disparate philosophies and with various levels of concern about their social and cultural implications. Nevertheless, efficiency and usability have remained central goals. Mary Keeler and Susan Denning provide a brief summary of the nascent development of computer interface design's 
theoretical implications and justifications:

In the beginning, computers explicitly restricted their users to binary expressions and the idea of an interface between [users and expressions] had yet to be conceived. As the computer developed from calculator to general purpose symbol manipulator, the notion occurred that these machines could become extensions of each individual's information processing capability to increase our personal capacity for 'storing and retrieving information'. This laid the foundation in interface philosophy for assuming that an information theoretic approach could adequately account for human communication. When this notion became tangible in the first personal computers on our desks, some designers began to realize that the full potential for extension would be harnessed only when computer technology could provide conditions at the interface that more closely resemble those of human communication. ("Interface Design for Communication Theory" 285)

Early designers imagined escalators and not elevators in their search to improve stairs. However, according to Keeler and Denning, advances in computer engineering created a shift from computers operating as tools performing specific and purposeful tasks, towards hypothetical production spaces through which human interaction manifests. As a result, designers began to search for methodological guidelines for graphically depicting the computer's potential for information storage and exchange. Ultimately, interface designers settled on skeuomorphs ${ }^{11}$ as a method of harnessing the multitudinous algorithms and communication data computers were capable of performing. Personal 
desktops and operating systems composed of framed windows and filing systems resembling analogue office equipment are the result of this metaphor-based design structure.

Of course, the design of the desktop is not inherently a metaphor, but, as J.L Altya and others point out, an "analogical or model based approach" that encourages "the direct transferral of existing knowledge to a novel situation. In contrast, metaphors do not make explicit the relationship between metaphor and functionality" ("A framework for engineering metaphor at the user interface" 14). The metaphor comes to life at the level of user interaction. There is an active construction that takes place when users apply their previous knowledge of actual desktops to this "novel situation," thus drawing upon their knowledge of distinct items to process information. Foley and others note that "the design and selection of interaction techniques for a task must take into account the perceptual, cognitive, and motor processes involved, even when these seem to be trivial. In general, the design goal is to minimize the time taken, although this cannot always be measured in the case of cognition" (Interactive Computer Graphics 18). In short, the power of any single metaphor-based design is located within human-computer interaction, not simply within the physical manifestation of a system's design.

As Keeler and Denning note, interface designers began to concern themselves with satisfying individual needs as well as enabling social interactions. Digital interfaces determine cultural formations through a diffusion and exchange of networked information. Interfaces in the digital world must often account for their involvement in social media, participatory game play, and information production. As Burdick et al. explain, "understanding the way one structures the relationships among data, the ways in 
which users input and access information, and the physical and conceptual design of such systems" is central to understanding contemporary interfaces (Digital Humanities 20). Issues of access and information management are nothing new to interface design, as indexes, table of contents, library stacks, and other pre-digital interface management systems indicate. However, the digital interfaces under discussion in this dissertation tend to produce value through their use at a level of abstraction and production unrivalled in the pre-digital design world.

\subsubsection{Video Game Mechanics and Interfaces}

Moving the discussion from interfaces generally to video games specifically, mechanics, rules and interfaces are often discussed holistically by game designers and theorists alike. Mimicking the model of early computer interface design, game mechanics similarly seek efficient communication and interactivity with players by communicating the possibilities and affordances of each contextual game object, mechanic, or graphic interface. Carlo Fabricatore summarizes interaction between players and mechanics in simple terms: "when playing a game, players seek challenge, mastery and reward, all packed in entailing and motivating activities. From this stems the importance of game play as a crucial game design cornerstone, and game mechanics as tools that the player has to interact with in order to carry out game play activities" (“Gameplay and Game Mechanics Design" 1). The difference between non-gamic user interfaces, such as a music player or Google maps, and a video game is partially found at the degree of interactivity. The play experience, according to Fabricatore, is "centred on 
a decision-making process that relies on the information conveyed to the player.

Information is transmitted through visual (graphics and text), aural and even tactile means" (1). Fabricatore separates these types of interaction into two distinct categories: functional and aesthetic. He explains that functional information allows players to complete the basic functions or mechanics in order to play the game, while "aesthetic information defines most aspects of the context in which the game takes place, and is mainly aimed at rendering an atmosphere capable of drawing and maintaining players' attention on an emotive basis, making them feel part of an entailing virtual world" (1). Gordon Calleja's In-Game (2011) similarly imagines players and games coming to life at the level of information exchange, thereby prioritizing interactivity as a communicative feedback loop coordinated between the player and the game. By coming to life, I mean the manner in which players can be said to inhabit the space of a game. Calleja describes presence, similar to Marvin Minsky's telepresence (1980), as the "combination of the operator's actions and the subsequent video, audio, and haptic feedback" of the game (18).

Of note, most game designers and game design researchers discussed in this dissertation, such as Zimmerman, Salen, and Fabricatore, tend to construct the player as a more active participant in negotiating play than game studies scholars from the humanities (Alexander Galloway, McKenzie Wark, Nick Dyer-Witherford). It will become apparent, for example, that many humanities critics, particularly on the political left, account primarily for the "text" of the game, so to speak. However, the two are not incompatible schools of thought. Game designers, as Eric Zimmerman notes, are more concerned with developing tools and play patterns than they are assessing the 
sociological or literary outcomes of games ("Jerked Around" 2). The reason for game designers' lack of concern regarding interpretive qualities, Ian Bogost explains, is that game designers typically approach games from a perspective of code and mechanics, and are therefore concerned with fundamental programming, human-computer interaction and usability, and player enjoyment ("Video Games are a Mess" n. pag.). For example, the spaces of play described by Huizinga, Caillois, and Suits that I mention earlier in this dissertation lead Katie Salen and Eric Zimmerman to develop a much-maligned term called the magic circle (Rules of Play 1999). For them, the magic circle is a nuanced idea that hopes to explain the different relationships and meanings that develop during game play. Zimmerman explains broadly that "games are a context from which meaning can emerge" ("Jerked Around" 2). Zimmerman explains that after their work suffered through a decade of straw-man arguments by game studies critics, he was forced to explain and defend the use of the term magic circle. He felt the need to respond to the consistent backlash among academics who mistook the use of the term as a formalist/structuralist obsession with rules. Salen and Zimmerman do not argue that rules and structural limits wholly or exceptionally dictate the shape of play regardless of any personal, economic, or social contexts. ${ }^{12}$

Alternatively, game studies in the humanities is often, according to Bogost, more concerned with form and function, which "focuses on the operation and behavior of the

12 Zimmerman provides an excellent analogy of a chessboard in his living room. He notes that a chessboard on a coffee table might create various social meanings. It is possible a guest may assume he enjoys playing games. Or, perhaps the guest assumes it is a decorative piece. Or both. While talking before a game, perhaps they fiddle with the pieces. But when the game starts, there is an agreement to disregard casual fiddling and behaviours in favour of rules. Perhaps the conversation lingers as the game continues, but their personalities, conversation, desires, and focus are altered by the mechanics and rules they have now agreed to play by. 
program. This is where we find approaches to the operation of the program" (n. pag.) ${ }^{13}$.

Bogost expresses a design philosophy similar to Zimmerman, for example, that accounts for both code and player experience:

The rules do not merely create the experience of play-they also construct the meaning of the game. That is to say, the gestures, experiences, and interactions a game's rules allow (and disallow) make up the game's significance. Video games represent processes in the material world - war, urban planning, sports, and so forth — and create new possibility spaces for exploring those topics. That representation is composed of the rules themselves. We encounter the meaning of games by exploring their possibility spaces. And we explore their possibility spaces through play. ("The Rhetoric of Video Games"121)

In fact, the formal and functional elements that define the structural limits of a game are the qualities that enable play, according to these designers and theorists. I tend to agree, although I believe that the majority of users operate, at least as far as trade practices are concerned, within a high degree of technical and functional constraint. I address the issue of constraint and rule structures in relation to players in more detail later in Chapter 2, but I contend that a game with no trade affordances provides a more robust space in which to interact and play from a game design perspective. If trade moves from an inhabitable space to a functional process like, for example, the shift from middle-men/women to

13 In this article, "Video Games are a Mess," Bogost also provides other possible spaces of exploring video games as objects, from platform studies to reception and operation, which he refers to as "focuses." The point of his article is that there is no singular methodology to explore video games. Moreover, Bogost contends that both Ludology and Narratology, two classic and competing approaches to game studies, exist within the same purview of form and function, each concerned with defining the discernible or executable operations of a game (its user interfaces, narrative, storylines, NPCs, characters, et cetera). This dissertation is largely concerned with the operational outcomes of form and function, as well as interface reception, and less so with reception, code, or platform. 
trade windows, the space for possible playful interactions is reduced. The relevant point here is that game designers and scholars are not mutually exclusive. Moreover, it is both necessary and pragmatic to account for the practical design and theory which models contemporary mechanics and game design in order to understand how it expresses itself operationally.

Many game studies scholars have studied the influence of mechanics and rule sets on player learning or satisfaction. For example, multiple critics have written about games in terms of puzzles and reward systems. Espen Aarseth writes about the feeling of aporia $^{14}$ in completing particularly difficult tasks in games, while Jesper Juul argues that failure (to make the jump with Mario, for example) eventually guides players to learn the system and behavioural patterns necessary to successfully navigate a game world. Broadly speaking, mechanics provide a set system from which players can derive lessons, methodologies, and ideologies. This propensity for games to teach players new interactions and systems is partly responsible for the onslaught of writing about education and games over the last few decades. Soren Johnson, writing in the collection of essays Games, Learning, and Society, argues that mechanics give rise to meaning and not theme. Speaking about World of Warcraft, Johnson explains that the game may have a swords-and-sorcery theme, but the mechanics encourage the players to conduct their own form of natural selection when deciding how to develop their characters ... O Over years of experience, veterans of WoW have established a number of upgrade paths (or builds) for each class depending on what role the player wants the character to fill ....These paths have evolved organically over

14 Aarseth uses aporia as a term for confusion or puzzlement that is resolved by an epiphany (Cybertext 2). Specifically, Aarseth uses the term to indicate choice on behalf of the player or reader. He refers to an aporia as "the absence of a possibility" from choices unmade and paths untaken (3). 
the years as players tried out different combinations depending on what the game rewarded or punished. ("Theme is not Meaning" 34)

For these theorists, game play emerges at the intersection between mechanics or designed structures and sociocultural interactions. Grand Theft Auto, for example, is not necessarily about acquiring wealth or running over prostitutes with stolen cars. Kurt Squire, writing about Civilization IV, explains that the game provides a grammar or lexicon with which players can interpret histories and cultures ("Designed Cultures" 11). Applying these theories of learning systems to a political landscape, Michael Burden and Sean Gouglas argue that as "algorithms are used in and applied to social situations they become forces that shape and persuade. Bureaucracy is just one well-known example of the tyranny of ceding control to Kafkaesque algorithmic processes" ("The Algorithmic Experience: Portal as Art” n. pag.).

Instead of interpreting the rule sets, other scholars of MMOs have studied how game mechanics contribute to the social network and cultural practice of gaming communities. It is worth noting that rules and mechanics require player effort, desire to play, and cooperation in order to produce a successful (populated) MMO. Within MMOs, these emergent systems can be located in game play moments such as competitive metagames, or in user-interface interactions which form a complex system of trade practices. Pearce's research primarily investigates how the "design affordances of these worlds lay the groundwork for emergent behavior ... [and attempt to] reveal the ways in which constraints and affordances, as dictated by the world's designers, serve as the raw materials for large-scale emergent behavior" (36). Pearce's ethnographic work, however, was produced at a time in which MMO culture was, by and large, less 
prescriptive and item-driven than it is today. The game Pearce documents, Uru: Ages Beyond Myst, and its associated diasporic community, was less concerned with economic activity than many contemporary MMOs, at least in her description. More importantly, new ethnographic work must now account for RMTs (real-money transactions), and their massive influence on in-game economies. Her work, I believe, would be difficult to repeat so easily today, as migrant groups are probably extremely small, and emergent behaviour is so wildly dominated by economic desire. Thomas Malaby (2009) has similarly investigated the influence of design mechanics on cultural practices, for example, but importantly documents the effect of commodities in Second Life, and demonstrates the power of these virtual items to tie communities and players to a particular virtual world.

\subsubsection{Players, Ethics, and Interface}

Writing about EverQuest, Greg Lastowka emphasizes that its narrative world of Norrath, "like a book or a film, does indeed present a text that can be divorced from its players" ("Planes of Power" n. pag.). The diegetic world of the game contains a rule structure, code, animations, narratives, and characters that can be approached, however inadequately, from a formal literary perspective. According to Lastowka, the virtual worlds of video games can be broken down into three areas of study: the pre-player text, the single-player text, and the multiplayer or community-driven text. Studying the preplayer text of a virtual world involves analyzing the core elements of the program, the mapping of the world, classes of characters, skill and job systems, without interruption 
via user input. While a significant portion of this dissertation examines pre-player texts and their ideological implications, other portions are concerned with community reactions and negotiations with these texts. As such, it is important to construct a method for understanding how player experience might be discussed and examined. In order to construct a player model, I draw upon Sicart's examination of ethics, discussed in this section, as well as Autonomist Marxism's imagination of the socialised player, discussed later.

One method of analyzing the trade affordances and practices in relation to players is by employing Miguel Sicart's construction of video games as an ethical object. Sicart's work combines value ethics and informational ethics to construct a vision of cooperation between player and game that he describes as ethical. Sicart describes ethics as a system or set of moral values, and the tools for analyzing these values. Morals can be defined as the right or wrong of actions or objects. The application of ethics is the rational, philosophical approach to the questions of good, evil, harm, duties, and values. ... Roughly stated, virtue ethics attempts to define the ethical virtues that human beings and human communities should aspire to exercise in order to be ethically sound. Virtue ethics is an ethical theory about the practice and development of the moral characteristics and practices that make human beings moral animals who aspire to the good. (The Ethics of Computer Games $13,110)$

First, I should clarify that Sicart is not attempting to essentially moralize games in any way. Instead, he is attempting to negotiate the ability for players to display agency, and describe what that agency might look like. He rejects the idea that players are "moral 
zombies, unable to critically reflect on what they do inside or outside the game" (201).

He insists that ethical games are determined by their ability for player decisions to shape the game world. ${ }^{15}$

How Sicart defines user experience in relation to rule systems is critical to understanding his construction of video game ethics. Although Sicart argues that video games carry inherent structural power, he also believes that player interaction is paramount to ethical game play. Video games, he explains, can be considered moral objects if "ethical discourses and values can be found embedded in the practices suggested by the rules and take place in the space of possibility. If the space of possibility of a computer game can be analyzed using the tools of ethics, and if that analysis is corroborated by actual game play, then we can say that a specific computer game is a moral object" (51). It might be helpful to imagine Sicart's ethics as a negotiation between formal structures and player desire. Furthermore, Sicart insists that any game's ethical relevance is determined by its referential or allegorical relationship to problems in the real world (57).

Sicart's definition of video games combines two ontological perspectives: form/function and interface operations. Bogost defines the study of form/function as focusing on approaches to "the operation and behaviour of the program," and interface study as a method of addressing a "user's relationship to the visible, operable part of a computer system, including the discipline of human-computer interaction, visual, filmic, and art historical approaches." One reason I categorize Sicart's work this way is because

Sicart makes distinction between a game and its world: a game world "is either the simulation of the material conditions of a game, like in the case of computer-simulated board games, or a simulation of another world. That simulation presents both simulation rules and game rules: in fact, virtual environments are constrained by the game rules, since all the elements that are not fundamental to the game are a mere setting for the actions of the game" (34). 
he heavily relies on early game studies scholars, such as Espen Aarseth and Jesper Juul, to define video games, and this sampling shapes much of his argument. For example, Juul (2003) defines a game as "a rule-based system with a variable and quantifiable outcome, where different outcomes are assigned different values, the player exerts effort in order to influence the outcome, the player feels attached to the outcome, and the consequences of the activity are optional and negotiable" ("The Game, the Player, the World" n. pag.). Juul's early work was embroiled in the Ludology versus Narratology debate, which pitted formal rule structures against narrative or player experience as primary sites of study. ${ }^{16}$ Following the death or at least decline of these debates, Juul identified a new debate regarding a game-centric versus player-centric model of thinking about games ("Zero-Player Games" n. pag.). He argues that games ought to be thought of as experiential objects whose formal rule structures are given life by players' "personal and play contexts." Note the similarities between Juul's description and Sicart's definition of a game: "Most computer games are systems of rules that encourage players to work toward goals in a virtual environment. And many computer games address players by means of a story. There are, then, two fundamental elements to these computer games: systems and worlds" (The Ethics of Computer Games 21). Sicart, however, clarifies that visual, representative, and narrative elements are secondary concerns when addressing the ethics of computer games (22).

Although he acknowledges the necessity of players in order to enact a moral procedure within a video game, Sicart admits the power of the formal properties of interface, mechanics, and rule structures similar to the design theorists above (Burdick et.

16 The introduction of Stephen E. Jones's The Meaning of Video Games (2008) provides an excellent summary of the history of these debates. 
al, Keeler and Denning, Salen and Zimmerman). For Sicart, a "computer game's morals rest in its design," and the "systemic core of computer games, their rules, is of fundamental importance in understanding the ethics of games" (24). Interface operations, like trade windows, auction houses, and in-game mail systems, provide the (mostly) immutable structures that determine the shape of the game world and playerbase's behaviour. I draw similar conclusions throughout this paper, and presume, for reasons I will address shortly, the player-base is often incapable of making knowledgeable and therefore ethical decisions regarding various trading practices that date back to the mid-to-early 2000s. Additionally, Sicart is keen to point out the valueladen formal structures which govern the potential for particular play patterns to emerge among a user-base.

Rule structures, for Sicart, are in a power dynamic with a game's player-base. In fact, Sicart constructs players as subjectivities formed in the process of voluntarily playing a game. He explains his reasoning as follows: "computer games are power structures. Power creates subjects, and so games create players. The process of experiencing a game and becoming a player needs to take into account how the nature of the game contributes to the creation of that subjectivity" (68). In order to support his argument about the player-subject, Sicart draws on Foucault's re-imagining of power as an operative social force. Instead of being controlled, managed, and isolated, Foucault theorizes power as a generative operation that exists within relationships among subjectivities. The act of playing a video game is a form of knowledge production and therefore produces subjects, according to Sicart's reading of Foucault. Sicart explains that the "process of experiencing a game as a player-subject takes place in 
interpretational layers that provide different yet complementary ontological feedback to the process of becoming a player. It begins with an ontological move voluntarily made by the player: accepting the game as object and its conditions for success" (87).

He explains that "computer games seem to obscure and impose the rules due to their digital nature, but players are still empowered when playing a game, and the game experience is always under the sign of those rules that are not written, but that tell us how to play the game" (36). Sicart's use of the word obscure refers to something called "black box syndrome," which "describes how digital technology applied to computer games obscures the actual presence of a system of rules that determines the victory conditions and the inner workings of the system. By not showing how the games' rules are enforced, digital games tend to strengthen the supremacy of the rules system in the experience of the game" (27). Sicart notes that numerous examples exist of players attempting to fully comprehend the rule system in order to increase their play skill.

All this is a bit abstract, but it essentially results in a relationship in which players agree to play according to a set of rules, and negotiate their own goals and desires in accordance with the community of other player-subjects and rule structures of the game. The ethical relationship between video games and players that Sicart imagines takes place at the nexus of player-subject desires and the expressive power of rule systems. Importantly, Sicart explains that a player-subject ought to be conscious of the power structures that define the game (89).

From a trade perspective, ethical practices have become increasingly difficult among MMOs. The scope and power of video game economies has risen drastically and become globally significant and massively monetized. The information is scarce, but the 
pieces of data available on trade relationships in MMOs paint a picture of uneven wealth distribution and a community of players struggling to grasp its implications. Foucault's construction of power as a generative and networked source of knowledge is problematic in light of, for example, the Steam market's consolidation. Sicart, however, does identify World of Warcraft as an unethical game for its inability to provide players with meaningful community intervention (185). He notes the ethical qualities of EVE Online, a game with a vocal community prone to protest, because developers have allowed their player base to contribute to the values they see as relevant (188).

The example Sicart uses to demonstrate if an interaction between players and mechanics is ethical is World of Warcraft player-versus-player ( $\mathrm{PvP})$ combat system. Initially, PvP was permissible on certain servers which were marked as spaces intended for such combat. There were no rules governing combat other than geographical ones. If, out in the wilderness, a player saw an enemy from an opposing faction, they could attack them. As World of Warcraft's story is about two groups, alliance and horde, in a bitter, and never ending war with one another, some saw PvP servers, and PvP actions that took place in contested areas, as a diegetic element of the game. Sicart recalls that $\mathrm{PvP}$ occurred infrequently in low-level areas, but was common among higher level characters who viewed PvP as a sort of end game or means of experiencing new content. Despite no formal rule system as "a general and not always respected rule of sportsmanship, high-level characters would spare the life of lower, less powerful players" (182).

This all changed, however, when the honour system was introduced by developers. Players would receive rewards for points based on honourable kills, which 
were determined by a gap of 8 levels between each fighting player. A level 8 versus a 16, for example, would reward points. Additionally, these points could be used to purchase useful, powerful, and expensive items. The honour system had no punishment, however, for jumping lower level characters, and instances of deviant behaviour like corpse camping for respawns increased. ${ }^{17}$ The reward system encouraged players to adopt aggressive play patterns. The community, Sicart notes, was thrown into chaos. Some players complained it unbalanced the game, while others complained of harassment. There were, however, some players who enjoyed the competitive nature of the new honour system. Eventually, developers responded to player feedback and introduced a feature called "battlegrounds," an extra-diegetic space in which players could voluntarily travel to engage in PvP. Sicart points to the divisive reaction among the community as evidence of players' active participation in constructing game worlds. It is not reasonable to view players as "mindless subordinates to the game system" because multiple variegated responses to the honour system were made (184). Sicart contends that the honour system failed to provide an ethical framework, and thus resulted in community backlash. He explains that any ethical issues a game creates are only player concerns if game designers allow space for players to construct their own values.

One notable difference between the situations Sicart describes in World of Warcraft and The Realm is the management of player death. Most of the mechanical features are the same in a lot of MMOs. Dying in The Realm, as in many other games, gave a player the option to respawn at the location of death in fifteen seconds or to safely teleport home. However, a player who remained in the location they died might simply

17 Camping is when a player finds an advantageous spot and sets up camp, never moving around the rest of the map, while picking off other players. 
respawn to die again, either from monsters or other players that killed them. The corpse camping in WoW happened in The Realm, as well. It was often better to teleport back to town and move on. Like WoW, The Realm's PvP system was optional, but once enabled stayed on for a month, allowing any character of any level to attack another player in neutral territory. The major distinction, however, was that dying cost players both experience (and thus potentially levels) and items. Random items from a player's inventory were dropped upon death, so PvP was a game of risk and reward. In any case, those willing to engage in PvP knew the rules and voluntarily engaged, aware of the risk. There is an interesting ethical situation using Sicart's description. The game's mechanics set forth an argument that items and possessions were expendable in the name of diegetic continuity. Similar to the initial PvP areas that Sicart discusses in $W o W$, players generally policed their own behavior despite the reward of other people's items.

In order to understand the relationships between agents, rule structures, players, and ethics, Sicart identifies four levels of abstraction:

1. the game as a system of rules, mechanics, challenges, and goals; that is, the world of Azeroth as a space for play.

2. the player as an in-game agent; that is, the player that selects a class and plays according to the affordances and constraints of that class, leveling up and completing the quests relative to that class.

3. the player in the world; that is, the player as how she relates to other players by means of the tools, challenges, and methods provided by the game.

4. the player as a homo poieticus; that is, the player in the community creating and actively enhancing values on the good play. (185-186) 
Sicart concludes that, in the third level of abstraction, players in the game world are encouraged to behave in socially disruptive ways that are irreconcilable with the fourth layer of abstraction. Sicart argues that, in the fourth abstraction, players "of this virtual world, and of every virtual world, effectively present active stewardship in the construction of the values and behaviors of that world, and they can do so from inside the game, if that is possible, or by participating in the game community" (186). It is unclear from Sicart's text if these abstractions are meant to be hierarchical, or if they are to operate simultaneously. For example, it is unclear whether complete harmony is required across all four abstractions for a game to be considered ethical. To be fair, if the first two abstractions find themselves at odds then the game is probably unplayable. However, I raise this question because it seems Sicart values the ability for players to engage or reach the fourth abstraction without any form of dissonance. Given his tendency to prioritize the ethical and conscious operations of players, and his insistent that games are only experienced through play, I interpret his four abstractions to be hierarchical.

Sicart's approach to information ethics foregrounds the player as the primary ontological figure in the act of play. Sicart describes information ethics as "macroethical approach, a framework that expands the responsibility of moral agents by defining existence as informational existence: we are all data entities. Every biological life is a data entity, but there are more data entities than life-forms: there are artificial data entities that need to be respected and that can be harmed" (128). Moreover, information ethics is an architectural construction of ethics that views media ecology as part of an infosphere. "The infosphere," explains Sicart, "is an ecological environment of informational agents, patients, and their mutual relations. All elements of the infosphere are in one way or 
another mutually connected, precisely like in an ecosystem, and the balance of this system can be affected, leading to harm and thus defining what unethical actions or relations are" (129). This philosophical construction of information ethics is rooted in object-oriented ontology, a philosophical approach championed by Graham Harman, Quentin Meillassoux, Levi Bryant and others. Within game studies specifically, Bogost has been a proponent of object-oriented ontology as a methodological approach to games. $^{18}$

However, I interpret Sicart's construction of the player as fundamentally at odds with object-oriented ontology. For example, Sicart insists that:

From a virtue ethics perspective, the player has to be understood as a virtuous being. When immersed in a game situation, players use their motor skills, their capacities for abstraction and logic, and their intelligence to solve the challenges posed by the game. And they also apply their ethical reasoning. This implies that players are responsible for their acts in computer games. Players have the moral responsibility of creating values in the experience of computer games; they are the ones who will create the experience that will make a game ethical or not beyond the limits and constraints of its design. The responsibility for the affordances in the design is still the developers', but players must be considered responsible for the game experience and for how that game experience creates values for the community. (116)

18 In his essay "The Poverty of Philosophy: Realism and Post-Fordism," Alexander Galloway is extremely critical of object-oriented ontology. He contends that proponents of object-oriented ontology must necessarily equate human life with pieces of trash. Moreover, he explains that this philosophy denies the gravity of human suffering and inequality under capitalism. Nevertheless, Sicart's construction of player agency does not necessarily fall in line with Galloway's critique of objectoriented ontology. Sicart, as mentioned, tends to hierarchically assign value in regards to agents within the infosphere, for example. 
Sicart prioritizes the hermeneutic experience of the player, not the objects. In this regard, Sicart is not as reliant on the infosphere's object-oriented ontology as he suggests. His construction of hermeneutics comes from Hans-Georg Gadamer, who stressed the experiential properties of hermeneutics. Sicart himself does not draw on this example, but Gadamer's exploration of the term "wound-read" is useful for thinking through Sicart's construction of player experience. In his book on Celan, Gadamer comments on Celan's use of the phrase "wound-read" (Gadamer on Celan). Gadamer argues that the poet transports something wound-read, as in what is painfully gathered and transported in general. ${ }^{19}$ For Gadamer, the wound is the implicit acknowledgement that human knowledge always and already consists within its own horizontal limitation, both by and for its very nature. The wound serves as a tacit force that reminds us that human knowledge is a fabric woven by two threads, experience and finitude. Gadamer, commenting on Celan's poem, explains:

the poem's real message is this: what surfaces is 'wound-read.' 'Wound-read,' or wound-driven, refers to a wound produced by a reading expedition that has lasted too long. Or is there a deeper ambiguity in 'wound-read'? Perhaps it refers not only to the pain of reading, excessive or futile reading, but also to the pain and the wound of what is 'gathered,' that is, what is painfully experienced in general, which can also mean 'gleaned': gathered together, as in a gleaning of suffering. . . . Either way, what has been transported into words, translated into words, is the poem, the text wrought out of the unconscious depths with help of a kind of dream-work. (107-108)

19

Celan only says "wound-read" once, and Gadamer's lines on it are brief, but it is one of those epiphanical moments in which a possible understanding of experiential ephemerality becomes available. 
To wound-read is to transport a reader back into the fullness of the time and place where the original instance was felt. To recognize that, say, a poem is wound-read is also to recognize that experiences are wrapped in space and time. Or, in this case, players are wrapped in rules, mechanics, communities, and game cultures. The wound attempts to prevent the fissure of experience from being widened into two different instances by the force of time, such as, for example, the instance of statement, and the instance of reflection. The reader re-opens the wound by engaging with the text, and the nature of the wound-read is to prevent the reader's transcendence. Hermeneutics is, for Gadamer, an orientation rather than a method. Once people give up on the failed project of achieving a terminally accurate, terminally oracular language for human affairs, they recognize that the intelligibility of human experience consists in its evocation. Therefore, the wound-read is the reproduction of that experience.

The act of play, the experience of play, is itself an interpretive process derived from the active creation of value by users. The ontological existence of a game is an immediate process that manifests only through player engagement. The wound-read, in this case, can be thought of as the agency of players in the game world. Their involvement is what allows Sicart to make an ethical argument about the experience of play. In the act of playing a game, users construct meaning and therefore virtue. The player and their experience, then, take priority over the text (in this case the game) in Sicart's construction of ethics. The rule structures and mechanics of a game are textual constructions that are experienced by human perception, and this perception is the determining feature of ethical game play. Sicart's Ludic Hermeneutic Circle, for example, filters all potential gamic moments through different iterations of player 
identities (122).

Of note, employing Sicart's ethical construction to The Realm's trade affordances can follow a similar ladder of abstraction. Following Sicart's example, in fact, we see that The Realm places no technical affordances or tools on the third abstraction with regards to trade. Instead, players are given stewardship over their own community and their decisions for how they want to engage in trade practices. The result, according to Sicart's logic, is an ethical trade practice. The lack of affordances, mechanics, or challenges provided by the game with regards to item exchange indicate that trade is an ethical act if granted final abstraction.

Turning this same approach towards trade interfaces in $W o W$, however, becomes increasingly complicated given the frequent of real-money transactions, gold-farming, and disparate server types dedicated to PvP, Player versus Environment (PvE), Role-play (RP), or RP-PvP, each filled with different types of players. It is difficult, however, for Sicart's ethics to fully account for the manner in which economic rule structures may thoroughly fail to incorporate swaths of the game's player-base. For example, "farming" resources is mentioned only once, and I am curious if his ethics support the Western gaming communities' rejection of such practices, and, importantly, on what grounds. Yee writes about the inherent inequalities and racial tensions surrounding "communal" rejections of gold farming for what are ostensibly ethical reasons (Daedalus Project 2006). From interviewing players, Yee relays that players indicate a feeling of resentment toward gold-farmers, whom they feel "ruin" the game by not playing in the same way as others. Honed by hours of grinding, farmers often become incredibly efficient at managing their play and timing respawns of monsters in order to maximize 
their value over time. At what level of abstraction can gold-farming, for example, be judged? From the first two abstractions, which state that games are systems of rules and players are agents within this system, gold-farmers abide by the mechanics (disregarding any EULA infractions, which might make this whole point moot), and operate on the same sort of level grind as others. Gold-farmers might be said to maximize the third abstraction as individuals fulfilling their individual desires by mastering the finer tools and challenges afforded by the game. The crux of this issue, I imagine, rests upon the fourth abstraction by which players operate as virtuous agents.

However, making a judgment here seems difficult. Although gold-farmers defy the broader community standards, are these standards themselves virtuous or good? Is playing a game for entertainment inherently an act of good play while playing as a form of labour is not? One option is to presume that player stewardship is a democratic process by which players collectively manage their goals and values. However, this presumption does not seem to line up with Sicart's overall construction of doing good. Answering these questions might require one more layer of abstraction that considers whether a player can operate fairly as a virtuous agent under unequal socioeconomic circumstances. From there it is possible to consider what bearing, if any, non-diegetic circumstances have on the construction of moral play.

Another option is to look towards the technical rules and affordances which permit gold-farming as a viable play strategy. Of course, I contend that trade interfaces, such as auction houses, trade windows, and mail systems that allow characters to accumulate massive amounts of virtual goods quickly and efficiently are at least partially responsible for this behaviour. If that is the case, however, then the ethics of the interface 
and game are also ethical concerns outside the game. The existence of gold-farming relies on the uneven exchange between currencies, after all, and, as Julian Dibbell notes, some of these farmers view their work as a respite from even worse physical working conditions ("The Life of the Chinese Goldfarmer"). Regardless, it is possible to speak to the unethical qualities that these trade affordances represent. As I demonstrate in the next chapter, technology can often be an indicator as opposed to an operator when it comes to ethical problems.

Sicart's work provides an excellent conceptual framework for thinking through the manner in which players can ethically negotiate their game play. Yet, Sicart seems keenly aware that MMOs in particular are extraordinarily difficult to predict or discuss, and instead we should look to specific events (of which he provides a great example regarding $W o W \mathrm{PvP}$ ) in order to determine the ethics of any particular game or gamic moment. Importantly, Sicart provides a method for thinking through the limitations players face when engaging with various rule sets and design structures. For example, he provides a method of thinking through the institutional paradox regarding Blizzard that I mentioned previously. Players and institutions might be viewed antagonistically in some aspects of game play. In the early MMOs previously discussed, the player base fought against developing trends resulting from in-game economies and succeeded in having developers restrict the sale of accounts and in-game items. Sicart correctly notes that, while the game rules, mechanics, and interfaces are regulatory in nature, it is possible to effectively judge the merits of a game on its ability to produce meaningful (ethical) interaction at the player level. 


\subsection{Marxisms and Historical Materialisms}

This section discusses Marxist terminology, cultural, political, and economic theory, as well as post-Fordist and post-Marxist constructions of contemporary networked social life. It is important to note that Marxism is incredibly diverse, and instead it might help to think, "in exemplary postmodern fashion, not so much of Marxism as of "the Marxisms"' (Cybermarx 8). Nick Dyer-Witherford notes that heterogeneity among Marxist philosophy is the result of Marx's own conflicting ideas and inconsistencies, resulting in interpretations of his work that "have been selected, permutated, and refracted into an array of very different, and sometimes fiercely antagonistic forms" (8). Similarly, Daniel Little notes the extensive influence of Marx throughout a variety of academic fields, from art to the social sciences. Marxism, then, has produced a sizable lexicon and assemblage of theories: "the theory of alienation, the labor theory of value, the theories of class conflict and exploitation, the theory of the forces and relations of production, or the theory of the mode of production. So the question of Marxist method is complicated" ("Marxism and Method" 1). Marxist critics describe video games from nearly all of these perspectives, and I outline some of those below. Additionally, I focus on the cultural and economic descriptions of social life that contribute to my understanding of video game trade interfaces.

This dissertation's reading of trade interfaces and practices in the games it analyzes is influenced by Marxist cultural critique and a Marxist economic standpoint. It contends that Marxist theory is necessary in order to understand the socioeconomic relevance of trade affordances. Additionally, this work borrows liberally from the 
terminology and ideas outlined by Sicart, Galloway, Burdick et al., and others in their attempts to describe the influential structural properties of interfaces. Much of this critique is derived from the assumption that game mechanics contain inherent arguments about the cultural practices of trade, private property, and economic individualism.

If anything, the Marxist method of inquiry tends to be predominantly materialist. For his part, Marx attempted to produce an empirical and observational description of the capitalist system, thereby illuminating the institutional structures which governed class relations and economics. Moreover, Marx attempted to position these observations within a historical framework in order to assess capitalism's potential futures. In his assessment of material conditions, Daniel Little explains that Marxism also tends to focus on the forces and relations of production, and it postulates that technology and power are fundamental with regard to other social formations (literature, culture, law) ... it is oriented to the salience of class and class conflict within historical change. It is sensitive to the workings of ideology and false consciousness in our understandings of the social institutions within which we live. And it pays special interest, and offers special concern, to the perspectives of the underclasses at any given time in history. (4)

This dissertation likewise attempts to trace an institutional structure's contribution to capitalism in the age of video games. In so doing, I will assess some of the class conflicts (beyond even gold-farming) and material institutions (trade windows and other trade interfaces) which govern video game and interface design practices.

\subsubsection{Technology, Labour, and Production}


Marx viewed technology, at least specifically under capitalism, as a tool for expanding markets and accumulating value. Communication and industrial technology worked simultaneously toward the "autonomisation of the world market" (Grundrisse 99). Amy Wending notes that Marx's later work came to view machinery "not simply as a product of the division of labor or the related expansion of human knowledge and material wealth but historically, under the influence of market forces" (Karl Marx on Technology and Alienation 68). What technology can help describe, then, is the market conditions under which divisions of labour and inequality operate. By examining the "material" institution of trade interfaces, for example, it is possible to note class divisions among players. In particular, recall from the last chapter the disparity between those players trading for fun and those trading in a digital factory for wage-labour.

Technology also contains no specific quality or formal operation, but manifests itself as required along modes of production and class struggle. Technology works to redefine or redistribute the means of production by providing capitalist production with further methods to divide and manage labour. Marx, for example, writes about the manner in which machines require workers to constantly change or diminish their skill sets. In Grundrisse (2002), Marx explains the relationship between labourers and Capital as follows:

This economic relation - the character which capitalist and worker have as the extremes of a single relation of production - therefore develops more purely and adequately in proportion as labour loses all the characteristics of art; as its particular skill becomes something more and more abstract and irrelevant, and as 
it becomes more and more a purely abstract activity, a purely mechanical activity, hence indifferent to its particular form; a merely formal activity, or, what is the same, a merely material activity, activity pure and simple, regardless of its form. (235-236)

For example, industrial production of an item pushes work formerly done by an artisan out of the market, thereby leaving the factory as the viable mass producer of an item. Moreover, worker activity itself becomes increasingly mechanical, reflecting the increasingly malleable boundaries separating workers and the tools of industry they labour beside. Dyer-Witherford summarizes this process as follows: the major treatments of factory machinery in Capital tells a story in which capitalism, as it deepens its control of the workplace and society transforms methods of production. Marx describes this process in terms of successive degrees of 'subsumption.' In 'formal subsumption'--roughly the early stages of the industrial revolution--capital simply imposes the form of wage labour on preexisting modes of artisanal production. But in the subsequent phase, 'real subsumption,' it undertakes a wholesale reorganisation of work. Science is systematically applied to industry; technological innovation becomes perpetual; exploitation focuses on a "relative" intensification of productivity rather than an 'absolute' extension of hours. (Cybermarx 79)

This form of subsumption is possible in video games as well. As evidenced by the previous discussion of gold-farmers, the act of play has a form of wage labour imposed upon it, and thus becomes a quantifiable labour-power. Gold-farming also constitutes a form of exploitation in which those who can afford the means of production (video games 
and computers to use them) can derive benefit by hiring wage-labourers. The abstract player (gold-farming labourer) experiences an abstract payment in the form of a wage, as opposed to the product they have made (meaningful play).

As a result of subsumption, workers become quantifiable units of labour-power whose work can be measured in monetary value. Given the discussions in previous chapters, it is important to remember that virtual items can be commodities with discernible exchange-value, and producing them can therefore be an act of labour-power. Amy Wending summarizes commodification and consumption in relation to labour as follows:

Any given commodity's value can be seen either from the perspective of use or from the perspective of exchange: for enjoyment consumption or for productive consumption. Likewise, any given worker can be seen as capable of concrete labor or abstract labor-power. Labor is a qualitative relation, labor-power its quantitative counterpart. In capitalism, human labor becomes progressively interchangeable with mechanized forces, and it becomes increasingly conceptualized in these terms. Thus, labor is increasingly seen as mere laborpower, the units of force to which the motions of human work can be analytically reduced. In capitalism, machines have labor-power but do no labor in the sense of value-creating activity. (104)

Although some players might view these commodities as entertainment only (use-value), real-money transactions indicate a universal equivalent and therefore also exchangevalue. The value-creation that Wending refers to here is the production or consumption of a commodity for its own sake as opposed to its abstracted value. World of Warcraft's 
auction house, for example, might be seen as a technology which subsumes trade relationships and practices. The enchanters who formerly spammed macros to advertise their services, for example, no longer required social interaction to sell their services as a result of technological updates in the auction house.

However, abstract labour under real subsumption presumes that a worker only produces partial objects, such as on a vehicle assembly line, and is therefore alienated from any production of a use-value. The result is a working class whose value rests solely in their ability to produce abstract exchange values. Real subsumption is something that takes place broadly across social life. As McKenzie Wark's Gamer Theory (2007) contends, for example, non-game spaces are increasingly becoming gamic. Gamic interfaces and rule structures have been incorporated to effect and define social interaction in non-gamic spaces. Seriosity, for example, a consulting company that helps to gamify businesses, worked with IBM several years ago to explore "how leadership in virtual worlds may or may not be different from leadership in the physical world." Seriosity's website exclaims that "environments [online games] allow leaders, managers and anyone with good ideas to collaborate in organizations that are distributed, global, hyper-competitive and virtual" (n. pag). Seriosity took their gamification seriously, designing their virtual economy program, Attent, entirely on "solutions inspired by multiplayer games." Attent is "a monetary-based message prioritization system. Emails are visibly tagged with a currency value that signals the importance of the message." Although my paper is concerned almost exclusively with video games, these corporate examples provide context for the influential nature of video game interfaces and rule systems. These early attempts at corporate gamification are now commonplace 
methods among service jobs and tertiary industries. Bogost succinctly explains that "gamification is marketing bullshit, invented by consultants as a means to capture the wild, coveted beast that is videogames and to domesticate it for use in the grey, hopeless wasteland of big business, where bullshit already reigns anyway" ("Gamification is Bullshit”). Workers report at various jobs, for example, earning gift cards for up-selling the most cell-phone plans in a month. Instead of wage increases and benefits, workers are rewarded and paid as part of what can only nominally be called a game under Suits' definition, according to which games are composed of "unnecessary obstacles." While the interfacing of gamic logic onto real-life trade is already in practice, Bogost explains that "developers and players have critiqued gamification on the grounds that it gets games wrong, mistaking incidental properties like points and levels for primary features like interactions with behavioural complexity." Bogost asserts a similar argument that Pearce, Malaby, Sicart, and others have made about the ontological relationships which emerge between games and player interaction. Wark, on the other hand, would argue that real-life is already "gamespace," replete with similar rules and mechanics of control, and the process of gamification is simply the logical conclusion of networked society.

While terms such as playbour (Kucklich, 2005) and gamification indicate a developing tension between capitalism and games, there is yet, I think, to be a real subsumption of play, even if the rest of social life has been, according to Wark, Galloway, and others. The gold-farmer, for example, still produces a use-value among the larger MMO community by contributing to its persistent and populated nature. Although players might spend their time running shops or selling accounts and items on secondary markets like eBay, they are, at least currently, tied to the value-creation of 
play. A Marxist economic approach is essential to understanding how capital is captured, circulated, and managed in the Steam marketplace, for example. Trading is an immensely valuable component of Steam, and, as such, I spend a portion of the chapter on Steam identifying the importance of exchange to the creation of value. In addition to the Marxist literature discussed previously, I rely on Michael Heinrich's Introduction to the Three Volumes of Karl Marx's Capital to explain the production of value central in Marxist economics. Heinrich's reading of Capital provides an excellent description of the construction of value through exchange. I will return to this conversation of labour and play in the section on the Steam Market.

\subsubsection{Mass Media, Networks, and Culture}

From a cultural perspective, Marxist critique of mass media, including video games, is primarily influenced by the Frankfurt School. In their seminal text, Dialectic of Enlightenment (1944), Adorno and Horkheimer rail against mass culture for propping up and reinforcing capitalist-driven political and cultural hegemonies. Writing about the connection between business and entertainment, Adorno and Horkheimer explain that the original affinity between [the two] reveals itself in the meaning of entertainment itself: as society's apologia. To be entertained means to be in agreement. Entertainment makes itself possibly only by insulating itself from the totality of the social process, making itself stupid and perversely renouncing it from the inescapable claim of any work, even the most trivial: in its restrictedness to reflect the whole. Amusement always means putting things out of mind, 
forgetting suffering, even when it is on display ... It is indeed escape, but not, as it claims, escape from bad reality but from the last thought of resisting that reality.

It might be an exaggeration to criticize an interface function of contemporary MMOs for contributing to the type of exploitation that global capitalism perpetuates, but I contend it is necessary to examine, and hopefully dismantle, the seemingly innocuous processes which encourage players to exist comfortably within this exploitation. Adorno and Horkheimer insist that the "rigid institutionalization" of mass culture works towards an "undreamed of psychological control. The repetitiveness, the selfsameness, and the ubiquity of modern mass culture tend to make for automatized reactions and to weaken the forces of individual resistance" (476). The design and game theorists I discussed under the Interface section of this project support, albeit with occasionally different interpretations, the critique levied by Adorno and Horkheimer; games, like any media system, are systems that reward players for behaviour patterns that acknowledge (and perhaps tacitly support) algorithmic processes which are laden with ideology.

The commodified game culture that Malaby documents in a game like Second Life, for example, reflects the horrifying reality of contemporary mass culture for Adorno and Horkheimer: "the compulsive imitation by consumers of cultural commodities, which, at the same time, they recognize as false" (136). Additionally, the constant pressure in game play to acquire and trade valuable items recalls Adorno and Horkheimer's assertion that "anyone who does not conform is condemned to an economic impotence which is prolonged in the intellectual powerlessness of the eccentric 
loner. Disconnected from the mainstream, he is easily convicted of inadequacy" $(106){ }^{20}$

The social life of the game exemplifies the "pseudo individuality" that Adorno and

Horkheimer see as integral to contemporary capitalism. The myth of rugged

individualism is the escapist fantasy in most games, including MMOs, and the player is

meant to experience the world of a hero. Yet, all players experience the same gameplay

in accordance with the mechanics set out before them. Individualism is asserted only

insofar as its characteristics are predetermined and prescribed. The house that Malaby

desires, and the decorations he purchases, are all scripted by programmers. The promise

of individual self-realization in mass culture, according to Adorno and Horkheimer, is

illusory: "individuals are tolerated only as far as their wholehearted identity with the

universal is beyond question" (124).

Contemporary MMO game designers rarely provide varied and interesting social

spaces or mechanics, and instead produce superficial content designed to encourage the

same repetitive and laborious play its user base pays for via monthly subscription. ${ }^{21}$ Ian

Williams writes that "gamer identity is tainted, root and branch, by its embrace of

consumption as a way of life. .. . Practical ceilings on consumption and

compartmentalized identity seal people off from their fellows even as they find

20 Although the economic destitution that Adorno and Horkheimer are writing about is based on actual poverty, games can become sites of class stratification. Jennifer Martin, writing about Second Life, explains that "virtual world consumption has the potential to add another dimension to the reproduction of social inequality in virtual worlds. It is possible, if not likely, that within a virtual world that offers consumption, and indeed encourages it, the goods with which they are associated will mark virtual bodies and lives. The fact that consumption in virtual social worlds is not only linked to offline economies but readable in these terms intensifies the issue. Not only is the virtual body marked by consumption, but the goods that are consumed have the potential to be marked by indicators of offline status, such as wealth, that could be problematic for egalitarian online interaction" (41).

21 Level caps are an excellent example of this type of game design, and something that Blizzard has excelled at in managing World of Warcraft over the last decade. Initially, characters stopped levelling up at level sixty. Level caps have been subsequently raised over the course of six expansions. 
commonality within their consumption cohort" ("On Geek Culture"). Contemporary MMO game design reflects the diminishing spontaneity that Horkheimer and Adorno identify as characteristic of mass culture under capitalism. Recently, however, game designers and players have responded to this feeling of homogeneity, as I will discuss in my chapter on indie games. Emergent cultures require heterogeneous game spaces to exist. As contemporary MMOs produce the same prevailing interface designs, such as trade interfaces, the social and cultural potential of MMOs becomes increasingly stagnant.

As a result of an increasingly commodified culture of play, social interactions within online games have become emblematic of capitalist and corporate culture, evidenced by the militaristic and hierarchical structures of contemporary in-game guild life and the fusion of online and offline economies. ${ }^{22}$ I do not mean to argue that the infusion of capitalist thought into games begins with trade interfaces (arguably it has existed from the inception of video games), but that trade affordances demonstrate the ways in which capitalist ideologies can entrench themselves in seemingly innocuous procedures. The ubiquity of trade interfaces coincides with and enables the growing tensions surrounding in-game purchases for real-world currency by assuring players that their items will be safely transitioned. Ultimately, interfaces influence the political possibilities of players whether players are aware of it or not. Trade interfaces codify notions of private property by ensuring that items acquired are always viewed as extensions of players' labour time. In a world of interfaced exchange, games appear more and more like stock markets, reflecting market value and currency adjustments for

22 In an interview on The Daedalus Project, Nick Yee interviews a player who compares guild structure to military structure ("The High-End Game"). The player, whose character name is Talon, describes the importance of discipline and hierarchy in successful guilds. 
"useful" in-game items. A paragon of cynicism, the trade affordances trust no human. Instead, the exchange of items of "equal worth," if such a thing is even possible, is monitored and enforced.

For recent game studies critics, video games are paradigmatic of capitalism because they are both representative and literal embodiments of the modes of oppression and control in contemporary social life. This dissertation follows these theorists in attempting to articulate and explain the methods of control employed by capitalist ideology through video games. The data presented in this research also indicates that players feel increasingly alienated from their own communities of play. The feeling of alienation among players is, in no small measure, the result of the standardized processes by which materials are traded within the community.

Other Marxists expanded upon and updated where necessary the ideas put forth by Adorno and Horkheimer. Generally speaking, these Marxist scholars match their critique with advancements in technology. Herbert Marcuse's One Dimensional Man (1964), for example, posits the argument that contemporary mass media, advertising, bureaucracy, democracy, and other tools of capitalism have flattened social life into a single dimension. Marcuse explains that capitalism creates false needs in order to alleviate the anxiety and ennui that citizens feel under this regime of social control. Consumerism, for Marcuse, is a method of social repression intended to prevent any revolutionary action which might threaten the accumulation of capital by a select few. The increasing rate of consumption among video game players indicates, perhaps, the homogenization of virtual worlds as well, thereby resulting in a decline in their potential to enact meaningful political or cultural decisions. 
More recently, Nick Dyer-Witherford and Greig de Peuter suggest that games "recapitulate patterns of online shopping, social networking, and digital labor crucial to global capitalism" (Games of Empire xiv). These scholars often situate their critique of video games in relation to social power by closely considering their unique media properties, but are careful, as Dyer-Witherford and de Peuter explain, "to never assume they simply transcend 'old-media' problems of ideology and political control" (xxvii). Additionally, these critics define their critique according to the historical material conditions under which they find themselves. Vincent Mosco's Digital Sublime (2004) is a response to what he sees as the techno-positivist impulse that marked game and digital scholarship throughout the 1990s and early 2000s. Mosco demonstrates the manner in which the means of communication have become increasingly consolidated despite the belief in the democratizing power of computer networks. Additionally, Mosco systematically dismisses the various argument made by neoliberals and futurists, such as Francis Fukuyama and Alvin Toffler, regarding the end of history and the irrelevance of Marxism in the information society. Of note, Lisa Nakamura's Cybertypes (2002) performs a similar dismissal of early so-called cyber-utopianists, such as Janet Murray, who professed the liberating powers of the web with regards to gender and race. With the rise of cosmetic items and in-game purchases, it turns out, players can look as impoverished in game as they are in real life.

Dyer-Witherford explains that proponents of the "information society" believed technology would spread to countries in a trickle-down process reminiscent of Smith's invisible hand, thereby providing pre-industrial nations with the ability to leap-frog industrialism and join the "global village" of which Marshall McLuhan dreamed 
(Cybermarx 43).Instead, capitalism expanded its ability to abstract value from social life by employing channels of communication. Tiziana Terranova (2004) explains the makeup of contemporary capitalism as follows:

[T]he communication topology of Empire is complex as it is woven together by aeroplanes, freight ships, television, cinema, computers and telephony, but what all these different systems seem to have in common is their convergence on the figure not simply of the network, but a kind of hypernetwork, a meshwork potentially connected every point to every other point. As such, the network is becoming less and less a description of a specific system, and more a catchword to describe the formation of a single and yet multidimensional information milieu - linked by the dynamics of information propagation and segmented by diverse modes and channels of circulation. (Network Culture 40-41)

Terranova here refers specifically to Michael Hardt and Antonio Negri (2000), who define Empire as the "center that supports the globalization of productive networks and casts its widely inclusive net to try to envelop all power relations within its world order" (Empire 20). Hardt and Negri argue that Empire can "no longer be adequately understood in terms of imperialism as it was practiced by the modern powers, based primarily on the sovereignty of the nation-state extended over foreign territory," but instead acts as a global networked power whose constituents include nation states, financial institutions, and multinational corporations (Multitude xii). For Hardt and Negri, Empire is a multi-modal expression of biopolitical control.

Similarly, Dyer-Witherford and de Peuter's Games of Empire (2009) demonstrates "how video games, hacked into existence forty years ago by a Pentagon- 
mobilized technical workforce as part of vibrant freeware culture, were captured by entrepreneurs, commodified, and transformed into a colossal corporate complex. Today this process culminates in a situation where virtual games are being sent 'back to work,' where they are used as a means of training new generations of immaterial labor across all sectors of capital" (xxx). Games of Empire draws on Hardt and Negri's work on cognitive capitalism and affective labour, and particularly on the "distinctly cognitive dimension" of property through which contemporary capital thrives (37). DyerWitherford and De Peuter alternatively refer to immaterial labour as "cognitive capitalism," which "refers to a system of production in which knowledge plays an integral role.... Cognitive capitalism therefore emphasizes the dependence of corporate enterprises on the thinking - the cognition - of its workers, and the distinctly cognitive dimensions" of property (36). In Games of Empire, they contend that "video games are a paradigmatic media of Empire" which represent the "emergence of a new planetary regime in which economic, administrative, military, and communicative components combine to create a system of power 'with no outside"” (xiv, xix). Hardt and Negri's description of Empire, like Dyer-Witherford and De Peuter's, begins with the idea that immaterial labour is the driving force behind material production and exploitation. For example, video games drive sales of increasingly complex software and hardware, which in turn requires and encourages the material exploitation of something like cobalt mines.

Similarly, Wark's A Hacker Manifesto (2004) discusses the manner in which property rights have enveloped intellectual and creative efforts, thereby creating what he refers to as a "vectoralist class." According to Wark, the vectoralist class manages the channels through which information and intellectual property travel, and in so doing is 
able to manufacture scarcity and profit from these restrictions. Trade interfaces are one of many examples of networked, vectoralist processes Wark describes. Although trading takes place in games through player interaction, all trading is framed by the interface that the game provides. Any movement of items can only happen through the vectors provided by the trade interface. The exchange of items becomes a monitored and regulated process, guided by an interface as opposed to a community. Trade interfaces, then, are an interface composed of vectoralist logic. Although scarcity is programmed into games, insofar as there is a limit to how many items or rare objects might be acquired, trade interfaces work to codify this scarcity by encouraging players to interact with these digital items as if they are inherently scarce, part of a larger commodified culture, and removed from the communal narrative online games are capable of producing. Trade interfaces can be seen as an institutional operation through which the transformation of digital information in the form of items, currency, and other abstractions are managed. Trade interfaces are a tool that capital employs to accumulate new value through new markets. I will, in their respective chapters, discuss how each object of study in this dissertation manages this informational exchange. While trade interfaces demarcate the boundaries of play, there are games, communities, and developers who have resisted the growing abstraction of play into labour-power.

Opposite Hardt and Negri's Empire, however, exists the Multitude, which reaffirms the potential for people to resist capital and re-appropriate technologies and social conditions for their own purpose. Similarly, Dyer-Witherford, writing from an Autonomist perspective, view workers (he would also add players) as the active subjects of production, thereby disregarding the all-encompassing qualities of real subsumption. 
Instead, Autonomist Marxists view labouring subjects as the enabling entities of technology and capital, but not the other way around: “capital, a relation of general commodification predicated on the wage relation, needs labour. But labour does not need capital" (Cybermarx 137). While Marx writes about the oppressive qualities of technology, he is careful to assert that this is a condition of capitalism and not an inherent quality of the machinery. Amy Wending elaborates that "beneath the surface of the antagonistic relationship between human and machine is a deep class kinship that mirrors the class kinship of proletarians amongst themselves, and that suggests a different mode of production would enable better relationships between humans and machines" (100). As technology concerns modes of production, a change in this mode would precipitate a revolution in the relationship of human and machine.

Marx insisted that individuals possess agency and freedom but operate under institutional and ideological constraints that shape their understanding of the world (Little 6). Despite their differences, the research and literature discussed so far tends toward a prevailing opinion that humans operate with consciousness and agency, but find themselves under duress by a host of social, cultural, and economic rule structures. A notable exception being that most neoclassical and classical economics tend to construct human beings as reasonable and free actors regardless of the system in which they find themselves. Therefore, I think it is necessary moving forward to recognize that trade interfaces - from trade windows to auction houses to Steam markets, including human interfaces in the form of MPs - hold a significant account of influence over play patterns and player decisions. Moreover, their influence can be thought of as a social or cultural indicator of the locations of power, alienation, and exploitation under capitalism. From 
the auction house, for example, one can view the development of gold-farming, and from there it is possible to spot inequality between nations or between individuals under capitalism. As Dyer-Witherford beautifully explains, "in a classic dialectical trope, historical materialism has been dematerialised" (Cybermarx 47). 


\section{Chapter 2:}

\section{Interpersonal Exchange in The Realm and World of Warcraft}

Before trade affordances there were players. Instead of beginning with classical economics, which proclaims trade to be a by-product of consumption and production, this chapter will begin with the Autonomist assumption that trade is a necessarily productive task that begins with human interaction. Inverting these classical assumptions, this section will address the interpersonal and social aspects of trade that produce value. Trade is not necessarily secondary to production or consumption, and can be a form of labour in and of itself. Dyer-Witherford explains that Marx "focused on the power of capital to direct production through the accumulation of machines. In Autonomist theory, however, this emphasis is reversed: the analysis of class composition is aimed at assessing the capacity of living labour to wrest control away from capital" (Cybermarx 133). As a result, I use this section of my discussion to argue that human intermediaries or MPs in The Realm constitute a class composition under duress from the logic of capital. Moreover, I propose that the shift from human intermediaries to trade interfaces represents a formal subsumption of work and abstraction of play in labour.

Hardt, Negri, Bologna, Dyer-Witherford, and other Autonomists categorize the twentieth century into three separate epochs of class conflict: the time of the professional worker, the mass worker, and the socialised worker. In the early twentieth century, professional workers had largely moved to factories, but retained knowledge and skills prior to subsumption. According to Dyer-Witherford, following the Bolshevik revolution, capital attempted to disempower and disenfranchise these workers by further 
stratifying their production, resulting in the Fordist model of production (145).

Additionally, the threat of revolution caused the government in the US and other Western countries to intervene in social and economic issues (McCarthyism, for example). The resulting mass workers, however, formed class solidarity to fight for living wages and bargain with capital for union pay and benefits. Additionally, Autonomists note the production of the social factory during this period. Dyer-Witherford summarizes this period as follows:

The gendered division of labour and the pairing the male mass worker--whose life is to be slowly obliterated on the assembly line--with the female housewife, whose lot is to tend the wounds, take the abuse, do the shopping and raise the next generation of labour power in the isolation of the home-- becomes a conscious concern of capital's social managers. The labour of the female housewife, whose 'consumerist' schedule is organised largely through new organs of mass communication, such as radio and television, starts to become as much the object of a corporate planning as the productivity of her male partner on the shopfloor-for it is through her activity that the pay increases won by the mass worker are translated into the consumption necessary for a virtuous cycle of continual capitalist growth and stability. (146-147)

The result of this management of social life and factory work, however, led to protests by mass workers, as well as new social movements along race and gender lines. In response, capitalism demolished much of the Fordist organization of mass labour. Assembly lines and factory jobs began to disappear in favour of corporate conglomerates managing information and data. According to Dyer-Witherford, these changes began in the 1970s, 
developed throughout the Thatcher and Reagan era, and solidified in the 1990s (50). Additionally, businesses and governments developed an interest in communication technologies previously under the purview of the military. According to Dyer-

Witherford, by allowing "centrally controlled, comprehensive factory automation and the splitting-up of the production cycle, management can now reduce and disperse workers once concentrated together so they are scattered territorially, socially and culturally, in different conditions of work and often invisible from one another" (155).

The result of this process is the birth of the socialised worker, who exists in what has been variously described as cognitive capitalism (Virno, Berari), information capitalism, Empire (Hardt and Negri), vector capitalism (Wark), knowledge economy, and social capitalism. Although I think capitalism is also acceptable without the descriptor, as it encompasses all of these sources of labour and more, explanatory phrases such as vector or information can help to delineate between new forms of knowledge economy and older forms of, say, mass production. The general qualities of the knowledge economy are a diffuse and decentralized workforce whose skills and work hours are flexible, malleable, and technology-driven. Wage labour is decreased in this imagination of capitalism, but temporary or casual work increases. There are numerous cases of informal work, unpaid internships, and irregular schedules. There is an increase in tertiary or service industries reliant on affective labour. Additionally, value is abstracted from social life in accordance with the new technical means of production. Data of all sorts develops an appreciable exchange-value and begins to determine certain modes of production. For example, Jodi Dean explains in her study of blogs that simple participation in social media alone results in the creation of value because of data, 
advertisements, and other informatics. "Productive labour," according to Negri, "is now that which produces society" (qtd. in Dyer-Witherford 150).

Autonomists and Marxists are still rightly concerned with the material and physical conditions of labour. Moreover, Autonomists assert that their history of twentieth-century class conflicts does not entail that "workers of one era vanish at the commencement of the next: professional workers persist into the era of the mass worker, and mass workers into the epoch of the socialised worker: the point is that the innovative and strategic centre of struggle shifts" (Cybermarx 258). Moreover, these cycles of struggle are meant to describe broad shifts within global capitalism and can and should be modified to address local concerns where necessary. Additionally, it is important to note that, although play and design often operate as the ideological expression of capitalism within games, actual physical labour is exploited in order to acquire the material products necessary for games. While the majority of this dissertation is spent addressing the manner in which games enact ideological reproduction, it is important to remember that workers suffer in sweatshops in the global south assembling computer parts in factories, and miners are forced to produce copper for gaming machines at gunpoint. ${ }^{23}$ The point here is that the arrival of information capital did not eliminate previous forms of class struggle.

\subsection{The Realm and the Socialised Exchange}

Christian Fuchs's Digital Labour and Karl Marx (2014) begins by listing numerous instances of ruthless exploitation of workers by massive corporations around the world. Examples include enslaved miners in the Democratic Republic of Congo, Chinese engineers who built the first iPads, and information technology workers in Silicon Valley who were exposed to toxic substances. 
The Realm, then, comes into existence as capital expands into new technological markets: online video games. As mentioned previously, The Realm marked a transition between MUDs to MMOs, and demonstrated the burgeoning economic production associated with these games. In light of the recent resurgence of interest in the game, a community forum post highlights the experience of some of the game's early adopters: Sierra originally placed the game on AOL. NWN was still played as a MUD on AOL, then along came the testing of The Realm. I was a ToS moderator for AOL at that time. Most of the Mods got a message from mgmt to go and test this new and exciting adventure game, called The Realm

The crowds there, at that time, were miniscule. Very little content and monsters were single digits. Word started getting out, more people wanted to play, which was good for Sierra, so they left AOL and took it live out into the world of the new internet. 4800 baud rate and we thought we were cooking!

THEN!!! Someone invented ICQ! Those of us, in game, now actually had a way to chat without having to use AOL. Now we could tell people where we were in the que for logging in. Other companies started making dial-up available. I remember switching from AOL to MindSpring and, omg!, I got 9600 baud! Life was good on Realm. (Community.TheRealm "LadyCrystal" n. pag.)

This particular forum post is illuminating for historical, ethnographic and theoretical reasons. ICQ is an early instant messaging program, baud refers to her dial-up speed, and NWM refers to Neverwinter Nights. Notably, ICQ and other messaging programs 
assisted gamers whose dial-up usage prevented them from using the phone, or who could not afford the long-distance charges. Furthermore, it indicates the concomitant development of MMOs and Internet technologies, as well as the experimental nature of these early games.

More importantly, this forum post indicates the existence of a flexible and diverse labour that constitutes contemporary knowledge economies. Terranova notes, for example, that AOL mods were rarely if ever paid but essential to the development and growth of the company (Network Culture 92). Free labour, Terranova explains, is a fundamental aspect of network culture. Not only was this particular user a mod, but she thought of testing not so much as labour, but as a participatory privilege. There is, of course, voluntary engagement on behalf of users and players who are simply happy to play the game. However, this is an irrelevant detail from the perspective of capital. Certainly these players or users got value out of their time, be it free use or socialization, but value is not a measure of finances alone. Not even capital cares about wealth, and it is a common mistake to think so. What capital is interested in more than accumulating wealth is accumulating manageable labour sources. There were no shortage of early adopters, as Terranova documents, who were willing to mod, play test, or perform other free labour.

This post also demonstrates a user who Autonomists might describe as a socialised worker. One of the key features of socialised workers is their ability to adapt. In fact, the name comes from Negri's attempt to combine The Road to Wigen Pier-style working class ethos with the new social movements of the civil rights era. DyerWitherford explains that "to be socialised is to be made productive, and to become a 
subject is to be made subject to value--not only as an employee but as a parent, shopper and student, as a flexibilised home worker, as an audience in communicative networks, indeed even as a transmitter of genetic information" (158). Autonomists view socialised workers as potentially radical identities that span multiple boundaries of experience and belief, and therefore constitute a powerful response to capital's diffuse, knowledge-based mode of production. Dyer-Witherford describes the manner in which socialised workers can navigate a "system of social machines," and thereby "this techno-habitat becomes so pervasively socialised that it can no longer be exclusively dictated by capital" (163). Unlike neo-Luddites, Autonomists desire the re-appropriation and reintegration of technology.

The Realm's MPs, in some ways, reflect this desire for reappropriation and reintegration. However, this reflection happened unintentionally, not through any sort of radical action performed intentionally by MPs. Furthermore, I do not mean to suggest that The Realm's MPs or players operate outside of capital. Instead, they represent a meaningful alternative to the standardized methods of trade that imagine human exchange as nothing more than quantifiable data. Moreover, because MMOs were only emerging, the influence of capital had not, presumably, affected the whole of social life and exchange in the game. It is interesting to note that, given the existence of auction houses and markets, the design team was clearly aware that players would engage in trade with one another. Despite their acknowledgement that players would desire to trade with one another, the designers provided no other technical affordance to exchange an item from person to person other than a "give" command.

Writing about gift economies, Marcel Mauss explains that exchange within these 
systems is not solely a matter of producing markets and wealth for individuals, but also one of establishing social contracts and obligations. Mauss insists that groups participating in gift economies are not exclusively exchanging "goods and wealth, real and personal property, and things of economic value. They exchange rather courtesies, entertainments, ritual, military assistance ... and fairs in which the market is but one element in the circulation of wealth but one part of a wide enduring contract" (3). Both Mauss (1990) and George Bataille (1988) describe trade and production as "the effervescence of life," and in so doing insist upon the emergent social relations that form within the circulation of commodities. Even Marx referred to labour as "the living, formgiving flame" (qtd. in Dyer-Witherford 132). The persons involved in these contracts, Mauss explains, often involve intermediaries (be they families, clans, or close-knit social groups) who assist in exchanges. Relevant here is Mauss's interest in the possibility for trade to produce value through interpersonal relationships. The Realm was by no means a gift economy, nor an ideal system for transgressing capitalism as a game, but was able to produce trade relationships outside of mere exchange-value. Interestingly, the player guide I mention in my earlier description of The Realm describes trading as a "dangerous, but potentially rewarding experience" ("Trading Guides!" n. pag.). Frank Ross, the Codemasters developer who was running the game in 2003, explained that he had spent a fair amount of time researching the reasons players were still playing The Realm years later, despite more advanced MMOs on the market ("Interview" n. pag.). Ross concluded that the social interactions the game required, from trading to navigation, resulted in meaningful game play experiences for some players. The lack of interfaces for both trading and navigation required players to interact, and these interactions in turn 
produced valuable socialization for some players.

The relationship between players, social value, and rule systems hearkens back to Pearce's explanation of how the manner in which mechanics (or a lack thereof) can produce unexpected and noteworthy play patterns. As a result of these human intermediaries, social and moral role-playing developed around trade interactions. Players began to view themselves as part of a community with a history and a story, with some players acting the villain and others the heroes. Players who engaged in roleplaying "good" characters versus "evil" characters, for example, may have engaged in a career as an honest middle-person, while others may have plotted various scams. Regardless of a player's choice, an emergent culture, and what Pearce terms productive play, arose out of the social interactions encouraged by the absence of trade interfaces. For Pearce, productive play is a form of play that "merges with creative production" ("Narrative Environments" 203). Pearce explains that "players are not simply spectators, but rather take the roles of elves and orcs fully engaged with the narrative and conflicts of the game. Unlike at a costume party or on Halloween, however, these identities are 'persistent,' meaning the player maintains the same role over time" (203). In fact, some players spent their time on the fringes of the game, choosing to engage solely in modes of productive play rather than following the prescribed grind through the game. Therefore, MPs produced use-value that resisted abstraction into exchange-value.

All of these communal features assert the importance for many players to determine their own values and standards of play. By trading through human intermediaries, players forge bonds and establish community values through the act of trading. Players are able to determine, within the boundaries of the game world, the 
ethical construction of their play. Even deviant acts like scamming in The Realm can function as community-building moments. Nancy J. Herman's Deviance (1995), which summarizes Emile Durkheim's structural-functionalist approach, explains that deviance can be essential in demonstrating community standards and reaffirming a particular society's solidarity (33). Moreover, Herman explains that Durkheim imagines deviance as a precursor to social change (34). However, this social change does not always indicate a positive or desired social change, and can result in what Durkheim referred to as anomie, which Herman defines as "a state of normlessness or social deregulation" (33). For Durkheim, this state of affairs is brought about by the division of labour, which produces distinct and competing interest groups who have divergent perceptions on right and wrong. This phenomenon is visible, for example, in the disruption of achievement hierarchies that Lehdonvirta documents, as real-money transactions begin to compete with players' conception of fair play.

Yee describes a similar contention between regular players and gold-farmers on his Daedalus Project as well. It is worth noting that the division of labour in these two examples is both technical and economic. From a technical perspective, the trade affordances of these games allowed players to exchange items securely and efficiently such that players' economic divisions came to bear on the social life of the game. The Realm, however, was incapable (for technical reasons at the very least) of providing a similar economic model, and as a result can be imagined as a space in which social regulation takes precedence over capital. The ability to "give," whether resulting in a scam or not, prevented economic stratification via real-money transactions, while encouraging socialization through technical inadequacies. 
Lehdonvirta explains that "social relations are central for deriving value from [digital] goods. The bits that constitute virtual goods have little experiential value without meanings attached to them within a social world. The prerequisites of social control are therefore in principle present when virtual goods are consumed, but the question is what the nature of the social world is like: caring or indifferent" (67). Trade interfaces remove possible social relations from trade, however, and produce indifferent consumers. Trade interfaces accomplish this in part by undermining the potential emergent diegetic and interpersonal relationships possible from trade between one player and another. Instead of two imagined player-characters engaging in trade, trade windows facilitate trade between two individual players, separating the diegetic world of the game from the player. In so doing, players are left without the social relationships forged through trading with one another. The social trade practices in The Realm demonstrate alternative modes of play which emerged from the absence of what is now standardized interface design.

Applying the earlier theoretical discussion on subsumption and the diminishing of labourers' skillsets, there is also a parallel to be made with the manner in which machinic operations have replaced some players' roles in the community. In fact, the advent of trade interfaces makes this style of play completely obsolete, thereby lowering the possible methods of social interaction available to players. A job once considered an act of play and socialization has been wrested from away from humans and made the task of a machine. A similar history takes place in World of Warcraft with regards to auction houses, as player exchange became increasingly automated by updates to the auction house affordances. 


\subsection{The Material, Social, and Cultural Implications of the Auction House.}

As previously mentioned, auction houses in World of Warcraft are one of the primary methods for farming in-game items and gold. In this section, I synthesize Sicart's video game ethics, Georg Simmel's writings on monetization, and Alexander Galloway's semiotic squares in order to assess the ethical and socioeconomic function of auction houses. Additionally, comparing the MPs of The Realm to World of Warcraft's auction houses demonstrates the automation of labour and abstraction of intersubjectivities that enable the generative economic forces surrounding World of Warcraft.

In Interface Effect (2012), Alexander Galloway attempts to understand the social and cultural histories embedded in the metaphors of interface. In other words, Galloway examines how contemporary cultural authority and forms of oppression can be located in technological design. For example, personal computers and desktops have become the tools through which much of our contemporary social, political, cultural, and personal lives are mediated, and so have become spaces of extraneous physical and affective labour for human beings. Raw value is extracted by data-mining users of social media, for example, by advertising profiteers. Regardless of any intended use of personal computers, people are performing more and more labour each time they log on to play a game, browse news websites, or use search engines. Interfaces, then, are not just for communication between humans, but also for communication between humans and banks, corporations, or government institutions. The desktop metaphor is no longer (and 
perhaps never was) merely an improvement of work space, but rather represents a shift in the possibility for disseminating, promoting, controlling, and regulating cultural and societal systems.

For this reason, Galloway claims that interfaces are "not a thing," but instead "always an effect" (28). As the various researchers in the section on interface design indicate, interfaces such as auction houses can work to prescribe particular social behaviours and interactions. Trade interfaces frame play in a manner that undermines role-playing, narrative, and agency while promoting efficient item micromanagement and acquisition. Narratives about trade and trade participants are limited by the auction house's machinic interruption of player-characters' social interactions. As a result, interfaces such as trade windows raise the question of how labour is performed and for whom it is performed in the act of game play. Galloway contends that "each part of the interface has its role to play .... The existence of the internal interface within the medium is important because it indicates the implicit presence of the outside within the inside. And, again to be unambiguous, 'outside' means something quite specific: the social" (42). As an interface, auction houses work inconspicuously to bring the social into the game by reproducing capitalist ideologies about property and ownership. Recall that one of the main goals of this dissertation is to examine how we might begin to criticize the connections between specific interface operations and the socioeconomic problems of capitalism.

In Gaming: Essays on Algorithmic Culture (2006), Galloway employs a semiotic square to define gamic moments. This construction is made up of diegetic, non-diegetic, operator (player), and non-operator functions (17). An operator moment are those in 
which players have decisions to input or interact, and non-operator moments are the noninteractive rule structures or mechanics. In this semiotic square, a diegetic operator element, for example, is a moment in which a player's action contributes to the overall development of the game's narrative or world. A non-operator diegetic function is something like a cut-scene, which advances the world's narrative but is not interacted with by the player. A non-diegetic operator element, for example, might be a menu or item screen which the player selects from. The auction house, for example, is a (mostly) non-diegetic operator function. Players interact with the menu but it does not necessary impact the narrative of the world. MPs in The Realm, however, obfuscate the distinction between the non-diegetic and diegetic axis by enabling trade, and in so doing create a narrative role-playing world.

Galloway's later work in Interface Effect expands upon this method by including a second quadrant of coherent, incoherent, politics, and aesthetics. Coherent and incoherent, for Galloway, are "nonnormative terms; they must be understood more as 'aligned or unaligned' or 'fixed or not fixed' rather than 'good or bad' or 'desirable or undesirable"' (47). Galloway constructs politics in a diverse fashion by describing it as the organization of social life through governance, family life, work, and various other civil groupings. Galloway ascribes various characteristics to each possible combination of the quadrant, as well. The combination of coherent political and coherent aesthetic qualities results, for example, in ideology or propaganda. An incoherent aesthetic combined with coherent politics, on the other hand, creates an ethical quality. This combination is where Galloway imagines video games and their interfaces to reside. He describes poetics and art-for-art's sake as a formulation of coherent aesthetics and 
incoherent politics. Finally, an incoherent aesthetic and politic, he claims, resides in the realm of "truth." He contends that this form is elusive and has "never achieved any sort of bona fide existence in modern culture," and thus largely forgoes its discussion.

Interestingly, he notes that Georges Bataille's work, quoted earlier in the chapter on The Realm describing trade as the "effervescence of life," lies somewhere in this configuration.

Galloway combines these semiotic squares to perform close-reading of a screenshot from World of Warcraft that demonstrates the aesthetic and political powers of interface operations. He argues that World of Warcraft's game play provides an example of an incoherent aesthetic, as evidenced by the game's technical "apparatus (statistical data, machinic functions, respawn loops, object interfaces, multithreading, and so on), while all the time promoting a particularly coherent politics (phonological organization, networked integration, alienation from the traditional social order, new informatic labor practices, computer mediated group interaction, neoliberal markets, game theory, and so forth)" (49). For Galloway, then, World of Warcraft is an ethical construction, and its social elements are driven by its non-diegetic components. His assessment comments on specific interfaces within the game, such as HUDs, chat displays, trade windows, or character creation menus.

In Galloway's argument, a game's programming mostly determines the ethical construction it might have. He explains that "World of Warcraft is an 'ethical' game simply by virtue of the way in which it opens up the aesthetic on the one hand while closing down politics on the other" (49). His reading of World of Warcraft appears cynical regarding player agency, as it denies any aleatoric or emergent possibilities for 
online gaming communities to create their own modes of play that extend beyond the game's programming. In other words, the problem with Galloway's square is an assumption that game play is a closed process between these sectors. In fact, the political coherence that any MMO demonstrates is not completely monolithic, as specific lapses in interface design or programming can produce social functions that take their place, such as MPs in The Realm. Occasionally, there are moments in game play that offer spaces for communities to produce their own politics that may or may not reinforce dominant assumptions. Sicart's commentary on the honour system, for example, demonstrates the ability for players to alter the politics of any particular interface. Moreover, Chapter 3's discussion of the Steam Market and esports will demonstrate the capability of players to produce meaningful social interactions above or through aesthetically incoherent and politically coherent trade interfaces.

The difference between Sicart's optimism and Galloway's cynicism regarding ethics can be determined by their opposing ontological approach to games. Galloway contends that the "machine is an ethic because it is premised on the notion that objects are subject to definition and manipulation according to a set of principles for action. The matter at hand is not that of coming to know a world, but rather that of how specific, abstract definitions are executed to form a world" (23). Galloway, unlike Sicart, positions the ideational influence of code, mechanics, and rule systems as a primary nature of games. For Galloway, rules are enacted through play, but for Sicart, play interacts with rules. Sicart, as previously mentioned, acknowledges the severe constraints through which players operate, but insists that video games, and thus their rules and mechanics, come into being through player interaction. Galloway, on the other hand, 
seems to position rule structures as an ontological foundation of video games, and their political rules are manifested through incoherent play patterns. Even for Sicart, however, auction houses might be defined as an unethical construction with regards to trade because they severely limit players' ability to make substantial decisions about their community behaviours and customs.

Nevertheless, the auction house can be described as unethical in its coherent political construction and intention. Galloway correctly identifies that the social elements of World of Warcraft are largely determined by the game's non-diegetic technical components. For recent game studies critics like Dyer-Witherford, de Peuter, and Galloway, video games are paradigmatic of capitalism because they are both representative and literal embodiments of the modes of oppression and control in contemporary social life. I follow Galloway in attempting to articulate and explain the methods of control employed by capitalist ideology through the auction house. However, I perform my close-reading of the auction house with the caveat that not all motivations or handlings of auction house trading are known or understandable, and that players can potentially produce a variety of player-based ethics in using them. More than the other trade systems I describe, however, auction houses in World of Warcraft work toward a particular political goal of organizing social life with regards to trading. Moreover, because auction houses are so keenly associated with gold-farming and the accumulation of wealth, both in- and out-of-game, they mark a particularly strong ethical moment, in Galloway's terms, regarding the relationship between video games and capitalist ideology.

The effects of auction houses, then, are to prescribe particular social behaviour 
and interactions outside of the game. My critique of auction houses is similar to the critiques of mass culture made by the Frankfurt School. Moreover, they work to enable the uneven development and accumulation of capital through virtual items. As an interface, they work inconspicuously to limit the social elements of gameplay by reproducing capitalist ideologies about property and ownership. As Paolo Pedercini explains, "playing games per se may appear as the ultimate non-instrumental activity, the perfect antithesis to economic production and reproduction. But the act of playing, especially a computer-assisted, cybernetically-biased variety, can cultivate the capitalist mindset and value system — regardless of what the specific games are intended to portray or narrate" ("Videogames and the Spirit of Capitalism" n. pag.). In this vein, trading in World of Warcraft is framed by an interface the game provides, and the exchange of items becomes a monitored and regulated process that is guided by mechanics as opposed to social interaction. Moreover, while scarcity is programmed into the game, insofar as there is a limit to how many items or rare objects might be acquired, trade interfaces, like auction houses, work to codify this scarcity by encouraging players to interact with these digital items as if they are inherently scarce, part of a larger commodified culture. As a result, these items acquire fixed exchange values, and the auction house ensure players can efficiently trade items of set prices in an organized and impersonal manner. Recall, for example, the various wiki guides that explain the auction house's utility for players to check broad market prices for items.

Georg Simmel's description of the shift toward rationalization and monetization in modern life provides a framework for understanding the ways in which auction houses structure social game play. Mass currency and item exchanges are not possible without 
these auction houses, as players would have to drop the gold at a specific location within the game world or have a third party deliver it after picking it up in avatar. Trade interfaces in World of Warcraft, including the auction house, trade windows, and intercharacter mailing systems, allow for mass transactions, making it much easier to supply aggregated currency and items. This rationalized and quantified trade practice is a central contributing factor to the toxicity and regressive social relationships reported by MMO ethnographers like Yee, for example. In “The Metropolis and Mental Life," Simmel argues that strict monetary transactions, as opposed to something like a barter system, flattens social relationships by removing the personal and emotional elements from transactions. Monetary exchange, he contends, encourages calculation and impersonal obligation on behalf of its participants.

As a result, social interaction increasingly requires quantitative as opposed to qualitative analysis. Writing about the homogenizing process of money, Simmel explains that fixed pricing "reduces all quality and individuality to a purely quantitative level. All emotional relationships between persons rest on their individuality, whereas intellectual relationships deal with persons as with numbers, that is, as with elements which, in themselves, are different, but which are of interest only insofar as they offer something objectively perceivable" (12). ${ }^{24}$ For Simmel, rationalization is one of the central tenets of modern life, and monetary exchange is an expression of rational value judgments. Video games are defined by similar goal-oriented decision-making processes, and certain critical games scholars have noted the continued privileging of rationalization and efficiency in contemporary game design. Pedercini notes that, "if computer games, in

\footnotetext{
24 methods" (12).

Simmel's use of the term intellectual here refers to being "understood by purely rational
} 
their immense variety, have anything in common, [it] may be their compulsion for efficiency and control. Computer games are the aesthetic form of rationalization" ("Videogames and the Spirit of Capitalism" n. pag.). ${ }^{25}$ Following Simmel's argument, the auction house homogenizes the process through which players can potentially interact, thereby limiting the decisions for players to make meaningful decisions and experience meaningful interactions with other players.

In my introduction, for example, I describe the early macros employed by players trading independently or using auction houses in World of Warcraft. These macros are premised upon the idea that trade is an optimized form of informational management as opposed to social interaction because trade is programmed specifically as a non-diegetic operator moment. Moreover, macros were eventually built into the game, and updates allowed players to enchant items and sell them directly through the auction house, thereby removing another social element of trading. As a result, players are encouraged to view trading as nothing more than what is objectively and quantifiably exchanged. Lehdonvirta explains that "social relations are central for deriving value from [digital] goods. The bits that constitute virtual goods have little experiential value without meanings attached to them within a social world. The prerequisites of social control are therefore in principle present when virtual goods are consumed, but the question is what the nature of the social world is like: caring or indifferent" (n. pag.). Interfaced auction houses remove a large part of possible social relations from trade, however, and produce

Echoing Simmel over one hundred years later, Pedercini, with some influence from Fredric Jameson, contends that despite the factory's virtual disappearance from Western life, consumption and production remain governed by the logic of rationalization. Food, for example, is stripped of its cultural specificity, education is determined by standardization, and "even our daily relationships are efficiently managed through online social networks which impose standard protocols and numeric rankings on previously irreducible social interactions." 
indifferent consumers and producers. They accomplish this in part by undermining the potential emergent and interpersonal relationships possible from trade between one player and another. Instead of two player-characters engaging in trade, trade windows facilitate trade between two individual players, thereby separating the diegetic world of the game from the player, stripping histories from items being exchanged, and denying the productive, dynamic stories players could have told about the relationships they built.

Simmel's critique of the money economy in "The Metropolis and Mental Life" provides historical parallels to the social role of the auction house. Money, for Simmel, is also related to the division of labour. The division of labour is strictly programmed into World of Warcraft, evidenced by the different skill sets players can choose, and whose wares are then sold on the auction house. Writing about the metropolis as a central site for the division of labour, Simmel identifies that diverse, but dissimilar and impersonal, interests and skill sets are organized under fixed monetization. This division of labour is striking not only because it is arbitrary and intentionally designed into the game, but also because it is partially consolidated by interface operations. The loss of MPs, for example, indicates a shift in the division of labour in games like World of Warcraft. Similarly, the auction houses function as an organizing principle analogous to Simmel's description of fixed currency in the metropolis. Similar descriptions can be made of the Steam Market as well, although antagonistic forces are more apparent in that system. The flattening of social relationships into informatic principles that Simmel describes is documented in narrative form in Stephenson's Reamde, as well. 


\section{Chapter 3: Total Market Control}

In 2001, Edward Castranova asked if video games would become an important part of social life for humans. He was specifically concerned with economics, and in particular questioned how a "large emigration of work and play time to virtual worlds would affect the economy of the real world" (“On Virtual Economies" n. pag.). In retrospect it might appear as a myopic question. However, the particulars of how games affect social life and political economy are still only on the margins of critical game studies. While Castranova was never quite interested in the potential for exploitation, due in large part to his orthodox economic theory and positivism, Marxist game studies scholars often describe a correlation between capitalist ideology and game play. The leftist games critics surveyed in Chapter 1 are largely concerned with video games as sites of capitalist ideological and cultural (re)production. In this chapter, I explore the increasing control over multinational trade within video game markets. Reamde, for example, predicts the increasing interchange between digital and real currency. The institutional apparatuses that define the fictional world of T'Rain resemble the increasingly global nature of MMO and game economies. Moreover, the novel correctly identifies the manner in which play is directed in order to produce capital.

One of my goals throughout this chapter is to explore the dialectic between play and labour using the Steam marketplace as a case study. Steam is a meta-game, though, and not in the strategic sense. It is a massive conglomeration of information detailing and manipulating the relationship between games and market forces. Valve, Steam's parent 
company, might be described as a metonym for value, a single letter away from the purpose of its Market. It is subsumptive in that games are brought under its umbrella and rendered part of the Steam network, which constitutes much more than a game, publisher, developer, or distributor. The Steam Network operates as a technology to dismantle brick and mortar game stores by undercutting them and supplying a stock far beyond the capability of a physical store. Moreover, it has successfully monetized play through collectible cards, item drops, and other secondary games. Steam is the meta game that accumulates national currencies and swallows them whole.

\subsection{Reamde Predicts The Future}

Neal Stephenson's Reamde(2011) is an exploration of video game markets in a globalized economy. This drawn-out, turgid, and complex thriller navigates the real world and the virtual world of T'Rain, an MMO, as if they are seamlessly connected. Richard Forsythe, the novel's protagonist, designed the world of T'Rain to resemble the real world as closely as possible (42). The reason for this, Forsythe notes, is to foster a community of players who can hardly distinguish between T'Rain's mimetic reflection and the real world. Forsythe's motivations for creating T'Rain are two-fold: his of love of video games, and the need to launder money.

The novel begins by recounting Forsythe's youthful exodus from the mid-western United States to British Columbia, Canada. During this time, Forsythe worked as a guide for wealthy hunters. On one of his guiding trips, he happens upon an abandoned alcohol smuggling camp that he eventually uses to run marijuana across the border. In so doing, 
he acquires wealth that he uses to purchase land and a resort that begin to make legal profit on their own. Throughout this period, he suffers from extreme ennui and depression and spends the majority of the next decade playing video games. The thriller aspects of the novel begin when a virus, Reamde, hijacks various T'Rain accounts and holds them at ransom for money. As a result, a weaving and complicated narrative emerges involving jihadi terrorists, Russian hackers, far-right survivalists, tech-nerds, mid-west family relations, protests and tribal conflicts in T'Rain, jet-setting kidnappers, Chinese gold-farmers, and nauseatingly detailed gun battles.

The bloated descriptions of some of the novel's plot elements and action sequences have led to criticism. Hellstrom, for example, explains that the "very aspects that make Reamde a thriller are the ones that Stephenson's core fans reject.” This rejection is because the novel's plot moves from the concerns of geek culture - goldfarming, T'Rain, game culture, technological advancements - to "a complicated plot involving al-Qaida, survivalists, and many fetishist descriptions of guns.” These criticisms miss the point, however, that the novel is trying to make about embodiment and the virtual. When Forsythe constructs T-Rain, he hires people who obsessively construct its every detail, from its geography, geology, language, culture, and history. The hundreds of pages describing guns and gun battles serves to reiterate the increasingly tenuous boundaries between game play and real-life. Violence and money flow seamlessly between the virtual and the real in the novel. The virtual geological accuracy of T'Rain is paralleled by details in the offline world, as well. For example, the novel describes Forsythe's driving as follows: "On his way out of town, deeply preoccupied with the fact that ersatz wuasi-Elven race known as the K'Shetriae were now embedded . 
.. in the memory chips of real-world GPS systems, he almost plowed into the back of what passed for a traffic jam" (29). Additionally, Forsythe recognizes the necessity of programmed scarcity for MMO economies to function. Video games, he realized, mimicked this process: "gold pieces were reposited in dungeons guarded by monsters. The more powerful the monster, the more gold it was squatting on. To get the gold, you had to kill the monster, and building a character powerful enough to do so required a certain amount of time and effort" (35-36). Richard designed the virtual gold to stem "from the same basic geological process as in the real world. The same, that is, except that they'd be numerically simulated instead of actually happening" (36). The shared qualities of the novel and the game indicate the increasingly malleable relationships between the offline and online life.

Moreover, the novel's narrative movement from in-game concerns regarding, say, gold-farming, to broader global issues, parallels a similar movement among video games from trade affordances in World of Warcraft to the international esports gambling associated with Steam. Referencing these situations, Forsythe explains that:

Gold farming had been an unwelcome by-product, an epiphenomenon, of earlier games, which had done all that they could to suppress the practice, even to the point of getting the Chinese government to ban such transactions in 2009. But in Richard's opinion, any industry that was clocking between $\$ 1$ and $\$ 10$ billion a year deserved more respect. ... It was only necessary to structure the game's virtual economy around the certainty that gold farmers would colonize it in vast numbers. (35)

Forsythe convinces his partner Nolan that managing the economic market of an MMO is 
the structural requirement of its success. He explains that constructing the essential "plumbing" of the money flow system would allow every other aspect of the game to operate properly (34). Forsythe mentions that they pulled off the extraordinary feat of making an MMO that was as accessible to Chinese teenagers as it was to "the podgy middle-aged Westerners who were dependent upon those Chinese teenagers for virtual gold. ... Westerners got to have more fun, since they could purchase gold pieces and use that virtual cash to fund spectacular building projects and wars . . . But, on the other hand, those kids in China were actually making money" (35). Forsythe's account of gold farming recalls Yee's research on Western anxieties surrounding gold farming. Moreover, the novel accurately describes the ways in which MMO economies cross national and cultural boundaries.

Reamde crosses other boundaries as well, as it often attempts to blur the distinctions between the digital and the physical. The novel is gamic in that combat scenes are described in unambiguous information. Tanner Jupin (2014) contends that Stephenson's novel calls "attention to the dynamics of these narrative ecologies, bridging the gaps between print literature and digital media and games by incorporating narrative fragments from gamic worlds and situating gamic fictions as just additional nodes within these larger virtual world assemblages" (10). Even Forsythe's descriptions of travelling are gamic. The overlay of gamic and real-world economies, violence, tribalism, fashion, and cultures are blended together. It is no mistake that Forsythe's description of his family firing rifles at the beginning of the novel mirrors the familial relationships in T'Rain.

Similar to other MMOs and games, T'Rain contains cosmetic items and virtual 
goods available for real-money trading. Forsythe, explaining the early design of his game, recalls an interesting bit of T'Rain's item history:

it was necessary to give players some freedom in styling their characters, because they liked to express themselves and to show some individuality. So there was an interface for that. Your K'Shetriae cloak could be made of fabric in one color, fringed with a second color, and lined with a third. But all three of those colors had to be selected from a palette, and Diane had chosen the palettes. So in the game's early years, it had been easy to distinguish races and character types from a distance just by the colors that they wore.

Then someone had figured out that the palette system was hackable and had posted some third-party software giving players the ability to swap out Diane's official palettes for ones that they made up to suit their own tastes. Corporation 9592 had been slow to react, and so this had become quite popular and widely used. (48)

This fictional story parallels the development of an almost identical phenomena in Team Fortress 2, a Steam game. Mitch Bowman explains:

Valve has, on occasion in the past, been direct in its dealings with the trading community. In 2009, when a bug appeared that allowed TF2 items to be farmed while idling in a server, Valve patched the exploit out and awarded everyone who had not taken advantage of the bug an exclusive hat. It's called the Cheater's Lament and, when worn, looks like an angel's halo above the player's head. Many traders still own this item and feature it on their Steam profiles like a badge of honor. (n. pag) 
In both instances, skins are cosmetic items closely associated with virtual and non-virtual identity, thereby working to unite consumption across virtual and non-virtual experiences. Jen Martin (2012) explains that avatars are less likely to be governed by "physical or survival needs" than by consumption (Keeping Up with the Virtual Joneses 42). Martin explains that "the purchase of goods with which to outfit an avatar, especially within more social environments, is based largely on meeting social needs, or, relatedly, culturally-informed expression. In turn, it is these social needs that make possible exchange-value" (42).In both T'Rain and Steam, for example, virtual identities and skins are occasionally associated with personal bank accounts and information.

Despite its criticisms, the novel's plot is less relevant than the informational environment that Stephenson constructs. As Jupin explains, Reamde describes MMOs as part of a broader "media ecology, an infinite interconnection of coevolving medial nodes and social and economic transactions" (Gamic Fiction 6). ${ }^{26}$ The novel, according to Jupin, "fully interrogates the interconnection of the real and the gamic, working through the ideas of video game theorists Jane McGonigal and Ian Bogost concerning potential gamic interventions in real world interactions" (6). Central to the world of T'Rain is its construction of value through in-game purchases, item exchange, and trade. Unlike World of Warcraft, however, T'Rain does not attempt to delineate its economy from the real world economy. Instead, the amalgamation of currency, cultures, and players from various economic and social strata are widely incorporated in T'Rain. In this way, the novel marks a transitional moment in which in-game and real-world economies increasingly interact with one another, and gestures towards the development of

26 (2005). 
something like the Steam Market. The media ecology that Jupin describes is similarly described in ethical terms by Sicart as the infosphere.

Tanner correctly identifies that Forsythe's economic success is associated with his ability to mediate between worlds, and to successfully integrate technology at a rate faster than his peers (43). The result is that he is capable of exploiting, manipulating, and controlling information and currency before others can. This strategy is similar to what the Steam Market has successfully done: creating an API that promotes the integration of information across individual games and their associated economies. Amy Wending explains this integration as a technological feature necessary for the development of capital:

The more accumulated resources work to expand this scientific and technological infrastructure, the faster the capitalist's machine becomes a dinosaur. In this mode of production, speed is thus of the essence if the capitalist is to redeem as much use-value as possible from machines that have been purchased. Speed will be the key element to the maximization of profit. The products of a new technology are most valuable before the benefits of the new technology (i.e. its increased production of material wealth) become generally applied to the production of a commodity, and employed by competitors in addition to the initial innovator. This results in the tendency to guard the secrets of technology rather than to generalize its benefits to humanity as a whole. It bears repeating: capital is interested in the increase of value, not in the expansion of material wealth. (107)

Reamde makes associations between security, technological development, and game play at various occasions. As previously mentioned, T'Rain's design required experts in 
diverse fields to create an MMO that was technically innovative and meticulously crafted. Jupin mentions how Reamde portrays Wikipedia as a negotiation of information and noise in the novel. Early in the novel, Forsythe acknowledges that he safeguards personal information about himself that is not on Wikipedia. This is relevant to the novel's construction of a mediasphere because of the association between medialities that the novel discusses, but also because it represents a disjunction between publicly available information and privately hosted data crucial to companies like Valve. Valve is a corporation whose public information and history is available in the form of release dates and game titles, but whose inner workings and economies are completely muddied by a host of missing details. Even Varoufakis's blog about the Steam economy only provides vague details and broad descriptions about its control and potential. For example, Forsythe describes his niece Zula's attempt to trace gold movement related to the REAMDE virus by tracking a player who had simply cast a healing spell on her character in an earlier play session (556). The major conflicts in the novel result from a botched exchange of information, the titular Reamde hack, and other incidents of hierarchical information management.

The novel highlights the manner in which video games provide users with satisfying experiences, but also demonstrates that these games exploit their user base in return, sometimes in unforeseen or unacknowledged ways, or sometimes by obvious means. Additionally, the novel highlights the growing legal issues surrounding MMOs, and their particular gray area with regards to government regulation and control. Stephenson indicates that national governments often find themselves inept at managing a global system such as an MMO. Forsythe develops T'Rain as an intentional source of 
labour after playing World of Warcraft and deciding it ought to accept its mimetic potential to reflect actual markets, and thus the novel also mirrors the manipulation of the Steam Market that Varoufakis recalls on his blog.

\subsection{Brokering the Steam Market}

In order to guide my discussion on work and labour in this section, I focus on the Steam Marketplace's relationship with the game Counter-Strike: Global Offensive. CS: $G O$ is an extremely popular esport that regularly fills traditional sporting arenas all over the world with spectators. These tournaments offer prizes and sponsorships in the hundreds of thousands. ${ }^{27}$ The rise of $C S: G O$ as an esport has been closely associated with the game's cosmetic items, which can be purchased and traded by users. Joshua Brustein and Eben Novy-Williams explain that $C S$ : $G O$ 's success was somewhat of a surprise: "it was the latest in the aging Counter-Strike series and came out at a time when there was no shortage of other shoot-'em-up games to choose from. Everything changed when Valve introduced something new: decorative virtual weapons, known as 'skins,' that could be acquired in the game and sold for real money" ("Virtual Weapons" n. pag.). Weapon skins are similar to cosmetic items available for purchase in other Steam games, and can range in price from a few cents to hundreds of dollars. Brustein and NovyWilliams report that "when it introduced the skins, [Steam] said in an announcement that the online arms bazaar would let Counter-Strike players 'experience all the thrills of

27

There are many other popular games released and managed by Steam, including DOTA 2, which have similar tournaments and prize structures. However, I chose to focus specifically on CS: GO because of the game's popularity, but also because of its well-documented trading practices, communities, and scandals. 
black-market weapons trafficking without any of the hanging around in darkened warehouses getting knifed to death"' (n. pag). They explain that this description was supposed to be a joke.

Modding has always been an important aspect of Counter-Strike culture, and might help explain many users adoption of weapon skins. In fact, the original version of the game was developed as a mod for Half-Life and was formally acquired by the Valve Corporation after it gained popularity (Wikipedia). Similarly, weapon skins are generally community generated mods by specialty graphic designers who are hoping to receive a portion of the skin's sales. Recall Varoufakis's comments that the Steam Market is designed as an entrepreneurial space in which savvy designers and artists could strike it rich ("To Truck, Barter, and Exchange?"). A recurring theme throughout Steam's business operations is the management of information and vectors through which information travels as opposed to the production of material objects. Like Uber, which owns no vehicles, and travel sites which own no hotels or planes, Steam derives value from being the service through which people acquire games. Even casters and commentators in the professional $C S$ : $G O$ scene are hired by secondary contractors. Professional CS: GO is unsurprisingly more complex than Brustein and NovyWilliams report. For example, some professional teams have existed with essentially the same four or five players since the mid-to-early 2000s. Moreover, the generic shoot-emup games they describe do not accurately reflect the play patters and influence of the Counter-Strike series as a whole. Brustein and Novy-Williams are mostly correct about the correlation between esports and the rise of cosmetic items. With the growth of major $C S: G O$ tournaments, Steam released virtual capsules and crates that could be unlocked 
with the Steam Keys, one of the community currencies mentioned earlier. Player names, team names, and other stickers or specialty cosmetic weapon skins could be opened in these tournament cases, and they are generally the only items worth upwards of $\$ 500$ USD.

The professional circuit is equally self-managed and -operated as far as the majority of leagues and tournaments are concerned, despite its reliance on Steam for game updates or patches. On a few occasions, professional play has come into conflict with Steam over patch or rule changes. In particular, these conflicts are the result of Steam developers attempting to appease the general public who have an obviously different perspective and play experience. For example, at one point the AWP, a oneshot kill sniper rifle with extremely poor mobility and a high skill ceiling, was nerfed ${ }^{28}$ due to popular complaints about "camping" by the general public. In competitive play, the AWP only saw play, if at all, among one dedicated specialist on a team of five. Yet, players who were exceptional with it could be devastating. Humorously, the nerf resulted in the top player in the world at the time, French player and dedicated AWPer Kenneth Schrub, becoming an average-ranked pro player overnight. Duncan Shields, a selfdescribed amateur esports historian and professional esports caster, noted that, as a result of the nerf, prices of stickers or weapon skins bearing Schrub's name dropped significantly in value on the Steam market ("The Awp Nerf" 6:00-7:00). While gambling may have contributed to the rise of $C S: G O$ as an esport, the professional scene now also influences the trade market.

Professional players have, at times, commented on their discomfort with 
developers and other non-professional players dictating the terms of their game. For example, in early 2016 a patch released a new gun (a new way to market different skins), that was so wildly unbalanced it resulted in numerous league events and tournaments being cancelled or run with the pre-existing patches of the game ("The CZ Effect" $n$. pag). More troubling, there have been cases of players being summarily and publicly fired for discussing unionization in order to combat poor treatment by tournament organizers, some of whom are subcontracted by Valve. In the winter of 2016, for example, Sean Gares and numerous other well-known pros published a letter regarding players' rights, and were promptly released from their team contracts ("Pro CS:GO players issue open letter" n. pag.).

Most professional $C S$ : $G O$ teams are operated by a professional esports organization, and are additionally sponsored by hardware and software companies, including Intel, BenQ, and Razor. Although Steam does not pay players personally, contemporary top-tier teams make extraordinary amounts of money. Professional Polish team Virtus.Pro recently revealed in an interview with Anton Cheryepennikov that each of their players received approximately $\$ 20,000$ a month in salary prior to their supplementary sponsorships (rnsonline). At any rate, Steam also benefits from the existence of the professional circuit despite its passive involvement in it. By owning both the game and cosmetic items associated with it, the growth of the game as an esport concomitantly drives Steam profits. As noted in the introduction to Steam previously, all transactions are taxed, so to speak, and any exchanges or sales of $C S$ : $G O$ skins contributes to the market's growth.

Despite Steam's intention, gambling was ultimately a more powerful driving 
force behind skin sales than its player base's alleged black-ops fantasies. The introduction of skins brought about an enormous market for gambling websites intent on profiting from the real-world value of item trading. With access to a market capable of liquidating digital items for cash, the Steam market exploded with people buying skins for cash, then using those skins to place online bets on professional CS: GO matches. The game, due in no small part to its increased market presence, skyrocketed in popularity. In mid-2016, Brustein and Novy-Williams reported that the game has sold over 21 million copies, while bringing in $\$ 2.3$ billion dollars across 3 million unique gamblers. In addition to driving sales for the game, esports gambling is also taxed by Steam's Marketplace. The Market has astonishing levels of internal security to ensure the safety of exchange between players. One result of this security, however, was that gambling sites required users to register their accounts (and therefore their items and skins), thereby ensuring the full payment of any gambling results. Because the gambling sites are run on the built-in software provided by the Marketplace, Steam collects 15 percent on all of these transactions. All of this economic activity generated capital for Valve, and $C S$ : $G O$ is not even Steam's most popular game, although that might partially be due to its pay-wall. DOTA 2, the world's most popular multiplayer online battle arena (MOBA), is also owned by Steam, and contains an active and growing esports scene as well. Moreover, DOTA was also originally a mod for Blizzard's Warcraft III: Reign of Chaos.

The professional CS: $G O$ scene is inextricably linked with trading, gambling, and real-money transactions. Steam is committed to fostering communities of dedicated players. During major tournaments, for example, players can receive specialty cases and 
skins only available to those watching the tournament through their Steam client. Steam is able to consistently accumulate labour value by encouraging growth within its user base. The constant source of revenue accumulation - the source of labour in the Steam marketplace - is its community. I am specifically interested in how the communities surrounding $C S$ : $G O$, not the game itself, are the sites of labour. Generally speaking, games are sold as a one-time commodity into which players can invest any amount of hours of play. The new model for games - think mobile apps, in-app purchases, and especially the Steam model mentioned above - try to monetize the player-base after release. The message boards, Twitch streams, esports, gambling sites, and other activities supporting a game like $C S$ : $G O$ are the actual sources of exploitative labour value for Steam. Work free from alienation is impossible within the Steam marketplace because the affordances, interface designs, and community exchange practices are guided by principles of exchange value.

Stephanie Boluk's ongoing research focuses on this massively profitable operation, and attempts "to understand economic experiments that move away from fun or entertainment and converts play into a new, form of abstraction: productivity" ("Current Projects" n. pag.). What Boluk refers to as the loss of "fun" equates to the loss of social cohesion through the abstraction of intersubjectivities. As it pertains to video game play, free or playtime in the digital era is constantly financialized, be it through direct purchase or unwilling labour via data management and accumulation. As for the lack of social cohesion, I am referring specifically to the increasingly automated management systems that govern the processes of online trade, and thereby reduce social interaction and generative diegetic properties that can potentially emerge through the act 
of trade. Boluk's intervention is largely concerned with immaterial labour and its process as a whole, but provides insight into trade relationships as well. Interestingly, Boluk's concern is largely one that Wark's Hacker Manifesto (2004) is concerned with: the ownership of the channels through which information travels. The means of dissemination and communication are what is relevant here, and take the place of the means of production.

While Boluk is correct in highlighting the abstraction of play into productivity, Steam provides an environment in which non-play is abstracted into exchange-value for Steam. When I refer to Steam as a metagame, I am referring to its ability to subsume play along with other activities into forms of value. Varoufakis's describes, for example, the various in-game economies that operate within the Steam Market. Additionally, recall his point that one of Valve's goals for the Steam Market was to "blend together different gaming interfaces." There are, on Steam, various games that produce in-game economies and manage them in their own distinct way. Team Fortress 2 (TF2), for example, is mentioned in passing a few times throughout this dissertation because of its distinct formation of currencies, including cosmetic earbuds or cosmetic hats, which mark it as a particularly powerful site of user-generated economy. Other Steam games have economies made up entirely of gifts and friendly exchanges, while others still have trading cards or other collectibles users trade for without the use of real-money transactions (VALVEnomics). Even Recettear, the satirical trading simulation game I discuss in the following section, is released on Steam. Steam subsumes all these different manifestations of trade under its umbrella, creating an economic system that amalgamates and determines the economic potential of any game it publishes. Any developer who 
wants to see their game's economy or virtual items represented on the Market must also abide by the broader restrictions established by Steam.

Furthermore, the "astute traders" that Varoufakis refers to have since arrived in the Steam Market, and they do not represent the entrepreneurs in search of economic equilibrium and self-realization that he had hoped. As mentioned previously in the discussion of gambling sites, the Steam API allows users to download other user's inventories and arrange the data to produce statistics about, say, the origin of an account by checking its IP. Mitch Bowman notes that the majority of third-party real-money transactions for TF2 earbuds in 2012 were from Russian IPs (whether actual or misdirected). While the Reamde discussion might lead one to assume that Steam encourages a network of money laundering, it actually indicates a much more pedestrian practice: credit card fraud. On occasion, Bowman explains, Steam goes after some of these accounts, but many of them are able to convert the items to cash before Steam can handle the complaints. Furthermore, Bowman notes, various traders have worked at times to artificially inflate the price of items used as currency, such as earbuds, in $T F 2$.

Despite any ethical concerns they purport to have, Steam stands to benefit from the majority of trades that take place within their system because of their trade restrictions which force players in search of cash to turn towards third-party sites. As a result, Steam can, and has on numerous occasions, explained to players that any loss on their behalf was cleared multiple times through Steam Guard, the Market, and other security measures. Steam has positioned itself in a way so as to benefit from the very participation in its economic system, much in the way Jodi Dean's Blog Theory (2010) viewed communicative capitalism's relationship to blogging and other social media 
activity.

Writing about digital labour, and specifically digital labour's function on social networking sites like Facebook, Christian Fuchs and Sebastian Sevignani contend that communication and information is a form of labour that is exploited under capitalism. They note that while "others have stressed that social media enable participatory culture" or maker culture, "the debate can be interpreted with the help of Marx's analysis of the dual character of labour as concrete work that produces use-values and abstract labour that generates value" ("What is Digital Labour?" 254). Fuchs and Sevignani define labour as

a necessarily alienated form of work, in which humans do not control and own the means and results of production. It is a historic form of the organisation of work in class societies. Work in contrast is a much more general concept common to all societies. It is a process in which humans in social relations make use of technologies in order to transform nature, culture and society in such a way that goods and services are created that satisfy human needs. (240)

The distinction between work and labour that Fuchs and Sevignani employ to critique social media is a productive lens for addressing the exploitative practices found in online video game communities. Under this distinction, work and play form use values, while labour and play form exchange values. They explain that "Marx distinguishes between an anthropological and a historical quality of collective activities that satisfy human needs: work and labour. This distinction is reflected in capitalism in the dual character of the commodity that is both use-value and (exchange) value at the same time" (240). Moving forward, I consider how to identify the sites of (alienated) labour versus the sites 
of (non-alienated) work in video game trade spaces. By doing so, I hope to examine the capacity, if any, for a game play of work within an otherwise exploitative labour space. Labour, under Fuchs and Sevignani's interpretation of Marx, is always alienated, while work is not.

Media studies critics (Dean, Dyer-Witherford, Mosco, Terranova) have noted that immaterial or affective labour is a central quality of contemporary digital social life, but not its specific function in gaming. First, it is important to remind ourselves that communication is an inherently productive process - it is work. The processes of communication and information gathering require different types of work. Drawing on Raymond Williams's descriptions of communicative work, Fuchs describes work as cognitive, communicative, and physical. It is within the communities of players that labour takes place, and not individual play patterns.

In Marxist economics, the social sphere is essential to the creation of value. Michael Heinrich explains that in order to "behave toward things as commodities, that is, to relate things to each other as values, the owners of commodities must relate their commodities to a general equivalent" (63). The Steam Marketplace generates two equivalent commodities for users to exchange items, which I have noted above. First is the ability to use real-world currency for Marketplace purchases, and second is the community use of Steam Keys as a form of currency among traders. The Steam Market creates market value by introducing its own currency as a point of possible exchange among its users. In so doing, they are able to fix commodity prices and exchange values. The exchange value - that is, the value users place on their skins or keys - is largely irrelevant to the method by which Steam accrues value from its user. Any in-game items 
that players acquire, regardless of how each player values these items individually, are irrelevant when flattened by the Market's general equivalents. The use of Steam Wallets and Steam Keys in the Marketplace is an important lesson in understanding general equivalents of value as well. Money is a form of value created, according to Heinrich's reading of Marx, only at the moment in which "social character of value expresses itself in a specifically social form of value" (61). That is to say, Steam Keys and Steam Wallet currency are only viable as currency forms if the community abstracts the other products of their play.

The value of the market is derived not from the individual labour of its users, however, but through the very act of exchange among them through fixed rates and "general equivalents." It is helpful to think of the Steam Market in this regard as operating in the logic of financial derivatives. Robert T. Tally Jr. explains derivatives as follows: "At the risk of oversimplifying matters unduly, one could define a derivative as any contract whose value is derived from something else, an underlying asset or an index, for instance. Indeed, that is all a derivative is, yet, like the commodity, it turns out to be far more complicated than a mere item used and exchanged" ("Meta-Capital" 3). A simple example of a derivative might be a farmer who agrees to sell wheat at a particular price to a bakery supplier. The future price of wheat might go up or down in the future, but the contract is settled in the present. According to Dick Bryan and Michael Rafferty's Capitalism with Derivatives (2006), derivatives tie the future to the past through flexible market, malleable labour, and floating exchange rates (103). Additionally, derivatives acquire value through social relationships, similar to the process under which Marx described commodities acquiring values. The difference, however, is 
that derivatives are viewed simultaneously as financial assets and liabilities, and, according to Bryan and Rafferty, exist as “objects of speculation, somehow outside of money" (135). For Bryan and Rafferty, derivatives represent the financial system that has replaced gold as the global financial anchor (105). The shift toward financial derivatives as a global anchor has not been established intentionally through national or international regulation, but rather "a movement created within the market; by capital for capital" (133). Derivatives constitute the logical conclusion of globalised capitalism by providing a system of networked anchors. The market requires derivatives specifically because they are flexible, and thereby allow the global financial system to operate "as if" it were stable (134). Tally provides a description of how derivatives bind the globalised economy:

For example, a share of stock represents real ownership of part of a company, and a bond represents ownership of a quantity of money, but a derivative need not involve actual ownership at all. Rather, the derivative is meta-capital inasmuch as it functions to bind together and to blend other forms of capital. Derivatives allow one to compare two otherwise separate and distinct kinds of assets (fixed versus floating interest rates, for instance), and they can be used to create hybrid instruments that will tie different assets together. ("Meta-Capital" 8)

The Steam Market operates in a similar capacity in that its labour force, capital, and assets are bound within a hybrid state ( $C S: G O$ skins, for example). Moreover, the source of capital and its assets are located outside of the corporate entity that produces them. In this regard, derivatives provide an avenue of potential resistance. As its profit exists in a liminal space operated by shifting groups of labourers, the inherent instability 
of derivative-based markets can potentially enable radical change. Although the instability of derivatives is what Bryan, Rafferty, and Tally associate with the financial crises of globalised capitalism, Autonomist Marxism might view this instability as a site of possible resistance. The forthcoming discussion on the iBuyPower banning and shifting public opinion on $C S$ : $G O$ skin gambling is indicative of this possible resistance.

The Steam Market abstracts labour from its user base through social efforts as a whole. While Adam Smith might have theorized the economy to begin with the isolated, rational individual, Heinrich considers the "social relations in which the individuals are embedded at any given time" (46). Interestingly, Heinrich notes that individuals must conform to trade practices to operate within a specific social setting in order to function. Users do not have alternative methods of trade other than gift, which still relies on the affordances Steam provides. The function of the Steam Marketplace, then, is to convert concrete labour (play and work) into abstract labour by commodifying its community.

I consider play a form of concrete labour, or even what Fuchs and Sevignani refer to as work. For Marx, concrete labour in and of itself did not produce exchange-value. Play is, as Marx defines work above, a term that refers more to the generalized activity in which humans engage. Heinrich notes, however, that value "is not constituted by a particular concrete labour or through a particular aspect of concrete labour. Every act of labor whose product . . . is exchanged produces value. As values, the commodities are qualitatively equal" (48). The act of play is a form of concrete labour that only derives value through its abstraction and exchange. The abstraction of play in the Steam Marketplace is the assignment of equivalents and values to the by-products of play's concrete work. Heinrich clarifies that "in the case of abstract labor, we are not dealing 
with such a 'mental abstraction' but with a 'real abstraction,' by which we mean an abstraction that is carried out in the actual behavior of humans, regardless of whether they are aware of it" (49). To understand Heinrich's point about abstract labour, it is necessary to recall Marx's insistence that exchange is a fundamentally social expression. Exchanging items is not, then, an individual act, but one that requires a host of social affordances that underscore and overwrite the actions of each person involved in the exchange. Marx explains that value is produced through the process of exchange. As Heinrich notes, "the abstraction really occurs, independent of what the participating commodity owners think" (50). Marx insists that the act of exchange is precisely the site of abstracted labour: "the reduction of various concrete private acts of labor to this abstraction of equal human labor is only carried out through exchange, which in fact equates products of different acts of labor with each other" (50, qtd in Heinrich). Thus, the value of digital items in the Steam Market place are the abstraction of play, and exist within the social sphere of affordances allowed by the Steam Market.

When I began my research for this paper, I had hoped to find some model of alternative forms of play, or perhaps patterns of resistance among the community of Steam users. On Varoufakis's blog, he praised the company's "anarcho-syndicalist" work environment, and, interestingly, celebrated the purported gift economies and barter systems found throughout Steam's marketplace and communities. At the same time, Varoufakis's blog celebrates the absolute control Steam has over its marketplace. Valve can manipulate literally every single point of data in the marketplace to bend it to their will ("Arbitrage and Equilibrium" n. pag.). The powerful design affordances Varoufakis refers to reiterate the power of trade interfaces and affordances which I have mentioned 
elsewhere. Unlike the emergent communities that Celia Peace (2009) and Manuel DeLanda (2009) write about, Steam's community is intentionally engineered and organized in order to abstract play into exchange-value. The security of the Steam Marketplace, which I mention above, is paramount to controlling potentially emergent communities. Emergent communities are, according to Pearce and DeLanda, inherently turbulent. Although design affordances contribute to the cultural developments of these communities, the resulting communities are meant to form varying intelligences, customs, and collectives. Whereas video games are often imagined - even by Varoufakis, ironically - to produce unintentional consequences and disparate play patterns, Steam attempts to bind its community by the same logic of capitalist neoliberalism that drives the real world.

Steam's absorption of alternative markets, such as barter and gift economies, the esports scene, and modding culture, signifies the consumptive powers of market driven exchange-values. For Pierre Bourdieu, for example, alternative or deviant social groups, cultures, fashion, or art are always swept up by market powers and sold back to the mainstream, creating a reiterative loop in which the avant-garde consistently produces new materials and ideas for eventual consumption under capitalism. The cycle of appropriation that Bourdieu describes plays out in the story of the iBuyPower banning and subsequent community reaction that I discuss below. The magnitude of CS: GO's position in the Market disrupts the potential for Steam users to allay its influence over indie games, prices, and availability of items, particularly when Keys function as the primary medium of exchange. Although I had hoped the gift exchanges, modders, or role-players might demonstrate an alternative, I conclude that only minor exchanges and 
moments eluded being abstracted into profit.

Nevertheless, the professional $C S: G O$ scene represents the capacity for Steam to create a labour-power capable of undermining their control. Video games that continuously siphon profit from the labour of its players, however, have an interesting capacity to move away from this exploitation. The continued success of the Steam Market requires that the community invest itself into a game like $C S: G O$ in accordance with their restrictions. Moreover, the Steam Market's profit margins are driven by exchanges of secondary items and cosmetics, and not the existence of, for example, CS: $G O$ as an esport. If Steam produces labour-power through its community, perhaps resistance or insight might be located by turning towards those in exile from official Steam tournaments. Recalling the insistence of Autonomist Marxism that labourers (players, in this case) be considered first in their relationship to Capital, I return to the case of the iBuyPower team permanently banned from official $C S$ : $G O$ tournaments for match-fixing in early 2015.

These exiled community members offer critique of the Steam Market, and, additionally, demonstrate the existence of a player base whose support lies outside of official channels. Of the banned players, Braxton "Swag" Pierce, Joshua "steel” Nissan, and Sam "DaZeD" Marine have remained active members of the unofficial CS: GO community. All three stream regularly on Twitch, have filled in as substitutes for professional teams, and have done commentary work in unofficial Valve tournaments. Despite their official banning, community members, secondary sponsors, and unofficial tournament organizers have provided these players with various $C S$ : $G O$ related work because they are regarded as experts in their field (Liquipedia). I am not attempting to 
argue that the iBuyPower team are necessarily virtuous or even likable figures, but that their continued involvement in the community represents the power of players, professional and casual alike, to reject or protest any game's official status quo. ${ }^{29}$ What follows explores the possibility of imagining these exiled players as constructing what might be referred to as a socialised player. Socialised players, similar to the socialised workers described by Autonomists, comprehend the system of capital in which they maneuver, and are capable of reappropriating the means of communication.

In the wake of the gambling controversy in the summer of 2016, various community members expressed outrage about gambling sites and the players they sponsored. These criticisms, recall, were mirrored in official press releases by Steam. They failed to address the overarching structural problems that enable these operations. Looking towards the exiled community, and Sam Marine in particular, there is another critique that arose. In a video discussing his banning in relation to the gambling controversy, Marine reflects on a variety of ironies and contradictions within Steam's official response. Moreover, Marine contends that the moral panic expressed by some members of the community is misguided and petty. Greed among entrepreneurs, Marine explains, is not the singular problem in this situation, and to become outraged over their behaviour misses the complexity of the situation. Dyer-Witherford explains that, typically, "Western Marxisms have tended to emphasise only the dominant and

29

The three players remaining in the game, Pierce, Nissan, and Marine, are difficult personalities. They fall prey to some of the worst stereotypical gamer behaviours, including sexism or homophobia, but that does not discount their representational ability with regards to critiquing Valve and the Steam Market. Marine has, on his stream, and even in the video cited in this section, employed ableist and homophobic language. The labouring classes have, however, always demonstrated heterogeneous opinions that do not always align with all causes on the political or social left. Marine's position within the community demonstrates the revolutionary potential of labour despite the potentially problematic politics among its populace. 
inexorable logic of capital, to a degree such that its accumulative logic, unfolding according to ineluctable (even if finally self-destructive) laws, figures as the unilateral force shaping the contemporary world" (132). In Dyer-Witherford's assessments, these particular forms of Marxism tend to minimize the complexity that defines the relationship between labour and capital. For example, Marcuse's construction of the "one dimensional" man asserts that people are guided by ideological forces which produce perfunctory desires and goals. Following this prototypical critique of mass culture, the community response indicates a belief that these companies are a representation of greed and desire that constitutes the one dimensional man.

However, Dyer-Witherford explains that Autonomist Marxism performs an inversion of this process, and centralizes labourers as "the active subject of production" (132, emphasis in original). Reflecting a similar proposition, Marine issues a similar critique of Steam's exploitation of its player base. In response to some members of the Twitch and CS: GO community, Marine explains that gambling sites and players or streamers who fail to reveal their stake in the company while promoting these is a minuscule problem:

It's gambling for kids? If you think [gambling sites] are gambling for kids, how much money does Valve make off gambling? If you don't think opening cases is the most rate form of gambling in the entire world you are ... a piece of shit. When you open a case you make an average of like 20 cents on the dollar ... That's like having house odds at 80 percent. How come you're not mad at Valve for giving you back $20 \%$ on the dollar? Opening cases is the worst, most shadiest, most fucked up gambling ever. Why don't you guys hate Valve? I don't 
get it. ("DaZeD on Valve" 0:00-0:40)

He explains that a gambling site will often have an edge of $6 \%$, meaning that an average $\$ 100$ bet would result in an average $\$ 6$ loss, while Steam has an $80 \%$ edge on the value of the cosmetic skins in their cases. Marine argues that blaming gambling sites, as many government officials, journalists, and Valve themselves are doing, approaches the controversy myopically by failing to address the structural inequity already in place. According to Marine, professional players and gambling sites, however corrupt they might seem, pale in comparison to the exploitation of the entire Steam Market's $C S: G O$ user base. Moreover, these sites provide players a space where percentages and statistics are openly broadcast, while Steam's $C S: G O$ cases disguise their nature as a gambling tool by masking it as a game. Marine identifies the manner in which the Steam Market employs video games as a form of creating capital through play, unwittingly reiterating the Marxist critiques from earlier in this section.

Following the controversy and Marine's reaction, public opinion on the banning of the iBuyPower team has shifted slightly. Richard Lewis, a renowned esports commentator and journalist, who wrote the article that eventually resulted in the banning of the iBuyPower team, has recently (April 2017) expressed his belief that Pierce's lifetime ban is unfair and should be reconsidered. In this particular vlog post, he addresses the recent meme \#FreeSwag (referring to Pierce's gaming handle), which frequently appears in Twitch chat during major tournaments and other CS: GO games ("The Absurdity of the \#FreeSwag Movement" 1:00-2:30). Moreover, all three players have been asked within the last year to perform guest commentary on or coaching work for unofficial Valve tournaments, indicating a shift in public perception with regards to 
their match-fixing. I credit this shift in public sentiment to the criticisms issued by Marine, Duncan Shields, and other prominent figures within the $C S$ : $G O$ community regarding Valve's draconic behaviour towards their player-base. This interpretation stems from Dyer-Witherford's insistence that critique must begin with "struggles: how they arise, how they are connected or divided, and their relation or lack of relation to 'official' workers' organisations, and their capacity to subvert capitalist command" (133). An Autonomist approach to the professional esports community, particularly its relationship to skin gambling, must begin by viewing the producers of value as antagonistic subjects who desire to express their own identities.

The iBuyPower team, then, represents a struggle for three excommunicated, socialised players who have remained to challenge the authority of the Steam Market and Valve's official decisions, and to sway public opinion, thereby representing a nascent political struggle or awakening among the esports population of viewers and players. The player communities, including professionals and casuals, as well as some journalists and other esports figures, have reappropriated certain aspects of the game from Valve. Twitch, for example, an immensely popular live-streaming service that broadcasts numerous Valve-related games and tournaments, from $C S$ : GO to Dota 2, is simultaneously propped up by the active producers of content and value: its streamers and viewers. Although Twitch has towed the party-line for Valve with regards to gambling sites, issuing a warning about their Terms of Service, many popular streamers, broadcasters, and viewers continue to openly gamble and bet on professional matches. I am not arguing that esports or skin gambling is a revolutionary act, but the community's resistance to official Steam Market policies, and the continued existence of these cultural 
practices, indicates the power in communities that construct economic value. Steam alienating its community base results in a loss of their source of labour, after all. That Marine's scathing critique of Valve and, to a lesser extent, Twitch came via his own Twitch channel is indicative of the socialised players' ability to reappropriate the means of communication they helped create. Similar alternative networks and design strategies have appeared in other unusual forms as well, and this next section provides a close reading of two indie games, while gesturing towards other sites of alternative trade, cultural, and social practices that do not readily comply with the stereotypical, Frankfurt School's critique of mass media. 


\section{Chapter 4:}

\section{The Avant-Garde, the Monetization of Play, and Evil}

In recent years, a few indie games have explicitly critiqued the growing relationship between labour and play. Recettear (2007)and Papers, Please (2013) are games that obfuscate the boundaries between labour and play in order to comment on the growing relationship between gaming, governance, economics, and social relations. These games produce play patterns that intentionally resemble menial office tasks and everyday working conditions. Additionally, these games carry a retro aesthetic that hearkens back to earlier video game design. Recettear, for example, is largely inspired by Super Nintendo era RGPs such as Mario RPG, Zelda: Link to the Past, and Earthbound. By revisiting this aesthetic, these games call upon a nostalgia for a time where play was not subsumed by online systems of trade and exchange. Nevertheless, these two games are deeply concerned with markets and exchange. Papers, Please can be read as a political unconscious about trade through its alienating conversion of play into a form of labour. Additionally, this section examines the 
theoretical implications of the allegorithm, as imagined by Alexander Galloway and McKenzie Wark, in relation to these two satirical games.

As mentioned previously, various scholars have written and continue to write about the growing infusion of labour and playtime. Although single-player games do not possess the vast economies and possible emergent cultures found within MMOs, they often reflect the same ideologies as their multiplayer counterparts. Unlike MMOs, however, single-player games can critique the processes under which games mimic ideological processes. These two games provide a consolidated critique of capitalism's encroachment upon both social life and game play. I chose these games as objects of study for their commentary on matters of political economy and trade, but also because of their self-reflexive criticism on the banality of game play itself.

Despite their critique, these games also take the very idea of play as a form of labour and attempt to invert it and make it enjoyable for players. Like other cultural producers within the avant-garde, indie game developers are critical of the corporate aesthetics that dominate their art form. Defining an indie game, however, is a difficult task. As Bart Simon (2013) explains, indie games are not a genre, such as an RPG, MMO, or platformer, and any formal definition will fail to account for the vagaries and qualities of indie games ("Indie Eh? Some kind of Game Studies" 2). "To speak of indie games," Simon explains, "is not to speak only of the games themselves or of the experiences of game play but rather of the cultures of game development from whence they came" (2). Phil Fish, one of the most prominent indie game developers and the designer of indie success $F e z$, explains in the documentary Indie Game that the goal of game designers is "to find new ways to communicate with other people." In a Kotaku 
article about Fish and the rise of indie game design, Fish is painted as an artist more than a game designer. The article introduces Fish as the "son of art school dropouts ... Fish had art in his blood" ("Going Indie" n. pag.). Fish is the prototypical indie game designer: he was fired from Ubisoft, a triple A game developer, and moved to making independent games. Reflecting on his time in the corporate game complex, Fish explains that "the way these people make games, it's so horrible. Hundreds of people on your team, you don't know any of their names. It's so big and impersonal" ("Going Indie" n. pag.). Additionally, Kotaku article notes that these developers are interested in the nostalgia of 1990s-era video games. At the edges of cultural production and perhaps incapable of moving towards new spaces, indie game developers have returned to the centre of capitalism to find inspiration for their games.

A combination of nostalgia and critique of contemporary game design is central to many indie developers. These games share thematic concerns often found in avant-garde art: an anxiety regarding the homogeny of society and cultural production, a distrust of bourgeois culture, a tenuous relationship with politics, and finally, the deception, often in a legitimizing manner, of society's castoffs. In James Swirsky and Lisanne Pajot's documentary Indie Game (2012), several designers describe their games as a reaction to the progressive drone of a homogenizing culture. Indie games, however, are always or often explicitly critical of the structures of late capitalism and its incorporation into the spaces of play. Recettear and Papers, Please, however, are notable exceptions because of their satirical commentary on the spaces of exchange and play in videogames. These games took the very idea of play as a form of labour and tried to invert it, to make labour a playful experience. They demonstrate what Terry Harpold calls a "nervous atavism" of 
backwards glances towards medial shifts and appearances (Ex-foliations 131). Harpold writes about repurposing, reusing, and replaying the same games in different methods in order to reinterpret the presupposed political implications of standardized game play. In an attempt to revisit or exfoliate the play patterns in historic RGPs, Recettear and Papers, Please attempt to reconfigure play to demonstrate, criticize, and destabilize relationships between labour and play so often associated with contemporary games. The criticism levelled against triple A and mainstream video games by indie developers is matched by academic theorists as well.

More so than their online counterparts, these single player games represent what Wark and Galloway refer to as allegorithmic games. Allegorithm is a portmanteau of allegory and algorithm. Broadly speaking, digital games are virtual simulations or representations of the neoliberal and capitalist logic that manifests in the real world. As mentioned in my previous chapter on trade windows in World of Warcraft, the algorithm of the game contributes to an allegory about social life that any particular player is rewarded for understanding. The social structure of gold farming, for example, is an allegorithmic process by which companies or actors understand the benefits and processes of trading within the game and reproduce capitalist social structures within their play. The algorithm is therefore an allegory of capitalist process. The coding of the trade window, for example, encourages a particular play pattern. To play and win a game, then, is to succeed at an allegorithmic level. A game like World of Warcraft, however, does not demonstrate its allegorithm openly to the player. Instead, players are rewarded for learning and understanding these systems. In Papers, Please and Recettear, however, the allegorithm is openly represented in order to satirize its existence. 
For Galloway, video games manifest a social order that is predominantly influenced by contemporary capitalism. In Gaming, he argues that videogames encourage users to internalize and understand a system of rules and code in order to achieve informatic power. Influenced by Deleuze's theory of societies of control, Galloway argues that system analysis and mathematics are the ideological umbrella under which contemporary biopower and capitalism operate. In "Realism and Post-Fordism," Galloway argues that the mathematical underpinnings of contemporary programming, such as set theory, are central to capitalist modes of production. He explains that "one cannot wish away the fact that the mode of production today favors software so greatly and that software is math. A simple syllogism reveals the conclusion that the mode of production today has a special relationship to mathematics. Software is thus the thorn in the side of contemporary philosophy" ("Realism and Post-Fordism” 359). Rules and patterns of play are internalized by players who are then capable of translating this information. To play a game is to interact with the information system and winning requires players to understand said system. Galloway's argument follows that players are thereby complicit in an ideological state of capitalism in most game play because to win means to enact "control allegories" in order to win (Gaming 83). Playing video games becomes, then, a representational form of the social order that allegorizes the current mode of production. In Papers, Please, for example, human life is quantifiable through bureaucratic processes, and Recettear imagines the possible life and death of an adventurer through the cost of doing business.

Problematically, Galloway's materialist critique of mathematics as an ideological structure fails to account for the social elements central to networked social life. 
Additionally, he tends to present players as passive consumers of ideology who are bound to reiterate the mathematical mode of production. Nevertheless, Galloway's distinction that video games, unlike other mediums, provide an aesthetic representation of social life under networked capitalism remains valuable. For this chapter, the essential takeaway from Galloway's allegorithmic readings is that video games are capable of representing systems of political control and social histories. Games offer insight into designers' and players' social response to and engagement with rules and systems of trade. The satire and critique inherent in these games demonstrates the flaw of Galloway's critique of code or math as an ideological formation and social driver.

Wark is less concerned with characterizing the formal properties of games than Galloway, and as a result his conception of allegorithmic space relies on abstracting the real world as a "gamespace." Similar to Galloway, Wark contends that the whole of social life is an imperfect reflection of video game logic. In a thoughtful reversal of assumptions, Wark argues that games provide idealized allegories for real-life situations. The world itself - gamespace - is a series of failed games while videogames are the embodiment of legitimate experiences:

No wonder digital games are the emergent cultural form of our time. The times have themselves become just a series of less and less perfect games ... . [G]ames [are presented] in a pure state, as a realm where justice — of a sort—reigns. The beginnings of a critical theory of games — a gamer theory — might lie not in holding games accountable as failed representations of the world, but quite the reverse. The world outside is a gamespace that appears as an imperfect form of the computer game. (25) 
Here, Wark is referring to the tension between capitalism's myth that any individual can succeed through determination and hard work, and overcome the gender, race, class, and other social inequalities fundamental to capitalism. Videogames, alternatively, offer "everything gamespace merely pretends to be: a fair fight, a level playing field, free competition" (25).

Like Galloway, Wark imagines the gamer as a victim of the vagaries of contemporary social life and allegorithmic game design. "The gamer's God," Wark explains, is a game designer [who] implants in everything a hidden algorithm." The gamer follows the structure of the game and internalizes its design: "All that is righteous wins; all that wins is righteous. To be a loser or a lamer is the mark of damnation. When you are a gamer, you are left with nothing to believe in but your own God-given abilities. Gamers confront each other in games of skill which reveal who has been chosen - chosen by the game as the one who has most fully internalized its algorithm" (11). While Galloway and Wark can be critiqued for their erasure of player choice, agency, or resistance, their failure to address user consumption is more of a rhetorical issue. Galloway and Wark construct players as (mostly) passive agents in order to underscore the magnitude of capitalism's influence on videogames.

Isolating the formal qualities of videogames can provide a space to address the contours of power that shape game design. Wark and Galloway's critiques tend to favour the pre-player texts, and, as a result, address the ideology laden within them. A brief example from Michel de Certeau's famous chapter "Walking in the City" will help illustrate the approach Galloway and Wark, as well as myself, sometimes take in order to discuss the pre-player text. In "Walking in the City," Michel de Certeau differentiates 
between strategies and tactics by positing them as oppositional as opposed to hierarchal practices. Strategies, he explains, exist in the realm of power and authority. Urban planning, for example, is a strategy by governments and officials to determine the routes and movements of citizens. De Certeau uses the World Trade Centre in New York City as a specific example of strategic construction designed with a panoptic view. Tactics, on the other hand, are methods employed by the less powerful to engage with strategic structures (The Practice of Everyday Life 94). How everyday citizens interact in the streets below the structures, however, cannot completely be controlled by these strategies. How users interact with art, networks, games, and culture can be distinguished in a similar fashion, although de Certeau notes that intended outcomes are impossible and irrelevant. Wark and Galloway's work on videogames is concerned with strategies more than with tactics. As a result, they tend towards analyzing the sources and structures of power as opposed to the users and uses of games.

In their writings about allegorithmic gaming, Galloway and Wark present effective materialist criticisms of mass culture as a strategic object, and, despite their attention to the details of power, gesture towards presently undefined tactics. One passage in particular from Wark's Gamer Theory indicates his hope for videogames: "Play may be unthinkable, but it nevertheless has a history, and that history traverses both cultural forms and the historical form of being itself. To approach it, to think this unthinkable category of play, is to play in and against language. Gamer theory calls for concepts that make the now rather familiar world of the digital game strange again" (224). Wark is hopeful and curious about a liberated gamespace, and invokes the Situationists as an example of a potential methodology for active game consumption. 
What this play looks like or how it is enacted is never quite clear from Wark's writing in Gamer Theory, but it is clear, much as it is in Galloway's introduction to Gaming, that they believe consumer tactics have liberating potential. A problem within the code does not, after all, indicate a problem within the social sphere.

Recettear and Papers, Please demonstrate potential for developer tactics to provide spaces of play free from co-opted labour and trade. According to Wark, when work and play combine, the former tends to co-opt and exert power over the latter. These games achieve a critical dystopian hope by reversing this process and recontextualizing work as a form of play. Tom Moylan and Raffaella Baccolini summarize critical dystopias as works that identify not only the symptoms of social unrest but also the causes, and are anxious and nervous about social conditions (Dark Horizons 4). Additionally, texts are considered critical dystopias when "the contemporary historical moment is interrogated by critical positions that necessarily work within a dystopian structure of feeling" (4). Elsewhere, Moylan elaborates that the critical dystopia "adopts a militant stance that is informed and empowered by a utopian horizon that appears in the text--or at least shimmers beyond its pages." (Scraps of the Untainted Sky 196). The diegetic worlds and narratives in these games is almost entirely dystopian, but the game play itself is made hopeful by reimaging work and laborious activity as a form of satirical play. By becoming commonplace, play is no longer play, and so we are faced with increasingly less interesting games. Popular game play focuses on dualisms which pit self against others, winning and losing, and encourage individual progress at the expense of communal growth. Recettear and Papers, Please, acknowledge, reject, and undermine these essentialisms. While Recettear satirizes market economics which praise the 
individual at the expense of community, Papers, Please highlights stagnant, exploitative, and dystopian play patterns that are normalized in many videogames. Both of these indie games are concerned with the growing routinization in games that results in non-play, and as a result attempt to resituate play within routinization.

\subsection{Trucking Along in Papers, Please}

Papers, Please is a game that attempts to antagonize its player base in various ways. The game takes place in a fictional dystopian Soviet-bloc country called Arstotzka sometime in the 1980s. The player acts as an immigration officer working to support his poverty-stricken family. A player's daily routine involves processing incoming travellers or returning citizens to the nation. The game play consists largely of standing at a desk and assessing whether or not passports or other documentation is properly supported to cross the border. The desk contains a rulebook with various visual cues for determining the integrity of documents provided by travellers. Players are rewarded 5 credits for each NPC processed through at the checkpoint. However, if a player mistakenly approves an NPC for travel to Arstotzka, either through forged or insufficient documents, they do not receive payment and are issued a citation. After a third citation the player is fined 5 credits for each mistake. The gamification of this bureaucratic process is accomplished by setting a time limit on the player's workday. A clock ticks throughout the workday, thereby limiting the attention players have for increasingly complex tasks. Players are asked to work, but also to work quickly, cross-referencing and double-checking documentation. Because payment is structured as piece work for each processed 
applicant, the longer players take processing each attempted entrant into the country the less money they will be paid per day. At the end of each day the game tracks how much money the player has made as well as costs such as rent, food, and medicine for an ailing and suffering family. The game intentionally frustrates players' abilities to process information quickly by adding new and complex rule structures for admitting travellers.

Each day begins with a headline from the national paper The Truth of Arstotzka, ${ }^{30}$ and a new rule for managing the checkpoint. For example, the first day begins with a rulebook that states only citizens of Arstotzka may cross the border upon re-entry home. The next day, however, the borders are opened to foreigners as well as citizens of Arstotzka. On the second day a terrorist detonates an explosion which kills a guard and ends the day early. Following this event, increasingly complex bureaucratic methods are employed to monitor the traffic at the border, including passports, entry tickets, ID cards, work and travel visas, and other supporting documents. Eventually, the player is also required to screen, interview, and subsequently detain suspicious persons. It becomes increasingly difficult to average even a dozen applicants per day, thereby limiting the amount of income. Additionally, emergency costs caused by illness or other issues suffered by the protagonist's family increase the pressure on the player to process migrants quickly.

Players who wish to be successful at the game must memorize neighbouring countries and their capitals, districts in Arstotzka, and other specific details because referring to the provided handbook for each possible migrant is too time consuming. Fake passports can range from obvious forgeries to almost identical replications. Date of 
birth, passport expiration, sex, issuing city, nation of origin, and photo must be verified on passports and other documents. For example, one fake passport a player encounters appears entirely legitimate except the issuing city is for the wrong country. Eventually, the player is contacted by the Order of the EZIC Star (EZIC), a revolutionary group who offers the player a bribe for various people to be let through without proper documentation. How the player manages these requests, as well as their money and other resources, results in one of twenty possible endings. Sometimes the player confounds an attempted coup by EZIC, while other times the player fills a key role in a successful revolution. Other times, the immigration officer's family simply fails to make enough money and dies. Or the immigration inspector is arrested for incompetency and sent to a GULAG. The endings are determined by the stressful banality that comprises each workday.

No matter the ending, the game requires players to comply with regulatory play patterns. As the border separating Arstotzka from other nations becomes increasingly porous, so too do the boundaries between play and labour. As the game progresses, it comes to resemble the real-world gamespace that Wark theorizes: the game is imperfect and difficult, and increasingly and varyingly difficult decisions and tasks arise that have no clear correct answer or decision tree. The game play is intentionally claustrophobic, thereby invoking a feeling of dread akin to the economic anxiety some players experience in gamespace. The game intentionally makes it difficult and unfair to support and provide for the player's family or for the player to win in any traditional sense. Mastering the allegorithm of the game requires players to internalize the rules of governance, nation, and bureaucracy. This game does not champion the individual 
performance and success that Wark describes as a property of other videogames. It does not contain the level playing field that other games provide players. Instead, the world of Papers, Please is a reflection of the real-word, what Wark refers to as gamespace. Lucas Pope, the game's designer, describes the game in a similar fashion: "my experiences with immigration led me to think there were interesting mechanics in the process. ... For me, having the rigid structure of a bureaucratic system makes designing the mechanics easier" ("Interview" n. pag.). Indie game designers, as noted earlier in this chapter, have keenly recognized videogames a site of contention between work and play.

The bleak setting of Arstotzka is not only a historical representation of a Sovietbloc nation, but an interpretation of our contemporary association of work and play. Players reiterate loops until their immigration officer reaches a morally ambiguous ending or is arrested, shot, or exiled along the way. Yet, the game itself is enjoyable. Bureaucratic repetition, it turns out, can be a satisfying puzzle and the decision to aid EZIC or let a desperate migrant through without proper documentation is exhilarating. The dystopian narrative that frames these game play decisions acknowledges the association between play and work. Rob Parker contends that Papers, Please explores "the fissures between a player's expertise and how that articulates itself through amoral or asocial behaviours ("The Art of Papers, Please" n. pag.). In order to unlock "Endless mode," for example, players must become a paragon of punitive bureaucracy by detaining suspects and reporting EZIC. To unlock endless mode, Parker notes, players must sublimate any heroic impulses and ignore the NPCs that ask for help. They must 'master' the game by being unsympathetic, by privileging game processes over their humanity.... Be a good, unthinking and obedient $\operatorname{cog}$ within the 
game's machinations and Papers, Please will reward you for it. It is as potent an indictment of game mastery as I have encountered, and 2013 has seen its share of AAA titles that have tried to complicate the heroic narrative tropes that accompany mechanical mastery or otherwise critique the affective inhumanity of procedural mastery - often problematically, and rarely with resounding success.

Here, Parker notes the desire and ability for indie game designers to subvert typical game design elements that praise essentialism. In addition to his critique of triple A titles, Parker praises the power of indie games to provide a satirical space for re-evaluating our contemporary forms of play. Capital's attempt to combine work and play, Wark reminds us, often results in work co-opting the latter. Papers, Please serves to remind us of this fact as well, but also provides an aesthetic reimagining of work as a tactical form of play. The variety of endings - potential revolution, arrest, escape, or exile - all indicate the possibility of change. Papers, Please acknowledges the association of play and work, yet, in critical dystopian fashion, gestures towards change or hope on the horizon.

\subsection{Bartering With Evil in Recettear}

Unlike the political despondency and depression that is central to satire in Papers, Please, Recettear's setting, characters, and world are exuberant and lively in the face of a narrative about exploitation. Recettear, unlike Papers, Please, is a transnational indie game that was localized to Western audiences from Japan by Carpe Fulgur studios. ${ }^{31}$

31

Localization is the process by which video games are converted, transformed, translated, and restructured to be sold in culturally, economically, or geographically distinct regions from which they were built. 
Mia Consalvo explains that Recettear reached indie markets in the West via "Westernbased anime and game fan sites such as RPGamer.com and Silicon Era, releasing regular news about the project and giving in depth interviews about the game's status and details about the localization process" (Atari to Zelda 144). Miguel Merino notes that, despite any particular game's status as indie, globalisation has increased demand for videogame translation ("On the Translation of Video Games" n. pag.). Generally, videogames are localized to appeal to the cultural tastes and desires of the intended consumers. Merino explains that the process of localization

will allow for transformations that will adapt the product to the local taste and expectations of the territory of release. The game does not have a traditional author, like a film director or a writer, but a 'shared authorship' because the only relevant issue is the product itself and how it woos the users by adapting to them. Therefore, everything in a video game is open to particular changes for specific territories, if it would mean a potential increase in sales. In other words, games may play and look different depending on the country where we purchase them. Merino differentiates video games from an artefact (a Picasso painting, for example) or a product (a car) because of their personalized appeal to specific audiences or cultures. In Recettear's case, the localization involved much less shared authorship than Merino and Consalvo's passage might indicate. In an interview on Gamasutra, Andrew Dice, one of two people who work at Carpe Fulgur, explains the minor alterations they made to the game: "It was a little bit weird when you'd have Recette and Tear talking about the poverty of their food, and then they'd be talking about rice and tofu and they were surrounded by very obviously French and Western buildings" ("Dice Talks Recettear, 
Indie Charm" n. pag.). The changes address food and setting, and the occasional phrasing, but the overall narrative remains largely unchanged.

Across geographic and cultural boundaries, indie games are concerned with the relationship between capitalism and gaming. The appeal of Recettear among Western and Japanese audiences reveals a common, global concern about the place of play, work, and exchange in contemporary gaming. ${ }^{32}$ Recettear is a meditative and satirical RPG that undermines the neoliberal produced claim to an autonomous and economically productive individual by invoking spectres of debt, loss, and servitude. Its themes and concerns, along with its market sales, are indicative of video games' involvement and collaboration with global capitalism.

Recette Lemongrass, the protagonist of the game, is a young girl whose father (Recet) has left to become an adventurer in pursuit of fame and fortune. Unfortunately, Recette's father perished in pursuit of his goals. In order to finance his exploits, Recette's father took a loan from a company and left his estate to her in his absence. Tear, a fairy who works for the collections portion of this company, appears at the beginning of the game to inform Recette that her father's estate will be confiscated unless she is able to repay his debt. Recette jokes that she will have to sell her organs to repay the debt, but Tear explains that the company would "never do something so cruel," and instead Tear will help turn her home into an item shop. With a narrative set, Recette begins her adventure of an indebted servant.

Like Papers, Please, time is measured in the form of debt repayment. Each day Recette sets up her shop and has a certain amount of time to acquire, organize, and sell (“Interview”).

Thinking they would sell only 10000 copies, Dice explains that they sold almost 300000 
items in her shop. The game is designed for the player (Recette) to pay off an instalment of her inherited debt at the end of each week. At the beginning of the game, Tear suggests Recette turn her family home into a place of business. The invasion of work into personal and play time is underscored from the very outset of the game. As if to highlight this point, Recette's room has a toy mushroom in the corner from the Mario videogame series. ${ }^{33}$ Where once treasures and items lurked for the singular adventurer in pursuit of defeating evil they were now commodities bought and sold. Recettear simultaneously recalls and troubles an era of RGPs that contained fairies, monsters, dragons, town halls, and high fantasy settings in which a single individual fought against another singular evil force.

Yet, Recettear understands that, unlike the fairy tale about a singular evil monster, evil is systemic. Writing about the use of the term evil, Imre Szeman explains that "the political operation of the fairy tale is thus to bring about a future in which evil is no longer part of the equation, having been challenged and exorcised by those interested in producing utopia out of the present's dystopia, its evil" ("Google and Evil as a Political Category" 129). The evil in Recettear is an alternative to the horrid denizens of dungeons associated with RGPs. In this game evil is the collusion of finance and game play. Szeman is concerned with Google's organization of knowledge through algorithmic functions he mistakes, yet his point about capitalism is relevant and poignant. Szeman attempts to introduce "an idea of systemic evil—an evil that is the outcome not of a singular extreme case, but of a specific political configuration of which an account bonus life, size, speed, and various other abilities and benefits across the dozens of games in the series. In the original platform games, they would be treasures found in a box or special place as Mario moved across a level. 
can be given and a judgement made" (131). The argument of liberal capitalism, Szeman explains, is that "economic evil, such as it is, occurs only at the extremes of capitalism; everywhere else and in all other cases, things are fine" (130-1). While the extreme case happens to Recette - that her father perishes and a bank comes after her, a child, for money - we see evil in the form of trade and play that take place in the game. In fact, at one point Recette jokes that she will have to sell her organs in order to repay the massive debt she owes to Tear's company. Tear explains that never something so cruel would be used and she would "help her instead." This, of course, is the systemic method of ensuring Recette not only pays but replicates the same structures that helped to enslave her.

There are a variety of practices in the game that mirror contemporary and mercantile methods of trading and exchange. The first stop for Recette and Tear on their adventure is the merchant's guild, where the master explains that all standard items are distrusted through the guild: "since the prices on items can vary from store to store, it's hard to do business if there isn't a base price between them, right?" "Capitalism, ho!" is Recette's common refrain to these sorts of interactions, to which Tear once responds "Well, Adam Smith would be proud of us, at any rate." In order to acquire items for their shop, Recette and Tear recruit an adventurer by funding his sword and shield and taking a large percentage of his loot. Recette and Tear begin a gold farming operation in which they pay a wage to a labouring adventurer. The two then sell these items to people who are not willing to adventure out into dungeons and wilderness to acquire these items. Having established themselves with the guild and a network of questing adventurers to exploit for additional profit, Recette and Tear finally embody their portmanteau. When 
Recette's debt is finally paid off, the game, like Papers, Please, enters "endless mode," where the debt is gone but the player can continue to build a stock, sell items, and acquire wealth. The narrative satirizes exactly what Szeman describes as capitalism's “denial of the structural, systemic injustice - the injustice that accumulates by a thousand cuts through otherwise seemingly banal practices and beliefs" (134).

That "Endless mode" is a final game state for both Recettear and Papers, Please indicates the iterative loops of capital these games critique. Both games are exceptionally critical of capitalism's continued efforts to appropriate spaces of play through repetition and non-play. By referencing these appropriations, both games also allude to the endless cycles of capitalist expansion defined by Autonomists discussed earlier. Yet, at the same time, endless mode offers a form of exfoliation that Harpold finds liberating for game players, as a sort of active consumption that frees users from purchasing and requiring new products and consoles. The games can increase difficulty levels in this mode and offer new puzzles and challenges for players. Providing users with endless modes and replay value (particularly the case in Papers, Please with its 20 possible endings) reflects the design principles behind the auction house and money systems in MMOs like World of Warcraft. The difference between these games and WoW, however, is that they are offline, removed from participating in a larger economy, and therefore instead can offer alternative commentary on the practices of trade and exchange. 


\section{Conclusion}

This research is particular to three specific trade interfaces, two single-player game texts, and one novel. Because it is specific to particular advancements across these objects, it is important to recognize the limitations of constructing a broad description regarding online trade practices in general. Nevertheless, the trade advancements in World of Warcraft and the Steam Market are emblematic, as Reamde, Recettear, and Papers, Please indicate, of extensive concern regarding labour, trade, and capitalism in contemporary gaming. Moreover, this work provides important historic assessments of early MMO trade practices in light of their developments over twenty years.

Furthermore, this dissertation constructs a specifically Marxist narrative regarding the rise of automation in trading. While this particular methodology may invite certain

criticism from other schools of thought, the information and technical research provided 
in the literature review represent a sound collection of synthesized research on the broad topic of online trading. Moreover, this dissertation builds upon a fundamental Autonomist Marxist research by presenting video game players as socialised, active players who maintain agency within a hostile and exploitative economic market. With advances in online trade and consumption, this research represents an important intervention in the growing field of video game studies. In particular, it demonstrates the ideological qualities central to contemporary trade practices and their associated user interfaces. In so doing, this dissertation critiques the increasingly non-gamic elements that constitute contemporary game design.

Moving forward, there are a few avenues of research I would like to expand upon. In the introduction, I spoke of the structures outside of the game that stood as a bulwark against player agency. Perhaps in future research it might be useful to identify different types of agency and investigate the term more thoroughly. For example, it might be helpful to separate player agency with regards to rule structures, game play, role play, mechanics, social life, and secondary markets. Players may have agency in some facets of any particular game and not others. Moreover, there are active players or agents in games that see them as productive sites of labour and value creation. Players are diverse in their interest and decisions, but they are competing as well with the agency and interests of capitalism and its proponents.

One avenue for further research would involve ethnographic work surrounding The Realm. Acquiring information and research on this and other early MMOs can prove quite difficult, and contributing to the amount of information available regarding these objects would provide a useful source of academic material for future research. 
Additionally, similar work can be done on World of Warcraft's Auction House. One method of continuing research on the Auction House would involve a closer inspection of the various patch notes and changes that have been made to it over the years. While I mention one patch change in particular, there is an enormous amount of potential in reading through patch notes to understand the intricacies of trade interfaces from a designer's perspective. Another method available for studying both the Steam Market and WoW's Auction House would be to employ some form of computational sentiment analysis on publically available forums. Moreover, it might be useful to integrate more quantitative economic analysis, similar to Castranova's work, regarding the efficacy of trade interfaces.

Nevertheless, this dissertation demonstrates the increasingly abstract nature of play in its relation to labour, particularly as it concerns trade interfaces. By doing so, this dissertation shows how ostensibly pedestrian game design elements influence practical behaviours among players. Players, as Sicart notes, must have the ability to influence or make active, informed decisions about their game play in order for a design to be considered ethical. As a result, this dissertation is able to make an ethical critique about the state of trade interfaces in three distinct online markets. Additionally, this dissertation demonstrates a broader cultural concern regarding virtual trade among nonmultiplayer texts, such as Recettear, Papers, Please, and Reamde.

Ultimately, this dissertation demonstrates the increasing reliance on efficiency concerning digital trade practices. By prioritizing efficiency, video game trading has become a less playful act, according to Suits's definition of a game provided in the Introduction. The story of interface development is one in which players become figures 
and statistics in an ever-expanding market. This research demonstrates that interfaces progressively trend towards optimizing economic interaction between players, and thereby disregard potential social, cultural, and playful interactions among players. Perhaps most importantly, this dissertation moves beyond neoclassical economic constructions that fail to assess contemporary global capitalism. Instead, this dissertation views economic interaction in relation to its global position and circulation of wealth, control, and capital. Finally, it accepts that assessing any single currency, market, or economy in a vacuum is not possible under contemporary global capitalism, and therefore seeks to describe the general trends and behaviours that constitute video game trading. 


\section{Works Cited}

Aarseth, Espen. Cybertext: Perspectives on Ergodic Literature. Baltimore: John Hopkins UP, 1997. Print.

"The Absurdity of the \#FreeSwag Movement." YouTube, uploaded by Richard Lewis, 24 April 2017. www.youtube.com/watch?v=pq3cDfVHJnE

Alty, J.L, R.P. Knott, B. Anderson, and M. Smyth. “A Framework for Engineering Metaphor at the User Interface.” Interacting With Computers 13 (2000). 301-322. Print.

Ashcraft, Brian. "Going Indie: Fez Creator Phil Fish's Moment of Clarity. Kotaku. 20 April 2009. Web. 5 May 2017. kotaku.com/5219689/going-indie-fez-creatorphil-fishs-moment-of-clarity

"Auction House Guide - Making A WoW Fortune." WoWHead. Web. 2 May 2017. www.wowhead.com/guide=1076/auction-house-guide-making-a-wow-fortune.

"The Awp Nerf." YouTube, uploaded by Thooorin, 6 April 2015. www.youtube.com/watch? $\mathrm{v}=\mathrm{rsFnJYJ} 2 \mathrm{buU}$

Baccolini, Raffala and Tom Moylan. Dark Horizons. New York: Routledge, 2003. Print. Blizzard Entertainment. Diablo III. Blizzard, 2012. Windows.

---. World of Warcraft. Blizzard, 2004. Windows.

Bogost, Ian. "Gamification is Bullshit." Bogost.com. 8 Aug 2011. Web. 2 Feb 2014. bogost.com/writing/blog/gamification_is_bullshit/

---. Persuasive Games: The Expressive Power of Videogames. Cambridge: MIT, 2007. Print. 
Boluk, Stephanie. "Steam Works." Current Projects. Web. 5 April 2016. stephanieboluk.com/

Bourdieu, Pierre. The Field of Cultural Production: Essays on Art and Literature. Ed. Randal Johnson. New York: Columbia UP, 1993. Print.

Bowman, Mitch. "The Hidden World of Steam Trading." Polygon. 22 May 2014. Web. 9 April 2017. www.polygon.com/features/2014/5/22/5590070/steam-valve-itemtrading.

Brousseau, Eric and Jean-Michel Glachant. New Institutional Economics: A Guidebook. Cambridge: Cambridge UP, 2009. Print.

Bryan, Dick and Michael Rafferty. Capitalism with Derivatives: The Political Economy of Financial Derivatives, Capital, and Class. London: Palgrave, 2005. Print.

Burden, Michael and Sean Gouglas. "The Algorithmic Experience: Portal as Art." Game Studies 12.2 (2012). Web. 7 Feb 2014. gamestudies.org/1202/articles/the_algorithmic_experience

Burdick, Anne, Joanne Drucker, Peter Lunenfeld, Todd Presner, and Jeffrey Schnapp. Digital Humanities. Cambridge: MIT Press, 2012. Print.

Calleja, Gordon. In-Game: From Immersion to Incorporation. Cambridge: MIT, 2011. Print.

Castranova, Edward. Exodus to the Virtual World. New York: Palgrave, 2008. Print. ---. "On Virtual Economies." Game Studies 3.2 (2003). Web. 9 Sept 2017. http://www.gamestudies.org/0302/castronova/ --- "The Price of 'Man' and 'Woman"' ---. Synthetic Worlds: The Business and Culture of Online Games. Chicago: U of Chicago 
P, 2005. Print.

CCP Games. EVE Online. Atari and Valve, 2003. Windows and Linux.

Clark, Andy. Natural Born Cyborgs. Oxford: Oxford UP, 2004. Print

Consalvo, Mia. Atari to Zelda: Japan's Videogames in Global Contexts. Cambridge: MIT Press, 2016. Print.

"DaZeD about Valve." YouTube, uploaded by Seon Highlights \& Vods, 24 July 2016. www.youtube.com/watch? $\mathrm{v}=\mathrm{dm} 3 \mathrm{kjN} 7 \mathrm{uZZA}$

de Certeau, Michel. The Practice of Everyday Life. Berkley: U of California P, 1988. Print.

Dean, Jodie. Blog Theory: Feedback and Capture in the Circuits of Drive. London: Polity, 2010. Print.

DeLanda, Manuel. New Philosophy of Society: Assemblage Theory and Social Complexity. New York: Continuum, 2006. Print.

Dibbell, Julian. "The Life of a Chinese Gold Farmer." New York Times. 17 June 2007. Web. 13 Oct 2017. www.nytimes.com/2007/06/17/magazine/17lootfarmerst.html.

Dice, Andrew. Interviewed by Kyle Orland. Gamasutra. 9 Sept 2010. Web. 5 April 2017. www.gamasutra.com/view/news/121067/Interview_Carpe_Fulgurs_Dice_Talks_ Recettear_Indie_Charm.php

Drobak, John. "Introduction: Law and New Institutional Economics." Journal of Law and Policy. Vol. 26 (2008). Web. 9 April 2017. openscholarship.wustl.edu/law_journal_law_policy/vol26/iss1/2/ 
Dyer-Witherford, Nick. Cyber-Marx: Cycles and Circuits of Struggle in High Technology Capitalism. Chicago: U of Illinois P, 1999. Print.

Dyer-Witherford, Nick and Greig de Peuter. Games of Empire: Global Capitalism and Video Games. Minneapolis: U of Minnesota P, 2009. Print.

Fuchs, Christian. Digital Labour and Karl Marx. New York: Routledge, 2014. Print.

Fuchs, Christian and Sebastian Sevignani. "What is Digital Labour?" Communication, Capitalism, and Critique 11.2 (2013): 237-293. Print.

Gadamer, Hans-Georg. Gadamer on Celan: "Who am I and who are You?" and Other Essays. New York: U of New York P, 1997. Print.

Galloway, Alexander. Gaming: Essays on Algorithmic Culture. Minneapolis: U of Minnesota P, 2006. Print.

---. The Interface Effect. Malden: Polity, 2012. Print.

---. "The Poverty of Philosophy: Realism and Post-Fordism." Critical Inquiry 39.2 (2013): 347-366. Print.

Grundhauser, Eric. "It's a Mystery Who's Running One of the Oldest Multi-Player Online Fantasy Games." AtlasObscura.com. 5 Aug 2016. Web. 9 Sept 2016. www.atlasobscura.com/articles/its-a-mystery-whos-running-one-of-the-oldestmultiplayer-online-fantasy-games.

Hall, Charlie. "Pro CS:GO players issue open letter." Polygon. 22 Dec 2016. Web. 15 Jan 2017. www.polygon.com/2016/12/22/14057332/pea-professional-esportsassociation-cs-go-players-letter

Hardt, Michael and Antonio Negri. Empire. Oxford: Harvard UP, 2000. Print.

---. Multitude: War and Democracy in the Age of Empire. New York: Penguin, 2004. 
Print.

Harpold, Terry. Ex-foliations: Reading Machines and the Upgrade Path. Minneapolis: U of Minnesota P, 2008. Print.

Heinrich, Michael. An Introduction to the Three Volumes of Karl Marx's Capital. New York: MR Press, 2004. Print.

Hellstrom, Christopher. Alpha Geek: Neal Stephenson, the Emerging Third Culture, and the Significance of Science Fiction. 2015. St. John's U, PhD dissertation. searchproquestom.proxy.library.carleton.ca/docview/1708335623/D2F87335C3A64422 $\mathrm{PQ} / 15$ ?accountid=9894.

Herman, Nancy J. Deviance. Lanham: Rowman and Littlefield, 1995. Print.

Horkheimer, Max, and Theodor W. Adorno. Dialectic of Enlightenment. Ed. Gunzelin Schmid Noerr. Redwood City: Standford UP, 2002. Print.

Huizinga, Johan. "Nature and Significance of Play." The Game Design Reader, Eds

Katie Salen and Eric Zimmerman. Cambridge: MIT Press, 2006. 96-119. Print.

Johnson, Erik. "In-Game Item Trading Update." SteamPowered. 13 July 2016. Web. 5 April 2017. http://store.steampowered.com/news/22883/

Johnson, Soren. “Theme is not Meaning." Steinkuehler, Squire, and Barab 32-39.

Jones, Steven E. The Meaning of Video Games. New York: Routledge, 2011. Print.

Jupin, Tanner. Gamic Fiction: the Intermediation of Literature and Games. 2014. University of California, PhD dissertation.

Juul, Jesper. "The Game, the Player, the World: Looking for a Heart of Gameness." Level Up: Digital Games Research Conference Proceedings. Eds Marinka Copier and Joost Raessens. Utrecht: Utrecht University,2003. Web. 9 April 2017. 30-35. 
www.jesperjuul.net/text/gameplayerworld/

---. "Zero-Player Games." Philosophy of Computer Games Conference. 2012. Web. 9

May 2017. www.jesperjuul.net/text/zeroplayergames/

Keeler, Mary A. and Susan M. Denning. "The Challenge of Interface Design for Communication Theory: From Interaction Metaphor to Contexts of Discovery." Interacting With Computers 3.3 (1991): 283-301. Print.

"Kings Quest V." Wikipedia: The Free Encyclopedia. Wikimedia Foundation, Inc. 28 Aug 2017. Web. 11 Sept 2017. en.wikipedia.org/wiki/King\%27s_Quest_V.

Lastowka, Greg. "Planes of Power: EverQuest as Text, Game and Community." Game Studies 9.1 (2009). Web. 9 April 2014. gamestudies.org/0901/articles/lastowka

Lehdonvirta, Vili. Virtual Consumption. 2009. Turku School of Economics, PhD dissertation.

Lieberherr, Eva. "Policy Relevance of New Institutional Economics? Assessing Efficiency, Legitimacy, and Effectiveness. " Discussion paper series on the Coherence between institutions and technologies in infrastructures. Print.

Little, Daniel. "Marxism and Method." Web. 20 April 2016. wwwpersonal.umd.umich.edu/ delittle/resources/Marxism\%20and\%20method.pdf

Lynn, Casey. "RPG Nostalgia: The Realm." GAS. 17 Nov 2008. Web. 9 April 2017. www.geeksaresexy.net/2008/11/17/rpg-nostalgia-the-realm/.

Malaby, Thomas. "Culture versus Architecture.” Steinkuehler, Squire, and Barab 229243.

---. Making Virtual Worlds: Linden Lab and Second Life. Ithaca: Cornell UP, 2009. Print. 
Marx, Karl. Grundrisse. London: Penguin, 1993. Print.

Mauss, Marcel. The Gift: Forms and Functions of Exchange in Archaic Societies. London: Routledge, 2002. Print.

Mosco, Vincent. Digital Sublime: Myth, Power, and Cyberspace. Cambridge: MIT Press, 2004. Print.

Moylan, Thomas. Scraps of the Untainted Sky: Science Fiction, Utopia, Dystopia. New York: Avalon, 2000. Print.

Nakamura, Lisa. Cybertypes: Race, Ethnicity, and Identity on the Internet. New York: Routledge, 2001. Print.

Nardi, Bonnie. My Life as a Night Elf Priest. Ann Abror: U of Michigan P, 2010. Print. Nichols, Stephen. Interviewed by Justin Olivetti. The Game Archaeologist. 17 Nov 2012. Web. 9 April 2017. www.engadget.com/2012/11/17/the-gamearchaeologist-steve-nichols-debriefs-the-realm-online/.

North, Douglas. "Institutions." Journal of Economic Perspectives 5.1 (1991): 97-112. Print.

Novy-Williams, Eben and Joshua Brustein. "Virtual Weapons are Turning Teens into Serious Gamblers." Bloomberg. 20 April 2016. Web. 5 May 2016. www.bloomberg.com/features/2016-virtual-guns-counterstrike-gambling/

Parker, Rob. "The Art of Papers, Please." First Person Scholar. 23 Oct 2013. Web. 5 Feb 2015. www.firstpersonscholar.com/the-art-of-papers-please/

"Patch Notes." BattleNet. 28 March 2017. Web. 6 June 2017. us.battle.net/wow/en/game/patch-notes/.

Pearce, Celia. Communities of Play: Emergent Cultures in Multiplayer Games and 
Virtual Worlds. Bogart: MIT, 2009. Print.

---. "Narrative Environments From Disneyland to World of Warcraft."Space, Time, Play: Computer Games, Architecture and Urbanism: The Next Level. Eds. Friedrich

von Borries, Steffan P. Walz, and Matteas Bottger. Basel: Birkhauser, 2007. 200-205. Print.

Pedercini, Paolo. "Videogames and the Spirit of Capitalism." Molleindustria. 14 Feb 2014. Web. 2024 Nov 2015. www.molleindustria.org/blog/videogames-andthe- spirit-of-capitalism/

"The Realm Online." GiantBomb Game Wiki. 8 Jan 2015. Web. 9 April 2017. www.giantbomb.com/the-realm-online/3030-8999/.

Rothbard, Murray. An Austrian Perspective on the History of Economic Thought Before Adam Smith. Cheltenham: Edward Elgar, 1995. Print.

Screpanti, Ernesto and Stefano Zamagni. An Outline of the History of Economic Thought. 2nd ed. Trans. David Field and Lynn Kirby. Oxford: Oxford UP, 2005. Print. Seriosity Attent. Seriosity, Inc, 2010. Windows.

Sicart, Miguel. The Ethics of Computer Games. Cambridge: MIT Press, 2009. Print. Sierra Online. The Realm Online. Sierra Online, 1996. Windows.

Simmel, Georg. "The Metropolis and Mental Life." The Blackwell City Reader. Eds Gary Bridge and Sophie Watson. Oxford: Wiley-Blackwell, 2002. 11-19. Print.

Simon, Bart. "Indie, Eh? Some kind of Game Studies." Loading... 7.11 (2013): 1-7. Print.

"Skin Gambling." Wikipedia: The Free Encyclopedia. Wikimedia Foundation, Inc. 10 Oct 2017. Web. 12 Oct 2017. en.wikipedia.org/wiki/Skin_gambling 
Smith, Adam. The Wealth of Nations. Edited by Charles J. Bullock. New York: Cosimo, 2007. Print

Spithoven, Antoon, John Groenewegen, and Anenette Van den Berg. Institutional Economics: An Introduction. London: Palgrave, 2010. Print.

Squire, Kurt. "Designed Cultures." Steinkuehler, Squire, and Barab 10-31.

Steinkuehler, Constance, Kurt Squire, and Sasha Barab, eds. Games, Learning, and Society: Learning and Meaning in the Digital Age. Cambridge: Cambridge UP, 2012. Print.

Suits, Bernard. The Grasshopper. Peterborough: Broadview, 2014. Print.

Szeman, Imre. “'Do No Evil': Google and Evil as a Political Category." Topia 18 (2007): 127-134). Print.

Tally, Robert T. Jr. "Meta-Capital: Culture and Financial Derivatives." Works and Days 30.1 (2012): 231-255. Print.

Terranova, Tiziana. Network Culture: Politics for the Information Age. London: Pluto, 2004. Print.

"Trading Guides!" SandyClaws' Realm Site. 23 Dec 2012. Web. 9 April 2017. realm.holtgard.com/index.html.

"Trading and Market Restrictions." Steam. Web. 18 Feb 2016. support.steampowered.com/kb_article.php?ref=1047-EDFM-2932.

Tringham, Neal. Science Fiction Video Games. Boca Raton: CRC Press, 2015. Print. Turkle, Sherry. Life On the Screen. New York: Simon and Schuster, 1995. Print. Varoufakis, Yanis. "Arbitrage and Equilibrium in the Team Fortress 2 Economy." Valve. $\quad 22$ June 2012. Web. 10 April 2015. 
blogs.valvesoftware.com/economics/arbitrage-and-equilibrium-in-the-teamfortress-2-economy/

---. Interviewed by Doug Henwood. 11 July 2012. Web. 10 Feb 2015. www.yanisvaroufakis.eu/2012/07/11/interviewed-by-doug-henwood-on-my-newposition-as-economist-in-residence-at-valve-software/

---. "To Truck, Barter, and Exchange." Valve. 1 July 2012. Web. 10 April 2015. blogs.valvesoftware.com/economics/to-truck-barter-and-exchange-on-the-natureof-valves-social-economies/

Vega, Sin. "The Art of the Deal: Trading Games Dont Understand Trade." RockPaperShotgun. 23 March 2016. Web. 9 April 2017. www.rockpapershotgun.com/2016/03/23/games-trading/

Wark, McKenzie. A Hacker Manifesto. Cambridge: Harvard UP, 2004. Print.

---. Gamer Theory. Cambridge: Harvard UP, 2007. Print.

Wending, Amy. Karl Marx on Technology and Alienation. London: Palgrave, 2009. Print.

Williams, Ian. “On Geek Culture.” Jacobin. 8 May 2013. Web. 20 July 2015. www.jacobinmag.com/2013/08/on-geek-culture.

Williamson, Oliver E. "The New Institutional Economics: Taking Stock, Looking Ahead." Journal of Economic Literature 38.3 (2000): 595-613. Print.

"Working the Auction House." WoWWiki. Web. 9 April 2017. wowwiki.wikia.com/wiki/Working_the_Auction_House.

Yee, Nick. The Daedalus Project. 4 Dec 2013. Web. 6 Sept 2017. www.nickyee.com/daedalus/archives. 
"Your Realm History." Community.TheRealm forum post. Sept 2016. Web. 20 April 2017. community.therealm.io/t/your-realm-history-its-heyday-poll/264/75

Zimmerman, Eric. "Jerked Around by the Magic Circle." Gamasutra. 7 Feb 2012. Web. 10 April 2017.www.gamasutra.com/view/feature/135063/jerked_around_by_the_ magic_circle_.php

Zimmerman, Eric and Katie Salen. Rules of Play. Cambridge: MIT Press, 2003. Print. 